\title{
RANDOM WALKS AMONG TIME INCREASING CONDUCTANCES: HEAT KERNEL ESTIMATES
}

\author{
AMIR DEMBO, RUOJUN HUANG, AND TIANYI ZHENG
}

\begin{abstract}
For any graph having a suitable uniform Poincaré inequality and volume growth regularity, we establish two-sided Gaussian transition density estimates and parabolic Harnack inequality, for constant speed continuous time random walks evolving via time varying, uniformly elliptic conductances, provided the vertex conductances (i.e. reversing measures), increase in time. Such transition density upper bounds apply for discrete time uniformly lazy walks, with the matching lower bounds holding once the parabolic Harnack inequality is proved.
\end{abstract}

\section{INTRODUCTION}

One of the most studied models for random walks in disordered media is the (random) conductance model, based on a locally finite, connected, non-oriented, graph $\mathbb{G}=(V, E)$ equipped with a (random) collection of symmetric strictly positive, edge conductances $\boldsymbol{\Pi}:=\{\pi(x, y)>0:(x, y) \in$ $E\}$ (for example, see $\mathrm{MB}, \mathrm{Kum}$ and references therein). We consider here random walks among non-random but time-varying, edge-wise Borel measurable, conductances $\boldsymbol{\Pi}_{t}$. In particular, taking $\boldsymbol{\Pi}_{t}=\boldsymbol{\Pi}_{[t]}$, the discrete time (simple) random walk (DTRW) $\left\{X_{n}\right\}$ on the corresponding sequence of weighted graphs $\boldsymbol{G}_{n}=\left(\mathbb{G}, \boldsymbol{\Pi}_{n}\right)$ is constructed as a time-in-homogeneous $V$-valued Markov chain, having for $t \in \mathbb{N}$ the transition probabilities

$$
K_{t}(x, y):=\frac{\pi_{t}(x, y)}{\pi_{t}(x)}, \quad(x, y) \in E, t \geq 0,
$$

each of which is reversible with respect to the vertex conductances

$$
\pi_{t}(x):=\sum_{\{y:(x, y) \in E\}} \pi_{t}(x, y) .
$$

Any such $\pi_{t}(\cdot)$ is a $\sigma$-finite measure on $V$ and we denote by $\mathcal{M}_{+}(V)$ those $\sigma$-finite measures which are bounded away from zero, namely functions $\mu: V \mapsto(0, \infty)$ with $\mu:=\inf _{x} \mu(x)>0$.

More generally, starting at $K_{n, n}=I$, any transition probabilities $\left\{K_{\ell}\right\}$ on $V$, induce the timeinhomogeneous transitions

$$
K_{k-1, n}(x, z):=\mathbb{P}\left(X_{n}=z \mid X_{k-1}=x\right)=\sum_{y \in V} K_{k}(x, y) K_{k, n}(y, z), k \leq n .
$$

Similarly, for $\left\{K_{t}\right\}$ of (1.1), the constant speed random walk (CSRW), is the $V$-valued stochastic process of RCLL sample path $t \mapsto Y_{t}$ that at the arrival times $\left\{T_{n}\right\}$ of an auxiliary unit rate Poisson

Date: December 4, 2018.

2010 Mathematics Subject Classification. Primary 60J35; Secondary 60J05, 60J25, 60J45.

Key words and phrases. Heat kernel estimates, parabolic Harnack inequality, time-dependent random walks, conductance models, stability.

This research was supported in part by NSF grant DMS-1613091. 
process, jumps to $y \neq x$ according to

$$
\mathbb{P}\left(Y_{T_{n}}=y \mid Y_{T_{n}^{-}}=x\right)=K_{T_{n}}(x, y), \quad(x, y) \in E, n \in \mathbb{N} .
$$

The CSRW is thus a time-inhomogeneous Markov process. Its transition probabilities $K_{s, t}(x, z)=$ $\mathbb{P}\left(Y_{t}=z \mid Y_{s}=x\right)$ satisfy the inhomogeneous semi-group property $K_{s, u} K_{u, t}=K_{s, t}$ for any $s<u<t$. Furthermore, such $\left\{K_{s, t}(x, z)\right\}$ solve with (initial) condition $u(t, x)=\mathbf{1}_{\{z=x\}}$, the backward equation

$$
u(s, x):=\mathbb{E}\left[u\left(t, Y_{t}\right) \mid Y_{s}=x\right]=e^{-(t-s)} u(t, x)+\int_{s}^{t} e^{-(\xi-s)} d \xi \sum_{y \in V} K_{\xi}(x, y) u(\xi, y),
$$

which upon setting $\left(\mathcal{L}_{s} f\right)(x):=\sum_{y \in V}(f(y)-f(x)) K_{s}(x, y)$, amounts to the distributional solution of $\partial_{-s} u=\mathcal{L}_{s} u(s, \cdot)($ see $(1.23))$.

Any conductance model, having $\boldsymbol{\Pi}$ independent of $t$, is a reversible, time-homogeneous network, of reversing measure $\{\pi(x): x \in V\}$. Time varying $\boldsymbol{\Pi}_{t}$ for which $\pi_{t}(x)=\pi(x)$ are independent of $t$, retain this reversibility (even though they form time-inhomogeneous transitions $K_{s, t}$ ). In contrast, as soon as $\left\{\pi_{t}(x): x \in V\right\}$ changes with time $(t)$, the dynamics associated with (1.3) or with (1.5) become genuinely non-reversible. Nevertheless, it has been suggested in ABGK] that some universality applies for the recurrence versus transience of such dynamics. Specifically, ABGK, Conj. 7.1] conjectures that if both conductance models corresponding to $\boldsymbol{G}_{0}$ and $\boldsymbol{G}_{\infty}$ are recurrent, or alternatively, both $\boldsymbol{G}_{0}$ and $\boldsymbol{G}_{\infty}$ are transient, then the same holds for the dynamic of non-decreasing $\left\{n \mapsto \boldsymbol{G}_{n}\right\}$, namely the DTRW evolving according to (1.3). Indeed, using flows to construct suitable sub or super-harmonic functions, such universality is established in ABGK, Sec. 5] when $\mathbb{G}=\mathbb{T}$ is a tree in the recurrent case, and when $\mathbb{G}=\mathbb{N}$ in the transient case, even allowing for conductances $\boldsymbol{\Pi}_{n}$ adapted to the path $\left\{X_{k}, k \leq n\right\}$. In contrast, [ABGK, Sec. 6] shows that such universality fails for randomly adapted, increasing in time, conductances on $\mathbb{G}=\mathbb{Z}^{2}$, whereas [ABGK, Ex. 3.5 and 3.6] demonstrates such failure in the non-adapted and non-monotone setting (even on the trivial tree $\mathbb{G}=\mathbb{Z}$ ).

The intuition behind ABGK, Conj. 7.1] owes to the equivalence between conductance models and electrical networks, yielding key comparisons such as Rayleigh monotonicity principle (due to which the random walk on any sub-graph $\mathbb{G}^{\prime}$ of a recurrent $\mathbb{G}$ must also be recurrent). Lacking such comparisons for the time-varying conductances of (1.1), we instead seek alternative analytic tools, such as, establishing the relevant (two-sided) Gaussian transition density estimate under certain geometric assumptions on the underlying graphs. That is, to show that for some $C$ finite, suitable measures $\mu_{s, t}(\cdot)$, all $x, y \in V$ and $t-s \geq d(x, y)$,

$$
\begin{array}{ll}
(\mathrm{GHKU}) & K_{s, t}(x, y) \leq \frac{C \mu_{s, t}(y)}{\pi_{s}(\mathbb{B}(y, \sqrt{t-s}))} \exp \left(-\frac{d(x, y)^{2}}{C(t-s)}\right), \\
(\mathrm{GHKL}) & K_{s, t}(x, y) \geq \frac{C^{-1} \mu_{s, t}(y)}{\pi_{s}(\mathbb{B}(y, \sqrt{t-s}))} \exp \left(-\frac{C d(x, y)^{2}}{t-s}\right),
\end{array}
$$

where $d(x, y)=d_{\mathbb{G}}(x, y)$ and $\mathbb{B}(y, r):=\{z \in V: d(y, z) \leq r\}$ denote the graph distance in $\mathbb{G}$ and the corresponding $\mathbb{G}$-ball, respectively. Compared to classical Gaussian heat kernel estimates (GHKE), the novel feature in (1.6) and (1.7) is the presence of the time dependent reference measures $\mu_{s, t}$. Indeed, a key difficulty for applying analytic methods to the time-varying conductance model is that the time-inhomogeneous transition probabilities $\left\{K_{s, t}\right\}$ do not admit any common invariant measure. While in principle one has the freedom to choose $\mu_{s, t}(\cdot)$, we shall take

$$
\mu_{s, t}(y):=\left(\pi_{s} K_{s, t}\right)(y)=\sum_{x \in V} \pi_{s}(x) K_{s, t}(x, y), \quad t \geq s \geq 0 .
$$


These measures play the role of invariant measures by satisfying the relation

$$
\mu_{s, t}(y)=\sum_{x \in V} \mu_{s, v}(x) K_{v, t}(x, y), \quad \forall s \leq v \leq t,
$$

with the GHKU and GHKL thus viewed as Gaussian heat kernel estimates of $\left\{K_{s, t}\right\}$ with respect to such evolving reference measure. We say that $\left\{\boldsymbol{G}_{t}\right\}$ satisfies the uniform volume doubling (VD) condition, if

$$
\sup _{t, r \geq 0} \sup _{x \in V}\left\{\frac{\pi_{t}(\mathbb{B}(x, 2 r))}{\pi_{t}(\mathbb{B}(x, r))}\right\} \leq C_{D}<\infty
$$

and further motivate our specific choice (1.8) by noting that for $\left\{\boldsymbol{G}_{t}\right\}$ satisfying such VD condition, if both (1.6) and (1.7) are to hold, then one must take for $\mu_{s, t}$ in the GHKU and GHKL, up to a universal constant, the measures from (1.8). (Indeed, for $t=s$ compare (1.6) and (1.7) at $x=y$, while for $t>s$ bound $\pi_{s} K_{s, t}$ below using (1.7) and the fact that $d(x, y)^{2} /(t-s) \leq 1$ on $\mathbb{B}(y, \sqrt{t-s})$, then rely on (1.9) at $r_{k}=2^{k} \sqrt{t-s}, k \geq L$, when bounding $\pi_{s} K_{s, t}$ above via (1.6), to deduce that necessarily $\left(\pi_{s} K_{s, t}\right) / \mu_{s, t} \in\left[c_{\star}^{-1}, c_{\star}\right]$ for some universal $\left.c_{\star}<\infty\right)$.

In this work we mainly focus on the case that for some $\pi_{0} \in \mathcal{M}_{+}(V)$, the reversing measures of $\left\{K_{t}\right\}$ form a pointwise non-decreasing sequence $t \mapsto \pi_{t}(x)$, of positive functions on $V$. It is a delicate issue that one must impose certain constraints on the measures $\pi_{t}$ : for if $t \mapsto \pi_{t}(x)$ are allowed to oscillate, then anomalous behavior may occur (cf. ABGK, HK, SZ3]). Our main result for CSRW is such two sided Gaussian estimates with respect to $\mu_{s, t}$, under uniform volume doubling and Poincaré inequalities. Necessary definitions are listed as follows.

Definition 1.1. We say that the uniform Poincaré inequality holds for $\pi_{t}$-reversible $K_{t}$, if

$$
\inf _{f_{\star} \in \mathbb{R}}\left\{\sum_{x \in \mathbb{B}\left(x_{0}, r\right)}\left|f(x)-f_{\star}\right|^{2} \pi_{t}(x)\right\} \leq \mathrm{C}_{\mathrm{P}} r^{2} \sum_{x, y \in \mathbb{B}\left(x_{0}, 2 r\right)}(f(x)-f(y))^{2} K_{t}(x, y) \pi_{t}(x),
$$

for some $\mathrm{C}_{\mathrm{P}}<\infty$ and all $f: V \rightarrow \mathbb{R}, x_{0} \in V, t, r \geq 0$.

- The uniform volume growth with $v(r)$ doubling applies for $\mathbb{G}=(V, E)$ and $\pi_{t}: V \rightarrow \mathbb{R}_{+}$, if for some $C_{v}<\infty$,

$$
\mathrm{C}_{\mathrm{v}}^{-1} \leq \frac{\pi_{t}(\mathbb{B}(x, r))}{v(r)} \leq \mathrm{C}_{\mathrm{v}}, \quad \forall x \in V, \quad \forall r, t \geq 0
$$

where $v(\cdot)$ is non-decreasing, $v(2 r) \leq \mathrm{C}_{\mathrm{v}} v(r)$ and $v(0)=v(1)=1$. In particular, then $\pi_{t}(x) \in$ $\left[\mathrm{C}_{\mathrm{v}}^{-1}, \mathrm{C}_{\mathrm{v}}\right]$ for all $t, x$ and the uniform vD condition holds (with $\mathrm{C}_{\mathrm{D}}=\mathrm{C}_{\mathrm{v}}^{3}$ in (1.9)).

- We call Markov kernels $\left\{K_{t}\right\}$ uniformly lazy and weighted graphs $\left\{\boldsymbol{G}_{t}\right\}$ uniformly elliptic, if respectively,

$$
\begin{aligned}
& \alpha_{l}:=\inf _{t} \inf _{x \in V}\left\{K_{t}(x, x)\right\}>0, \\
& \alpha_{e}:=\inf _{t} \inf _{(x, y) \in E}\left\{K_{t}(x, y)\right\}>0,
\end{aligned}
$$

where if $\left\{\boldsymbol{G}_{t}\right\}$ is uniformly lazy, as in (1.12), then in particular $(x, x) \in E$ for all $x \in V$. For uniformly elliptic and lazy $\left\{\boldsymbol{G}_{t}\right\}$, set $\bar{\alpha}:=\alpha_{l} \wedge \alpha_{e}$ (with $\bar{\alpha}=\alpha_{e}$ when CSRW concerned).

Theorem 1.2. [two-sided Gaussian estimates for CSRW] Consider CSRW associated with (1.1) such that $t \mapsto \pi_{t}(x) \in \mathcal{M}_{+}(V)$ is non-decreasing. Assume, in the sense of Definition 1.1, that $\left\{\boldsymbol{G}_{t}\right\}$ is uniformly elliptic with constant $\bar{\alpha}$, of uniform volume 
growth $v(r)$ with doubling constant $\mathrm{C}_{\mathrm{v}}$, satisfying the uniform Poincaré inequality with constant $\mathrm{C}_{\mathrm{P}}$. Then there exists $\mathrm{C}_{\star}=\mathrm{C}_{\star}\left(\mathrm{C}_{\mathrm{v}}, \bar{\alpha}, \mathrm{C}_{\mathrm{P}}\right)$ finite, such that for $\mu_{s, t}$ of (1.8), and all $t-s \geq d(x, y)$,

$$
\frac{\mathrm{C}_{\star}^{-1} \mu_{s, t}(y)}{v(\sqrt{t-s})} \exp \left(-\frac{\mathrm{C}_{\star} d(x, y)^{2}}{t-s}\right) \leq K_{s, t}(x, y) \leq \frac{\mathrm{C}_{\star} \mu_{s, t}(y)}{v(\sqrt{t-s})} \exp \left(-\frac{d(x, y)^{2}}{\mathrm{C}_{\star}(t-s)}\right) .
$$

Theorem 1.2 is a direct consequence of Theorem [1.6 and the detailed Theorem 1.8, with the latter also providing our results in the more technically involved discrete time setting. Note that if $\pi_{t}(\cdot)=\pi_{0}(\cdot)$ is independent of $t$, then also $\mu_{s, t}(y)=\pi_{0}(y)$ for all $t \geq s$ (see Remark [1.9), and our Gaussian transition density estimates take the usual form of the time-homogeneous setting. More generally, the same applies whenever $\left\{\mu_{s, t}\right\}$ of (1.8) are $c$-stable with respect to the strictly positive $\sigma$-finite measures $\pi_{s}$ on $V$, as in [SZ2, Defn 1.10]. That is, whenever for some $c$ finite

$$
c^{-1} \leq \frac{\mu_{s, t}(y)}{\pi_{s}(y)} \leq c, \quad \forall t \geq s \geq 0, \quad y \in V .
$$

Subject to such $c$-stability, considering (1.6)-(1.7) for $y=x$ yields that

$$
\int_{s}^{\infty} K_{s, t}(x, x) d t<\infty \quad \Longleftrightarrow \quad \int_{0}^{\infty} \frac{d t}{\pi_{s}(\mathbb{B}(x, \sqrt{t}))}<\infty .
$$

Starting at $X_{s}=x$, the LHS of (1.16) amounts to a finite expected total occupation time of state $x$, hence its transience for either CSRW or DTRW $\left\{t \mapsto \boldsymbol{G}_{t}\right\}$. If in addition $\sup _{s}\left\{h_{s}\right\}>0$ implies $\inf _{s}\left\{h_{s}\right\}>0$ for $h_{s}:=\mathbb{P}\left(X_{t} \neq x, \forall t>s \mid X_{s}=x\right)$, it thereby answers the transience versus recurrence question raised in ABGK. We believe that $\left\{\mu_{s, t}\right\}$ of Theorem 1.2 (and for DTRW as in Theorem (1.8) are all within a uniform constant of $\pi_{0}$. That is,

Conjecture 1.3. If $\left\{\boldsymbol{G}_{t}\right\}$, of non-decreasing $t \mapsto \pi_{t}(x)$, is uniformly elliptic, of uniform volume growth $v(r)$ with $v(r)$ doubling, and satisfies the uniform Poincaré inequality, then for the corresponding CSRW or uniformly lazy DTRW, $\inf _{t, x}\left\{\mu_{0, t}(x)\right\} \geq 1 / c\left(\mathrm{C}_{\mathrm{P}}, \mathrm{C}_{\mathrm{v}}, \bar{\alpha}\right)>0$.

In proving Theorem 1.2, our steps are (I) first establishing an on-diagonal upper bound (i.e. GHKU for $x=y$ ), (II) proving the full GHKU, but without the term $\mu_{s, t}$, (III) establishing a parabolic Harnack inequality (see Theorem 1.6), and (IV) obtaining the two sided estimates stated from the Harnack inequality and a-priori weaker Gaussian upper bound. This road-map is wellestablished in the literature of heat kernel estimates. However, in the time-varying setting each step requires overcoming difficulties brought by the changing conductances, in particular, by the lack of reversibility. We discuss below in more details our methods for each step.

Our approach to on-diagonal upper bound is through the so called the Nash profile (for its definition see (1.18)). In the time homogeneous setting, Coulhon [C1, C2, C3] systematically derives sharp upper bound on $\left\|K_{0, n}\right\|_{1 \rightarrow \infty}$ out of Nash type inequalities. Our on-diagonal upperbound, namely the RHS of (1.6) for $x=y$, is a special case of the general framework of Section 2. where time dependent reference measures such as $\pi_{n}$ or $\mu_{n}:=\mu_{0, n}$ of (1.8), are used to obtain these upper bounds from the corresponding Nash profiles. We present this method, which is of independent interest, for the case of DTRW. It can be worked out directly for CSRW. Alternatively, the CSRW results can be deduced from the corresponding ones for DTRW, as we do here.

Recall that for Markov operator $K$ on $V$ and its invariant $\sigma$-finite measure $\pi$, the map

$$
f \mapsto(K f)(x):=\sum_{y \in V} K(x, y) f(y),
$$


satisfies $(K f)^{2} \leq K f^{2}$ for bounded $f$, thereby extending to non-expanding map on $L^{2}(\pi)$ having the non-negative definite Dirichlet form

$$
\mathcal{E}_{K, \pi}(f, g):=\langle f-K f, g\rangle_{\pi}
$$

with $L^{2}(\pi) \subseteq \operatorname{Dom}\left(\mathcal{E}_{K, \pi}\right)$. The Nash profile of such $(K, \pi)$ is

$$
\mathcal{N}_{K, \pi}(\mathrm{t}):=\sup \left\{\frac{\|f\|_{L^{2}(\pi)}^{2}}{\mathcal{E}_{K, \pi}(f, f)}: 0<\|f\|_{L^{1}(\pi)}^{2} \leq \mathrm{t}\|f\|_{L^{2}(\pi)}^{2}<\infty\right\} .
$$

Since $\mathrm{t} \mapsto \mathcal{N}_{K, \pi}(\mathrm{t})$ is non-decreasing, setting $\mathcal{N}_{K, \pi}(\infty)=\infty$ yields Nash inequality

$$
\|f\|_{L^{2}(\pi)}^{2} \leq \mathcal{N}_{K, \pi}\left(\|f\|_{L^{1}(\pi)}^{2} /\|f\|_{L^{2}(\pi)}^{2}\right) \mathcal{E}_{K, \pi}(f, f),
$$

for any non-zero $f \in L^{2}(\pi)$. Further, by Cauchy-Schwarz inequality,

$$
\|f\|_{L^{1}(\pi)}^{2} \leq \pi(\operatorname{supp} f)\|f\|_{L^{2}(\pi)}^{2}
$$

so $\mathcal{N}_{K, \pi}(\mathrm{t})$ plays the role of $L^{2}$-isoperimetric profile, where $\mathrm{t}$ acts as the volume.

With the uniform Poincaré inequality providing an explicit upper bound on the Nash profile of weighted graph $\boldsymbol{G}_{t}$ in terms of the doubling function $v(r)$ of (1.11) (see our derivation of (2.21)), the application of Section 2 most relevant here is as follows (c.f. Lemma 2.8).

Theorem 1.4. Suppose $K_{t}$ have reversible measures $\pi_{t} \in \mathcal{M}_{+}(V)$ with $t \mapsto \pi_{t}(x)$ non-decreasing for each $x \in V$ and the non-decreasing $\mathrm{s} \mapsto N(\mathrm{~s})$ is such that for some finite $\mathrm{C}_{\mathrm{n}}, \mathrm{s}_{0}$,

$$
N(\mathrm{~s}) \geq \sup _{t}\left\{\mathcal{N}_{K_{t}^{2}, \pi_{t}}(\mathrm{~s})\right\}, \quad \inf _{\mathrm{s} \geq \mathrm{s}_{0}}\left\{\frac{N\left(\mathrm{C}_{\mathrm{n}} \mathrm{s}\right)}{N(\mathrm{~s})}\right\} \geq 2 .
$$

(a). For the dynamics (1.3) and any $s \leq t$ one has the on-diagonal upper bound

$$
\sup _{x, y \in V}\left\{\frac{K_{s, t}(x, y)}{\pi_{t}(y)}\right\} \leq \mathrm{C}_{\mathrm{n}}^{\prime} \psi\left(\frac{t-s}{3}\right),
$$

for $\psi(\mathrm{t}):=1 / F^{-1}\left(\mathrm{t} ; c_{\star}, N(\cdot)\right)$, and some $\mathrm{C}_{\mathrm{n}}^{\prime}=\mathrm{C}_{\mathrm{n}}^{\prime}\left(\mathrm{C}_{\mathrm{n}}, \mathrm{s}_{0} / c_{\star}\right)<\infty$, where $c_{\star}=\underline{\pi_{0}}$ and

$$
F(u ; a, N(\cdot)):=\int_{a}^{u} \frac{N(\mathrm{~s})}{\mathrm{s}} d \mathrm{~s} .
$$

(b). For the dynamics (1.5) replace $\mathcal{N}_{K_{t}^{2}, \pi_{t}}(\mathrm{~s})$ by $2 \mathcal{N}_{K_{t}, \pi_{t}}(\mathrm{~s})$ in (1.19), with the RHS of (1.20) having the expectation over $\frac{1}{3}$ Poisson $(2(t-s))$ law of the corresponding $\psi(\cdot)$.

We next remark on related works [SZ1, SZ2] and [DHMP]. General time-inhomogeneous transitions $\left\{K_{k, n}(x, y)\right\}$ that satisfy (1.3) for some finite state space $V$, are considered in [SZ1, SZ2]. Aiming at merging for such transitions, namely the suitable convergence to zero of $\mid K_{0, n}(x, y)-$ $K_{0, n}\left(x^{\prime}, y\right) \mid$ as $n \rightarrow \infty$, [SZ1, SZ2] develop in this context analytic tools such as the Nash and logSobolev inequalities, where a key assumption of [SZ2] is that the Markov transitions $\left\{K_{0, n}\right\}$ yield $\mu_{0, n}$ as in (1.8) which are $c$-stable with respect to some $\pi_{0} \in \mathcal{M}_{+}(V)$. The $c$-stability condition (1.15) is in general difficult to verify, as it requires fine understanding of the transition probabilities $K_{s, t}$. Theorem 1.4, and more generally Proposition 2.4, are phrased in a general framework that allows more flexible choices of reference measures, see Example 2.2 and 2.3. In particular, under the non-decreasing assumption on $t \mapsto \pi_{t}(x)$, we do not require $c$-stability. The method of Nash profiles applies also when $N(\cdot)$ grows too slowly for the RHS of (1.19), for example when $N\left(e^{\mathrm{s}}\right)$ is a doubling function (see Remark 2.6). 
Under the same assumption that $n \mapsto \pi_{n}(x)$ is non-decreasing, evolving sets are used in DHMP] for deriving the heat kernel on-diagonal upper bound (ie. GHKU for $x=y$ ) for uniformly lazy DTRW from $L^{1}$-isoperimetry property of $\left\{\boldsymbol{G}_{n}\right\}$. Via a different approach, Theorem 1.4 strengthens the main result of [DHMP], see Example 2.10, More precisely, we recover the same on-diagonal upper bound in the more general setting of (1.3), while replacing the assumed $\kappa_{m} \mathrm{~s}^{-1 / d}$ lower bound on the $L^{1}$-isoperimetric profile for the weighted graph $\boldsymbol{G}_{m}$, with having only $\mathcal{N}_{K_{m}^{2}, \pi_{m}}(\mathrm{~s}) \leq \kappa_{m}^{-2} \mathrm{~s}^{2 / d}$ (c.f. Lemma 2.9 for a comparison between the Nash and $L^{1}$-isoperimetric profiles which follows from the Cheeger inequality). We note in passing that Theorem 1.4 can even be applied to certain non-local Markov transition kernels, as demonstrated by Example 2.11.

Due to lack of reversibility for $K_{s, t}$ when $t>s$, the off-diagonal upper bound is technically more involved. Nevertheless, in Section 3 we adapt the technique of [HS, Section 2] for deriving off-diagonal Gaussian upper bounds via complex interpolation. The complex interpolation method requires two input bounds: a bound on the $2 \rightarrow \infty$ norm of $K_{s, t}$ and a bound on the $2 \rightarrow 2$ norm of the perturbed kernel $K_{s, t}^{\theta}$ (see (3.1)) with respect to appropriate reference measures, see Proposition 3.1. The $2 \rightarrow \infty$ norm bound is provided by the Nash profile method in Section 2. The bound on $2 \rightarrow 2$ norm is often referred to as the Gaffney lemma, which we prove for time non-decreasing $\pi_{t}$ in Lemma 3.5 (based on Lemma 3.4). The complex interpolation method is especially suited for our purpose, since it never invokes reversibility and incorporates well having time dependent reference measures $\pi_{s}$ (for each term $K_{s}$ ).

So far what we have discussed applies equally well to CSRW and DTRW. The GHKL turns out to be more difficult without reversibility, even if just to obtain an on-diagonal lower estimate. Specializing the setting of Theorem 1.4. back to that of weighted graphs $\left\{\boldsymbol{G}_{t}\right\}$ that satisfy the uniform Poincaré inequality and uniform volume doubling, our approach is thus to first establish a Parabolic Harnack Inequality (PHI), then derive the full two-sided GHKE from it. To this end, we first introduce the notations needed for stating such parabolic Harnack inequality. We call $u(\cdot, \cdot) \geq 0$ on a time-space cylinder

$$
Q:=Q\left(t_{1}, t_{2} ; z, R\right)=\left[t_{1}, t_{2}\right] \times \mathbb{B}(z, R)
$$

a (non-negative) solution to the (backward) heat equation, if

$$
\partial_{-s} u(s, x)=\sum_{y} K_{s}(x, y) u(s, y)-u(s, x), \quad \forall(s, x) \in Q,
$$

for some non-negative boundary values (for $u$ ) outside $Q$. For bounded range $K_{s}$ we have that (1.23) holds on $\mathbb{B}\left(z, R-r_{0}\right)$ even when restricting the sum to $\mathbb{B}(z, R)$. Here $r_{0}=1$, so such solution is uniquely specified by $\left\{u(s, x): d(z, x)=R\right.$ or $\left.s=t_{2}\right\}$. For discrete time we take $s \in \mathbb{N}$ and $\partial_{-s} u(s, \cdot):=u(s-1, \cdot)-u(s, \cdot)$, whereas for CSRW we assume WLOG that $s \mapsto u(s, x)$ is absolutely continuous, so $\partial_{-s} u$ exists a.e. and (1.23) interpreted as a distributional identity via integration by parts.

We say that the PHI holds for (1.23), if for any $0<\theta_{1} \leq \theta_{2}<\theta_{3} \leq \theta_{4}$ some $\gamma=\gamma\left(\theta_{i}\right) \in(0,1)$, any $T \geq\left(\theta_{4} R\right)^{2}$ and solution $u$ of (1.23) on time-space cylinder $Q\left(T-\left(\theta_{4} R\right)^{2}, T ; z, 8 R\right)$, we have

$$
\left(\theta_{2 i-1} R\right)^{2}<\tau_{i} \leq\left(\theta_{2 i} R\right)^{2}, x_{1}, x_{2} \in \mathbb{B}(z, R) \quad \Longrightarrow \quad u\left(T-\tau_{2}, x_{2}\right) \geq \gamma u\left(T-\tau_{1}, x_{1}\right),
$$

further restricting (1.24) in the discrete case to $\tau_{2} \geq \tau_{1}+d\left(x_{1}, x_{2}\right)$.

Remark 1.5. If $u(\cdot, \cdot)$ satisfies (1.23) then so does $a u(\cdot, \cdot)+b$. Considering $b \downarrow 0$ we deduce that it suffices to prove the PHI only for strictly positive solutions. 
Recall [Del] that for CSRW on time-invariant conductances, the PHI is equivalent to uniformly elliptic conductances satisfying both the PI and VD. Our next result extends this to time-varying, non-decreasing vertex conductances $t \mapsto \pi_{t}(\cdot)$.

Theorem 1.6. [parabolic Harnack inequality]

Suppose $\left\{\boldsymbol{G}_{t}\right\}$ of non-decreasing $t \mapsto \pi_{t}(x) \in \mathcal{M}_{+}(V)$ and $\mathrm{C}_{0}:=\sup _{t, x}\left\{\frac{\pi_{t}(x)}{\pi_{0}(x)}\right\}$ finite, is uniformly elliptic, satisfying the uniform volume doubling condition and the uniform Poincaré inequality. Then, the PHI holds for the continuous time heat equation (1.23) and some $\gamma=\gamma\left(\mathrm{C}_{\mathrm{P}}, \mathrm{C}_{\mathrm{D}}, \alpha_{e}, \mathrm{C}_{0}\right)$ positive.

For time-invariant conductances the PHI implies Hölder regularity of (non-negative) solutions of the heat equation (see [Del, Pages 227-228]). This extends to our setting, yielding the Hölder regularity of $(s, x) \mapsto K_{s, t}(x, z)$ under the conditions of Theorem 1.6.

Proposition 1.7. The PHI implies existence of $h(\gamma)>0$ such that for any $z \in V, R \geq 1, T \geq 4 R^{2}$ and solution $u \geq 0$ of (1.23) on $Q=Q\left(T-4 R^{2}, T ; z, 8 R\right)$, if $y_{j} \in \mathbb{B}(z, R)$ and $\left(T-s_{j}\right) \in\left[R^{2}, 4 R^{2}\right]$, $j=1,2$, then

$$
\left|u\left(s_{2}, y_{2}\right)-u\left(s_{1}, y_{1}\right)\right| \leq(4 / R)^{h}\left(\left|s_{2}-s_{1}\right|^{1 / 2} \vee d\left(y_{1}, y_{2}\right)\right)^{h} \sup _{Q}\{u\}
$$

Parabolic Harnack inequalities, Gaussian estimates and Hölder regularity of solutions of the heat equation have a long history. Aiming at a-priori Hölder continuity for solutions of the heat equation

$$
\partial_{t} u(t, x)=\mathcal{L} u(t, x), \quad t \geq 0, x \in \mathbb{M} \subseteq \mathbb{R}^{d},
$$

on a Riemannian manifold $\mathbb{M}$, with a divergence form operator

$$
\mathcal{L} u:=\sum_{i, j=1}^{d} \partial_{x_{i}}\left(a_{i j}(t, x) \partial_{x_{j}} u\right)
$$

having symmetric, measurable, uniformly elliptic matrix of coefficients $\left\{a_{i j}(\cdot)\right\}$, the study of heat kernel asymptotics for the corresponding diffusion on $\mathbb{M}$, goes back at least to works of De Giorgi, Nash, Moser in mid-century. The characterization of two-sided Gaussian Heat Kernel Estimates (GHKE), for the solutions of (1.26) (namely, the diffusion analog of VSRW), in terms of Poincaré Inequality (PI), plus the volume doubling (VD) property, and their equivalence to the Parabolic Harnack Inequality (PHI), are established independently by [Gr, $\mathrm{SC}$. Such results have later been derived in [St1, St2] for time-dependent, strongly local Dirichlet forms on metric measure spaces (subject to the existence of a time-invariant Radon measure in the underlying topological space). However, strongly local Dirichlet forms as in [St1, St2] can have no jumps (nor killing). In particular, this assumption excludes the uniformly elliptic (and lazy) random walks on a (static) graph $\boldsymbol{G}_{0}$, for which such equivalence between GHKE, PHI and PI+VD is proved in [Del]. See also [BC], which proves a similar equivalence for CSRW on non-elliptic (static) graph $\boldsymbol{G}_{0}$, when the GHKE, PHI and $\mathrm{PD}+\mathrm{VI}$ are suitably restricted (to large balls). One direction we pursue here, is to extend this graph part of the theory, by obtaining the GHKU (with $\mu_{s, t}$ replaced by $\pi_{t}$ ), for both dynamics of (1.3) and (1.5), allowing for genuinely time-varying, non-decreasing $\left\{\pi_{t}(x): x \in V\right\}$. In a related context, the two-sided Gaussian heat kernel estimates are already provided in [DD, GOS] for continuous-time symmetric rate random walks on $\mathbb{Z}^{d}$ having time-dependent, uniformly elliptic jump rates $c_{t}(x, y)$ (i.e. the so-called variable speed random walk vsRw; c.f. [MO] for the same in certain degenerate cases lacking uniform ellipticity). Indeed, the treatment of time-varying VSRW is much simpler than both DTRW and CSRW since any VSRW has the time-invariant reversing measure $\left\{\pi_{t}(x)=1: x \in V, t \geq 0\right\}$. Similar reversible situation applies in [CGZ] where two-sided 
Gaussian heat kernel bounds are stated (without a detailed proof), for the DTRW of (1.3), provided $\left\{\pi_{n}(x)=\pi_{0}(x)\right\}$ is constant in time and a uniform Sobolev inequality holds.

The approach to establish PHI from volume doubling and Poincaré inequalities, as in $[\mathrm{Gr}]$ and [SC] (that we adapt for proving Theorem [1.6 in Section 44), relies on taking the time derivative of the logarithm of the heat kernel. Having a discrete-time version of such a step, is a well known open challenge. This difficulty can be circumvented by first deriving the HKE-s and then deducing the PHI from them, see [FS]. Indeed, for time-invariant conductances, as in [Del], one compares the transition probabilities of DTRW to those of the CSRW, thereby obtaining the Gaussian estimates for the DTRW, which in turn yield the PHI. However, such a comparison with the CSRW is not available in our time-varying setting. Alternatively, in [HS2, Hebisch and Saloff-Coste prove the PHI for discrete-time dynamic, directly, from a scale invariant elliptic Harnack inequality and local Sobolev inequalities. Unfortunately, it is unclear what should be the analogous elliptic objects to study in the time-varying setting. For these reasons, we are only able to establish parabolic Harnack inequalities in the case of CSRW.

We summarize our main results for both CSRW and DTRW as follows. As mentioned before, under the assumption of uniform Poincaré inequalities, uniform ellipticity, and uniform volume growth doubling conditions, we derive a GHKU without the term $\mu_{s, t}$ for both CSRW and uniformly lazy DTRW; then the improved GHKU with respect to $\mu_{s, t}$ and the matching GHKL are both obtained as a consequence of the PHI.

Theorem 1.8. [two-sided Gaussian HKE]

Consider either CSRW or a uniformly lazy DTRW associated with (1.1), for non-decreasing $t \mapsto \pi_{t}(x)$ and $\left\{\boldsymbol{G}_{t}\right\}$ of uniform volume growth $v(r)$ with $v(r)$ doubling.

(a). Let $I(r)=r^{2}$ on $[0,1]$, and for $r>1$ set $I(r)=r(\log r+1)$ for the CsRW, while $I(r)=\infty$ for the DTRW. Then, the uniform Poincaré inequality yields that for some finite $C=C\left(\mathrm{C}_{\mathrm{P}}, \mathrm{C}_{\mathrm{v}}, \alpha_{l}\right)$,

$$
K_{s, t}(x, y) \leq \frac{C}{v(\sqrt{t-s})} \exp \left\{-\frac{(t-s)}{C} I\left(\frac{d(x, y)}{t-s}\right)\right\}, \quad \forall x, y \in V, t \geq s \geq 0 .
$$

(b) Suppose (1.27) and the PHI hold (so for DTRW, the graphs $\left\{\boldsymbol{G}_{t}\right\}$ are uniformly elliptic and lazy). Then, for $\mu_{s, t}$ of (1.8) , some $\mathrm{C}_{\star}=\mathrm{C}_{\star}\left(C, \gamma, \mathrm{C}_{\mathrm{v}}, \bar{\alpha}\right)$ finite and all $t-s \geq d(x, y)$,

$$
\frac{\mathrm{C}_{\star}^{-1} \mu_{s, t}(y)}{v(\sqrt{t-s})} \exp \left(-\frac{\mathrm{C}_{\star} d(x, y)^{2}}{t-s}\right) \leq K_{s, t}(x, y) \leq \frac{\mathrm{C}_{\star} \mu_{s, t}(y)}{v(\sqrt{t-s})} \exp \left(-\frac{d(x, y)^{2}}{\mathrm{C}_{\star}(t-s)}\right) .
$$

Remark 1.9. In the discrete time setting of (1.3), if $t \mapsto \pi_{t}(x)$ is non-decreasing at each $x \in V$, then pointwise $\pi_{s} K_{s}=\pi_{s} \leq \pi_{s+1}$, hence $\mu_{s, t}=\left(\pi_{s} K_{s}\right) K_{s+1, t} \leq \mu_{s+1, t}$ for $\mu_{s, t}$ of (1.8). That is,

$$
\mu_{t}(x):=\mu_{0, t}(x) \leq \mu_{s^{\prime}, t}(x) \leq \mu_{s, t}(x) \leq \mu_{t, t}(x)=\pi_{t}(x), \quad \forall t \geq s \geq s^{\prime} \geq 0, \quad x \in V
$$

To verify that the same applies in the setting of the continuous time evolution (1.4), recall that $\mu_{s, t}$ is then the expected value over $N \sim \operatorname{Poisson}(t-s)$ and jump times $s<T_{1}^{\prime}<\cdots<T_{N}^{\prime} \leq t$ of the value $\mu_{0, N}^{(\omega)}$ for a discrete time dynamic starting at $\pi_{s}$ and using the random $\left\{K_{T_{m}^{\prime}}\right\}$ in (1.3). With $s^{\prime} \in\left(T_{L}^{\prime}, T_{L+1}^{\prime}\right) \cap(s, t)$ for some $0 \leq L \leq N$, clearly $\mu_{s^{\prime}, t}$ exceeds the expected value of $\mu_{L, N}^{(\omega)}$ for the corresponding discrete time dynamic, so by the monotonicity of the expectation, (1.29) applies also for any continuous time evolution (1.4) with non-decreasing $t \mapsto \pi_{t}(\cdot)$. By the same reasoning, for both DTRW and CSRW, if $t \mapsto \pi_{t}(x)$ is non-increasing, then so is $s \mapsto \mu_{s, t}(x)$. In particular, for the special case of $\pi_{t}(x)=\pi(x)$ independent of $t$ we have that $\mu_{s, t}=\pi$ and Theorem 1.8 recovers (under uniform Poincaré inequality and uniform volume growth $v(r)$ with $v(r)$ doubling), the Gaussian upper bound for DTRW stated in [CGZ, Sec. 7]. 
In view of (1.29), upon verifying Conjecture 1.3 the RHS of (1.16) should provide a criterion for transience/recurrence of CSRW in terms of the volume growth of $\boldsymbol{G}_{0}$ (and upon proving the discrete time PHI, the same would apply for uniformly elliptic and lazy DTRW).

Remark 1.10. Without monotonicity of $n \mapsto \pi_{n}$, even for $\left\{\pi_{n}\right\}$ that are $c$-stable WRT the function $\nu_{0}(x) \equiv 1$ on $\mathbb{G}=\mathbb{Z}_{\geq 0}$, the reference $\mu_{n}=\mu_{0, n}$ may be non-comparable with $\pi_{n}$. For example, fixing $\eta, \epsilon>0$ let $\pi_{n}(x, x+1)=1+(-1)^{n+x} \eta$ with $\pi_{n}(x, x)=1+\epsilon \mathbf{1}_{\{n+x \text { odd }\}}$ when $x>0$ and $\pi_{n}(0,0)=\pi_{n}(2,2) \pi_{n}(0,1) / 2$ (to assure that $K_{n}(0,0)=K_{n}(2 x, 2 x)$ for any $\left.n, x\right)$. Classifying states into types $A$ or $B$ according to $n+X_{n}$ being even or odd, respectively, yields an $\{A, B\}$-valued homogeneous Markov chain of invariant measure $\left[m_{A}, m_{B}\right]=[3(1+\epsilon), 3+\epsilon]$. The process $\left\{X_{n}\right\}$ has drift $\eta$ at the $A \mapsto A$ moves with opposite drift at $B \mapsto B$ moves. Consequently, $\left\{X_{n}\right\}$ has asymptotic speed $v=\eta \epsilon /(3+2 \epsilon)$ to the right. In particular, for some $C$ finite and any $y \in \mathbb{Z}_{\geq 0}$ we have for all $n \geq C y / v$, the fast decay

$$
\mu_{n}(y)=\sum_{x \geq 0} K_{0, n}(x, y) \leq C \sqrt{n} e^{-(n v-y)^{2} /(C n)} .
$$

In Section 6 we treat a perturbative regime. Specifically, we show that the GHKU of Theorem 1.4 and Theorem 1.8(a) apply as soon as $t \mapsto e^{a_{t}} \pi_{t}(\cdot)$ is non-decreasing for some non-decreasing $t \mapsto a_{t}$ such that

$$
A:=\sup _{t \geq 0}\left\{a_{2 t+1}-a_{t}\right\}<\infty .
$$

Further, we get the matching GHKL if (1.30) applies for

$$
a_{t}=\sup _{\ell} \sup _{0=s_{0}<\cdots<s_{\ell}=t}\left\{\sum_{i=0}^{\ell-1} \rho_{\boldsymbol{\pi}}\left(s_{i}, s_{i+1}\right)\right\}, \quad \rho_{\boldsymbol{\pi}}\left(s, s^{\prime}\right):=\sup _{x \in V}\left|\log \frac{\pi_{s^{\prime}}(x)}{\pi_{s}(x)}\right|
$$

(considering for DTRW only $s_{i} \in \mathbb{N}$ ). In particular, for CSRW with absolutely continuous $s \mapsto \pi_{s}(x)$ we have in (1.31) absolutely continuous $a_{t}$ such that a.e. $\partial_{t} a_{t}=\sup _{x \in V}\left|\partial_{t} \log \pi_{t}(x)\right|$.

Proposition 1.11. Suppose $\left\{\boldsymbol{G}_{t}\right\}$ uniformly elliptic of uniform volume growth $v(r)$ with $v(r)$ doubling, has the uniform Poincaré inequality and some $\left\{a_{t}\right\}$ satisfies (1.30)-(1.31).

(a) The GHKU holds for either CSRW or uniformly lazy DTRW, without $\mu_{s, t}(\cdot)$, in (1.6), and with some $C=C\left(A, \mathrm{C}_{\mathrm{P}}, \mathrm{C}_{\mathrm{v}}, \bar{\alpha}\right)$ finite.

(b) The matching GHKL holds for CSRW, and subject to the discrete time PHI, also for DTRW.

Remark 1.12. Starting at a uniformly elliptic $\boldsymbol{G}_{0}$ of volume growth $v(r)$ with $v(r)$ doubling that satisfies the Poincaré inequality, Proposition 1.11 yields the matching GHKE for the CSRW on $\pi_{t}(x, y)=\pi_{0}(x, y) e^{h_{t}(x, y)}$, whenever $\sup _{t}\left\{\left\|h_{t}\right\|_{\infty}\right\}$ and $\sup _{t}\left\{(t+1)\left\|\partial_{t} h_{t}\right\|_{\infty}\right\}$ are finite. In particular, this setting allows us to have forever oscillating $t \mapsto \pi_{t}(x)$.

While in Proposition 1.11 we have $a_{n}=O(\log n)$, we next show that no such Gaussian estimates hold universally when $a_{n}$ grows as $O\left(n^{1 / 2+\iota}\right)$ for some $\iota>0$. It is interesting to find a sharp threshold in the context of Proposition 1.11, and in particular to determine whether $a_{n} \leq O\left(n^{1 / 2}\right)$ suffices for such Gaussian density bounds.

Proposition 1.13. For any $\iota>0$, there exist uniformly bounded, uniformly elliptic, and uniformly lazy, time-varying edge-conductances on $\mathbb{Z}$, with

$$
\limsup _{n \rightarrow \infty}\left\{n^{-(1 / 2+\iota)} a_{n}\right\}<\infty,
$$

such that neither (1.6) nor (1.7) hold for the corresponding DTRW $\left\{X_{n}\right\}$. 
The rest of the paper is organized as follows. Section 2 explores a general framework using evolving reference measures for obtaining on-diagonal transition probability upper bounds from Nash profiles of underlying graphs and can be read independently of the rest of this paper. Section 3 adapts to our time-inhomogeneous setting the perturbation-interpolation technique of [HS, Section 2] for deriving off-diagonal upper bounds (from a given on-diagonal upper bound), concluding with the GHKU of Theorem 1.8(a). We establish in Section 4 the PHI of Theorem 1.6] and the regularity estimate of Proposition 1.7. Section 5 then complete the derivation of Theorem 1.8, whereas Section 6] deals with the perturbative regime of Propositions 1.11 and 1.13.

\section{NASh Inequalities}

Recall the definition of the Nash profile $\mathcal{N}_{K, \pi}$ as in (1.18). Given a dynamic (1.3) for Markov kernels $\left\{K_{n}\right\}$ the Nash method relies on finding auxiliary Markov kernels $Q_{n}$ reversible for some $\nu_{n} \in \mathcal{M}_{+}(V)$, having useful Nash profiles $\mathcal{N}_{Q_{n}, \nu_{n}}(\mathrm{t})$ as well as the following contraction properties.

Assumption 2.1. Markov kernels $Q_{n}$ are reversible for $\nu_{n} \in \mathcal{M}_{+}(V)$ and for any $f \in L^{2}\left(\nu_{n}\right)$

$$
\left\|K_{n} f\right\|_{L^{2}\left(\nu_{n-1}\right)}^{2}+\mathcal{E}_{Q_{n}, \nu_{n}}(f, f) \leq\|f\|_{L^{2}\left(\nu_{n}\right)}^{2} .
$$

Further, for any $f \in L^{1}\left(\nu_{n}\right)$

$$
\left\|K_{n} f\right\|_{L^{1}\left(\nu_{n-1}\right)} \leq\|f\|_{L^{1}\left(\nu_{n}\right)} .
$$

In particular $K_{n}$ must be a bounded operator from $L^{p}\left(\nu_{n}\right)$ to $L^{p}\left(\nu_{n-1}\right)$ for $p=1,2$.

We proceed to provide two canonical examples (of pairs $Q_{n}, \nu_{n}$ ), for which Assumption 2.1 holds.

Example 2.2. If $\nu \in \mathcal{M}_{+}(V)$ and Markov kernel $K$ are such that $\mu=(\nu K) \in \mathcal{M}_{+}(V)$, then $f \mapsto$ $K f$ of (1.17) extends uniquely to the non-negative, bounded linear map $K_{\mu \rightarrow \nu}: L^{2}(\mu) \rightarrow L^{2}(\nu)$. Its dual $K_{\nu \rightarrow \mu}^{\star}: L^{2}(\nu) \rightarrow L^{2}(\mu)$ then satisfies

$$
\left\langle h, K_{\nu \rightarrow \mu}^{\star} g\right\rangle_{\mu}=\langle K h, g\rangle_{\nu}, \quad \forall g \in L^{2}(\nu), h \in L^{2}(\mu),
$$

with the self-adjoined non-negative operator $Q=K^{\star} K$ such that

$$
\langle h, Q f\rangle_{\mu}=\langle K h, K f\rangle_{\nu}, \quad \forall f, h \in L^{2}(\mu) .
$$

Taking $h=\delta_{x}$ and $f=\delta_{y}$ in (2.4), we further see that

$$
Q(x, y):=\frac{1}{\mu(x)} \sum_{z} \nu(z) K(z, x) K(z, y),
$$

is a $\mu$-reversible, Markov transition kernel. Further, for $f \in L^{2}(\mu)$ we have from (2.4) that

$$
\mathcal{E}_{Q, \mu}(f, f)=\|f\|_{L^{2}(\mu)}^{2}-\|K f\|_{L^{2}(\nu)}^{2} .
$$

Since $\mu=(\nu K)$ we also have that

$$
\begin{aligned}
\|K f\|_{L^{1}(\nu)}=\sum_{x} \nu(x)\left|\sum_{y} K(x, y) f(y)\right| & \leq \sum_{x, y} \nu(x) K(x, y)|f(y)| \\
& =\sum_{y}(\nu K)(y)|f(y)|=\|f\|_{L^{1}(\mu)} .
\end{aligned}
$$

Thus, Assumption 2.1 holds for $\mu_{n}:=\mu_{0, n}$ of (1.8) and the corresponding $\mu_{n}$-reversible Markov kernels $Q_{n}:=K_{\mu_{n-1} \rightarrow \mu_{n}}^{\star} K_{n}$, provided

$$
\mu_{n} \in \mathcal{M}_{+}(V), \quad n=0,1,2, \ldots
$$


Starting at any $\mu_{0} \in \mathcal{M}_{+}(V)$, one has (2.8) for uniformly lazy walks, where $\underline{\mu_{n}} \geq \widehat{\alpha} \underline{\mu_{n-1}}$ are strictly positive, since

$$
\widehat{\alpha}:=\inf _{n, y} \sum_{x} K_{n}(x, y) \geq \alpha_{l}
$$

of (1.12), and by induction having per $n, y$ only finitely many $x \in V$ for which $K_{n}(x, y)>0$ guarantees the finiteness of $\mu_{n}(y)$.

If $\left\{\mu_{0, n}\right\}$ are $c$-stable (see (1.15)), then similarly to the considerations of [SZ2], one may estimate the Nash profile $\mathcal{N}_{Q_{n}, \mu_{n}}(\mathrm{t})$ in terms of say $\mathcal{N}_{Q_{1}, \mu_{1}}(\cdot)$. However, not withstanding Conjecture 1.3 we have no systematic way towards such $c$-stability, without which we have little control on $\mathcal{N}_{Q_{n}, \mu_{n}}(\mathrm{t})$.

Example 2.3. If the Markov kernel $K$ has an invariant measure $\pi \in \mathcal{M}_{+}(V)$ then considering Example 2.2 for $\nu=\pi$ results with $\mu=(\nu K)=\pi$. Suppose now that $K_{n}$ have invariant measures $\pi_{n} \in \mathcal{M}_{+}(V)$ such that $n \mapsto \pi_{n}(x)$ are non-decreasing. Then $n \mapsto\|K f\|_{L^{p}\left(\pi_{n}\right)}$ are non-decreasing for $p=1,2$, hence from (2.6)-(2.7) we deduce that Assumption 2.1 holds for $\nu_{n}=\pi_{n}$. If further $K_{n}$ is $\pi_{n}$-reversible, as in Theorem 1.4, then from (2.5) we see that $Q_{n}=K_{n}^{2}$.

In view of Example 2.3, part (a) of Theorem 1.4 is a special case of our next proposition dealing with the more general setting of Assumption 2.1.

Proposition 2.4. Suppose in addition to Assumption 2.1, that for non-decreasing $\mathrm{s} \mapsto N(\mathrm{~s})$

$$
\begin{gathered}
N(\mathrm{~s}) \geq \sup _{k}\left\{\mathcal{N}_{Q_{k}, \nu_{k}}(\mathrm{~s})\right\}, \quad \inf _{k}\left\{\underline{\nu_{k}}\right\} \geq c_{\star}>0, \\
\inf _{\mathrm{s} \geq \mathrm{s}_{0}}\left\{\frac{N\left(\mathrm{C}_{\mathrm{n}} \mathrm{s}\right)}{N(\mathrm{~s})}\right\} \geq 2 .
\end{gathered}
$$

Then the bound (1.20) holds with $\nu_{n}$ instead of $\pi_{n}$.

Turning to the proof of Proposition 2.4 note that fixing hereafter $\left\{\nu_{n}\right\} \subseteq \mathcal{M}_{+}(V)$, part of Assumption 2.1 is having bounded operators $K_{n}: L^{2}\left(\nu_{n}\right) \rightarrow L^{2}\left(\nu_{n-1}\right)$, and hence the dual (adjoint) non-negative operators

$$
\left(K_{n}\right)_{\nu_{n-1} \rightarrow \nu_{n}}^{\star}: L^{2}\left(\nu_{n-1}\right) \rightarrow L^{2}\left(\nu_{n}\right)
$$

as in (2.3), using $K_{n}^{\star}$ for $\left(K_{n}\right)_{\nu_{n-1} \rightarrow \nu_{n}}^{\star}$ whenever the choice of $\left\{\nu_{n}\right\}$ is clear. For any $0 \leq m<n$,

$$
K_{m, n}^{\star}:=K_{n}^{\star} K_{n-1}^{\star} \cdots K_{m+1}^{\star},
$$

is the adjoint of $K_{m, n}: L^{2}\left(\nu_{n}\right) \rightarrow L^{2}\left(\nu_{m}\right)$ of (1.3), about which we have the following bound.

Lemma 2.5. Under Assumption 2.1, if $N_{k}(\mathrm{t}) \geq \mathcal{N}_{Q_{k}, \nu_{k}}(\mathrm{t})$ is non-decreasing in $\mathrm{t}$, then for any $0 \leq m \leq n$,

$$
\psi_{n}(n-m)^{\frac{1}{2}} \geq\left\|K_{m, n}\right\|_{L^{1}\left(\nu_{n}\right) \rightarrow L^{2}\left(\nu_{m}\right)}=\left\|K_{m, n}^{\star}\right\|_{L^{2}\left(\nu_{m}\right) \rightarrow L^{\infty}\left(\nu_{n}\right)},
$$

where starting at any $\psi_{n}(0) \geq 1 / \underline{\nu_{n}}$ we inductively set $\psi_{n}(\cdot)$ such that for $F(\cdot)$ of (1.21),

$$
\frac{1}{\psi_{n}(j+1)}:=F^{-1}\left(1 ; \frac{1}{\psi_{n}(j)}, N_{n-j}(\cdot)\right), \quad j=0, \ldots, n-1 .
$$

Proof. Fix $g: V \rightarrow \mathbb{R}$ such that $\|g\|_{L^{1}\left(\nu_{n}\right)}=1$ and let

$$
J_{n}(j):=\left\|K_{m, n} g\right\|_{L^{2}\left(\nu_{m}\right)}^{2} \quad \text { with } \quad j=n-m .
$$

Since $K_{n, n}=I$ we have that

$$
J_{n}(0)=\|g\|_{L^{2}\left(\nu_{n}\right)}^{2} \leq \frac{1}{\underline{\nu_{n}}} \sum_{x}\left[\nu_{n}(x)|g(x)|\right]^{2} \leq \frac{1}{\underline{\nu_{n}}} \leq \psi_{n}(0),
$$


is finite (since $\nu_{n} \in \mathcal{M}_{+}(V)$ ). In particular, here $L^{1}\left(\nu_{n}\right) \subseteq L^{2}\left(\nu_{n}\right) \subseteq L^{\infty}\left(\nu_{n}\right)$ and the identity on the RHS of (2.11) follows by duality between $L^{1}\left(\nu_{n}\right)$ and $L^{\infty}\left(\nu_{n}\right)$. Turning to prove inductively for $j=1,2, \ldots$ the inequality on its LHS, recall that $K_{m-1, n}=K_{m} K_{m, n}$ hence considering (2.1) for $f_{m}:=K_{m, n} g$ in $L^{2}\left(\nu_{m}\right)$ and the definition (1.18) of $\mathcal{N}_{Q_{m}, \nu_{m}} \leq N_{m}$ we find that

$$
J_{n}(j)-J_{n}(j+1)=\left\|f_{m}\right\|_{L^{2}\left(\nu_{m}\right)}^{2}-\left\|K_{m} f_{m}\right\|_{L^{2}\left(\nu_{m-1}\right)}^{2} \geq \mathcal{E}_{Q_{m}, \nu_{m}}\left(f_{m}, f_{m}\right) \geq \frac{\left\|f_{m}\right\|_{L^{2}\left(\nu_{m}\right)}^{2}}{N_{m}(\mathrm{t})}
$$

provided $\mathrm{t}$ is such that

$$
\left\|f_{m}\right\|_{L^{1}\left(\nu_{m}\right)}^{2} \leq \mathrm{t}\left\|f_{m}\right\|_{L^{2}\left(\nu_{m}\right)}^{2} .
$$

Next, iterative consideration of (2.2) down from $n$ to $m$ yields that

$$
\left\|f_{m}\right\|_{L^{1}\left(\nu_{m}\right)} \leq\|g\|_{L^{1}\left(\nu_{n}\right)}=1
$$

hence by the definition of $J_{n}(j)$ we can use $\mathrm{t}=1 / J_{n}(j)$ to deduce that

$$
J_{n}(j)-J_{n}(j+1) \geq H_{n-j}\left(J_{n}(j)\right), \quad j=0, \ldots, n-1,
$$

where $H_{m}(u):=u / N_{m}(1 / u)$. Since $\mathrm{t} \mapsto N_{m}(\mathrm{t})$ is non-decreasing, so is the positive $u \mapsto H_{m}(u)$ and consequently the piece-wise linear interpolation of $J_{n}(\cdot)$ to $[0, n]$ satisfies

$$
-\frac{d}{d u}\left\{J_{n}(u)\right\} \geq H_{n-j}\left(J_{n}(u)\right), \quad \forall u \in(j, j+1) .
$$

It is easy to verify that

$$
-\frac{d}{d u}\left\{\psi_{n}(u)\right\}=H_{n-j}\left(\psi_{n}(u)\right), \quad \forall u \in(j, j+1)
$$

for the continuous interpolation of $\psi_{n}(\cdot)$ of (2.12) according to $\frac{1}{\psi_{n}(u)}:=F^{-1}\left(u-j ; \frac{1}{\psi_{n}(j)}, N_{n-j}(\cdot)\right)$ for $u \in(j, j+1)$, which by (2.13) starts at $\psi_{n}(0) \geq J_{n}(0)$. Thus, the continuous $\psi_{n}(u)-J_{n}(u)$ is non-negative on $[0, n]$ and in particular $J_{n}(j) \leq \psi_{n}(j)$ for all $0 \leq j \leq n$. This holds whenever $\|g\|_{L^{1}\left(\nu_{n}\right)}=1$, so $\psi_{n}(j)$ control the relevant operator norms.

Remark 2.6. With $\underline{\nu_{k}}>0$, Proposition 2.4 is equivalent to the operator norm bound

$$
\left\|K_{m, n}^{\star}\right\|_{L^{1}\left(\nu_{m}\right) \rightarrow L^{\infty}\left(\nu_{n}\right)}=\left\|K_{m, n}\right\|_{L^{1}\left(\nu_{n}\right) \rightarrow L^{\infty}\left(\nu_{m}\right)} \leq \mathrm{C}_{\mathrm{n}}^{\prime} \psi\left(\frac{n-m}{3}\right) .
$$

Even without (2.10), by Lemma 2.5 we get from (2.9) that

$$
\left\|K_{m, n}\right\|_{L^{1}\left(\nu_{n}\right) \rightarrow L^{\infty}\left(\nu_{m}\right)} \leq c_{\star}^{-1 / 2}\left\|K_{m, n}\right\|_{L^{1}\left(\nu_{n}\right) \rightarrow L^{2}\left(\nu_{m}\right)} \leq c_{\star}^{-1 / 2} \psi^{1 / 2}(n-m) .
$$

If $\psi(\mathrm{t}) \leq \psi(\delta \mathrm{t})^{2}$ for some $\delta>0$, this already yields the bound (1.20) of Proposition 2.4 (albeit with $\psi(\delta \cdot)$ instead of $\psi(\cdot / 3))$. For example, replacing the (fast) growth assumption (2.10) by

$$
\sup _{\mathrm{s} \geq c_{\star}}\left\{\frac{N\left(\mathrm{~s}^{2}\right)}{N(\mathrm{~s})}\right\} \leq \mathrm{C}_{\mathrm{n}}
$$

yields for $\delta=1 /\left(1+2 \mathrm{C}_{\mathrm{n}}\right)>0$ and $u=1 / \psi(\delta \mathrm{t})$ that

$$
\int_{c_{\star}}^{u^{2}} \frac{N(\mathrm{~s})}{\mathrm{s}} d \mathrm{~s} \leq\left(1+2 \mathrm{C}_{\mathrm{n}}\right) \int_{c_{\star}}^{u} \frac{N(\mathrm{~s})}{\mathrm{s}} d \mathrm{~s}
$$

or equivalently, that $\psi(\mathrm{t}) \leq \psi(\delta \mathrm{t})^{2}$. The bound (2.15) applies for $N(\mathrm{~s})=A(\log (1+\mathrm{s}))^{\beta}$ with $\beta>0$, yielding the stretched exponential decay $\psi(\mathrm{t})=A^{\prime} \exp \left(-b \mathrm{t}^{1 /(1+\beta)}\right)$ (see [BPS] and the references therein for more details about this type of Nash profiles). 
Unlike the setting of Remark 2.6, for our main focus the Nash profile $N(\cdot)$ induced by the doubling function $v(r)$ has at least polynomial growth, namely satisfies (2.10), so we improve the bound (2.14) by adapting next the argument of [SZ2, proof of Theorem 2.3].

Lemma 2.7. In the setting of Lemma 2.5 if $A_{n} \geq 0$ are such that for $n \in[m, N]$

$$
A_{n}^{2} \leq \sup _{m \leq \ell \leq n}\left\{\frac{A_{\ell}}{\psi_{n}(n-\ell)}\right\}
$$

then

$$
M_{N}:=\sup _{n \in[m, N]}\left\{A_{n}\left\|K_{m, n}\right\|_{L^{1}\left(\nu_{n}\right) \rightarrow L^{\infty}\left(\nu_{m}\right)}\right\} \leq 1 .
$$

Proof. Considering (2.11) at some $\ell \in[m, n]$ we have by Lemma 2.5 that

$$
\begin{aligned}
\left\|K_{m, n}^{\star}\right\|_{L^{1}\left(\nu_{m}\right) \rightarrow L^{\infty}\left(\nu_{n}\right)} & \leq\left\|K_{m, \ell}^{\star}\right\|_{L^{1}\left(\nu_{m}\right) \rightarrow L^{2}\left(\nu_{\ell}\right)}\left\|K_{\ell, n}^{\star}\right\|_{L^{2}\left(\nu_{\ell}\right) \rightarrow L^{\infty}\left(\nu_{n}\right)} \\
& \leq\left\|K_{m, \ell}^{\star}\right\|_{L^{1}\left(\nu_{m}\right) \rightarrow L^{2}\left(\nu_{\ell}\right)} \psi_{n}(n-\ell)^{\frac{1}{2}} .
\end{aligned}
$$

Recall that a Markov kernel $K_{m, \ell}$ is a contraction from $L^{\infty}\left(\nu_{\ell}\right)$ to $L^{\infty}\left(\nu_{m}\right)$ (as $\nu_{\ell}$ is strictly positive on $V$ ). By duality, the adjoint $K_{m, \ell}^{\star}$ is thus a contraction from $L^{1}\left(\nu_{m}\right)$ to $L^{1}\left(\nu_{\ell}\right)$. Moreover, for any $\ell$ and $f_{\ell}$

$$
\left\|f_{\ell}\right\|_{L^{2}\left(\nu_{\ell}\right)}^{2} \leq\left\|f_{\ell}\right\|_{L^{\infty}\left(\nu_{\ell}\right)}\left\|f_{\ell}\right\|_{L^{1}\left(\nu_{\ell}\right)}
$$

and taking $f_{\ell}=K_{m, \ell}^{\star} g$ for arbitrary $g \in L^{1}\left(\nu_{m}\right)$ we get that

$$
\left\|K_{m, \ell}^{\star}\right\|_{L^{1}\left(\nu_{m}\right) \rightarrow L^{2}\left(\nu_{\ell}\right)}^{2} \leq\left\|K_{m, \ell}^{\star}\right\|_{L^{1}\left(\nu_{m}\right) \rightarrow L^{\infty}\left(\nu_{\ell}\right)}\left\|K_{m, \ell}^{\star}\right\|_{L^{1}\left(\nu_{m}\right) \rightarrow L^{1}\left(\nu_{\ell}\right)} \leq\left\|K_{m, \ell}^{\star}\right\|_{L^{1}\left(\nu_{m}\right) \rightarrow L^{\infty}\left(\nu_{\ell}\right)} .
$$

By the definition of $M_{N}$, we thus deduce upon choosing the optimal $\ell$ from (2.16), that for any $n \in[m, N]$,

$$
\begin{aligned}
A_{n}^{2}\left\|K_{m, n}^{\star}\right\|_{L^{1}\left(\nu_{m}\right) \rightarrow L^{\infty}\left(\nu_{n}\right)}^{2} & \leq A_{n}^{2}\left\|K_{m, \ell}^{\star}\right\|_{L^{1}\left(\nu_{m}\right) \rightarrow L^{\infty}\left(\nu_{\ell}\right)} \psi_{n}(n-\ell) \\
& \leq A_{n}^{2} M_{N} A_{\ell}^{-1} \psi_{n}(n-\ell) \leq M_{N} .
\end{aligned}
$$

Finally, taking the supremum over $n$ in the LHS, we find that $M_{N} \leq 1$, as claimed.

Starting with an upper bound $N_{k}(\mathrm{~s})$ on the Nash profile functions, one merely applies Lemmas 2.5 and 2.7. That is, first solving the map (2.12) corresponding to $N_{k}(\mathrm{~s})$ in order to get an upper bound on $\psi_{n}(\cdot)$, from which by (2.16) one deduces the diagonal upper bound $1 / A_{n}$. This is much simplified in the presence of uniform bounds, as in Proposition 2.4.

Proof of Proposition 2.4. Considering Lemma 2.5 for $N_{k}(\cdot)=N(\cdot)$ and $\psi_{n}(0)=1 / c_{\star}$, we have the bound (2.11) for the non-increasing $\psi_{n}(t)=\psi(t)=1 / F^{-1}\left(t ; c_{\star}, N(\cdot)\right)$. We claim that

$$
C_{n}^{\prime}:=\sup _{n \geq 0}\left\{\frac{\psi(n / 4)}{\psi(n / 3)}\right\}^{2}<\infty
$$

in which case $A_{n}=1 /\left(\mathrm{C}_{\mathrm{n}}^{\prime} \psi(n / 3)\right)$ and $\ell=[3 n / 4]$ satisfy (2.16), with Lemma 2.7 completing the proof of the proposition. Next, setting $C_{n}^{\prime}=C^{2}$ and $u=1 / \psi(n / 4)$, we get (2.17) upon showing that $1 / \psi(n / 3) \leq C u$, or alternatively that for any $u \geq c_{\star}$

$$
h(u):=3 \int_{u}^{C u} \frac{N(\mathrm{~s})}{\mathrm{s}} d \mathrm{~s}-\int_{c_{\star}}^{u} \frac{N(\mathrm{~s})}{\mathrm{s}} d \mathrm{~s} \geq 0 .
$$


To this end, recall (2.10) that for $C \geq \mathrm{C}_{\mathrm{n}}>1$ and $u \geq \mathrm{s}_{0}$ we assumed that $N(C u) \geq 2 N(u)$, hence

$$
h^{\prime}(u)=\frac{3 N(C u)}{u}-\frac{4 N(u)}{u} \geq \frac{2 N(u)}{u} \geq 0 .
$$

Finally, with $u \mapsto N(u)$ non-decreasing, $h(u) \geq N(u)\left(3 \log C-\log \left(u / c_{\star}\right)\right)$ is non-negative for any $C \geq\left(\mathrm{s}_{0} / c_{\star}\right)^{1 / 3}$ and all $u \in\left[c_{\star}, s_{0}\right]$.

Coupling the dynamics of (1.3) and (1.5) we deduce part (b) out of part (a) of Theorem 1.4.

Proof of Theorem 1.4(b). From the arrival times $\left\{T_{n}^{\prime}\right\}$ of an auxiliary Poisson process of rate 2, we construct $\left\{Y_{t}\right\}$ obeying (1.5) by independently censoring the jump at each time $T_{n}^{\prime}$ with probability $1 / 2$ and proceeding at the non-censored $\left\{T_{n}\right\} \subseteq\left\{T_{n}^{\prime}\right\}$ as in (1.4). Fixing a realization $\omega=\left\{T_{n}^{\prime}\right\}$, at most $N_{t}-N_{s}$ jump attempts are made in $[s, t]$ by the corresponding dynamics $\left\{X_{n}\right\}$ of (1.3) having the $1 / 2$-uniformly lazy transitions $K_{n}^{(\omega)}:=\frac{1}{2} K_{T_{n}^{\prime}}+\frac{1}{2} I$. Recall that the Dirichlet form of any $\pi$-reversible $K$ has the symmetric form

$$
\mathcal{E}_{K, \pi}(f, g)=\frac{1}{2} \sum_{x, y \in V}(f(x)-f(y))(g(x)-g(y)) \pi(x) K(x, y)
$$

and under (1.12) we have that $Q(x, y) \geq \alpha_{l} K(x, y)$ for $Q=K^{2}$ and all $x, y \in V$. Thus, in the reversible case $\mathcal{E}_{K^{2}, \pi}(f, f) \geq \alpha_{l} \mathcal{E}_{K, \pi}(f, f)$ for all $f$ and consequently,

$$
\mathcal{N}_{K, \pi}(\mathrm{s}) \geq \alpha_{l} \mathcal{N}_{K^{2}, \pi}(\mathrm{s}), \quad \forall \mathrm{s} .
$$

This applies for $K=K_{n}^{(\omega)}, \alpha_{l}=1 / 2$ and the non-decreasing $n \mapsto \pi_{T_{n}^{\prime}}(x)$, hence by part (a) of Theorem 1.4 the bound (1.20) holds for $\left\{X_{n}\right\}$ with $\psi(\cdot)$ as stated in part (b). That is,

$$
\frac{K_{N_{s}, N_{t}}^{(\omega)}(x, y)}{\pi_{t}(y)} \leq \frac{K_{N_{s}, N_{t}}^{(\omega)}(x, y)}{\pi_{T_{N_{t}}^{\prime}}(y)} \leq \mathrm{C}_{\mathrm{n}}^{\prime} \psi\left(\frac{N_{t}-N_{s}}{3}\right) .
$$

To complete the proof, note that $K_{s, t}(x, y)$ is the expected value of $K_{N_{s}, N_{t}}^{(\omega)}(x, y)$ over $\omega$, whereas $N_{t}-N_{s} \sim \operatorname{Poisson}(2(t-s))$.

For the remainder of this section, we detail three additional situations in which good upper bounds on the Nash profiles are available. First, recalling that the Poincaré inequality together with uniform volume growth for $v(r)$ doubling, provide an upper bound on the Nash profile, in the following lemma we deduce from Theorem 1.4 that in the context of Theorem 1.8 the on-diagonal upper bound (1.20) holds for $\psi(\mathrm{t})=C^{\prime} / v(\sqrt{\mathrm{t}})$.

Lemma 2.8. Suppose $\mathbb{G}$ and non-decreasing $t \mapsto \pi_{t}(x)$ satisfy uniform volume growth $v(r)$ as in (1.11), for $v(r)$ doubling. Further, suppose the $\pi_{t}$-reversible $K_{t}$ satisfy the uniform PI (1.10), and in case of (1.3) such $\left\{K_{t}\right\}$ are also $\alpha_{l}$-uniformly lazy. Then, for $C^{\prime}\left(C_{\mathrm{P}}, \mathrm{C}_{\mathrm{v}}, \alpha_{l}\right)$ finite,

$$
\left\|K_{s, t}\right\|_{L^{1}\left(\pi_{t}\right) \rightarrow L^{\infty}\left(\pi_{s}\right)}=\sup _{x, y \in V}\left\{\frac{K_{s, t}(x, y)}{\pi_{t}(y)}\right\} \leq \frac{C^{\prime}}{v(\sqrt{t-s})} \quad \forall s \in[0, t] .
$$

Proof. In case of the dynamic (1.3), with (2.19) applicable for $K_{t}$, by Theorem 1.4(a) it suffices to show that if $\pi$-reversible $K$ satisfies the Poincaré inequality (1.10) and the uniform growth assumption (1.11) for volume doubling $v(r)$, then for $\beta=2, v^{-1}(\mathrm{~s}):=\inf \{r \geq 1: v(r) \geq \mathrm{s}\}$ and some $C\left(\mathrm{C}_{\mathrm{P}}, \mathrm{C}_{\mathrm{v}}\right)$ finite,

$$
\alpha_{l} N(\mathrm{~s}):=C\left(v^{-1}(C \mathrm{~s})\right)^{\beta} \geq \mathcal{N}_{K, \pi}(\mathrm{s}), \quad \forall \mathrm{s} .
$$


Indeed, $v^{-1}\left(\mathrm{~s} / \mathrm{C}_{\mathrm{v}}^{k}\right) \leq 2^{-k} v^{-1}(\mathrm{~s})$ so $N\left(\mathrm{C}_{\mathrm{v}} \mathrm{s}\right) \geq 4 N(\mathrm{~s})$, namely (2.10) holds with $\mathrm{C}_{\mathrm{n}}=\mathrm{C}_{\mathrm{v}}$. Moreover, splitting the integral (1.21) for such non-decreasing $\mathrm{s} \mapsto N(\mathrm{~s})$, to intervals $\left[u / \mathrm{C}_{\mathrm{v}}^{k+1}, u / \mathrm{C}_{\mathrm{v}}^{k}\right], k \geq 0$ shows that the RHS of (1.21) is dominated by the largest intervals and hence for some $C^{\prime}=$ $C^{\prime}\left(C / \alpha_{l}, \mathrm{C}_{\mathrm{v}}\right)$ finite and any $\mathrm{t} \geq 1 / 3$,

$$
\psi(\mathrm{t})=\frac{1}{F^{-1}\left(\mathrm{t} ; \underline{\pi_{0}}, N(\cdot)\right)} \leq \frac{C^{\prime}}{v\left((3 \mathrm{t})^{1 / \beta}\right)} .
$$

Thus, multiplying $C^{\prime}$ by $C_{n}^{\prime}$ we get (2.20) as a consequence of (1.20). Further, recall Theorem 1.4 (b) that for the dynamic (1.5) the preceding bound on $\psi(\mathrm{t})$ always hold (with $\alpha_{l}=1 / 2$ ), and in this context we arrive for $N_{\lambda} \sim \operatorname{Poisson}(\lambda)$ and the non-decreasing $v(r) \geq 1$, at

$$
\left\|K_{s, t}\right\|_{L^{1}\left(\pi_{t}\right) \rightarrow L^{\infty}\left(\pi_{s}\right)} \leq \mathbb{E}\left[\frac{C^{\prime}}{v\left(\left(N_{2(t-s)}\right)^{1 / \beta}\right)}\right] \leq \frac{C^{\prime}}{v\left((t-s)^{1 / \beta}\right)}+C^{\prime} e^{-(t-s) / \kappa}
$$

(as $\mathbb{P}\left(N_{2 \lambda} \leq \lambda\right) \leq e^{-\lambda / \kappa}$ for some $\kappa$ finite and all $\lambda \geq 0$ ). With $v(r) e^{-r^{2} / \kappa}$ uniformly bounded, upon increasing $C^{\prime}$ we thus get (2.20) out of (2.23).

Turning to establish (2.21), recall that from (2.18) and the covering argument in the proof of [SC, Lemma 2.4], it follows that for some $c=c\left(\mathrm{C}_{\mathrm{P}}, \mathrm{C}_{\mathrm{v}}\right)$ finite, any $f \in L^{2}(\pi)$ and $r>0$

$$
\left\|f-f_{r}\right\|_{L^{2}(\pi)}^{2} \leq c r^{2} \mathcal{E}_{K, \pi}(f, f),
$$

where

$$
f_{r}(x):=\frac{1}{\pi(\mathbb{B}(x, r))} \sum_{y \in \mathbb{B}(x, r)} f(y) \pi(y) .
$$

Further, by the uniform volume growth assumption of (1.11),

$$
\begin{aligned}
\left\|f_{r}\right\|_{L^{\infty}(\pi)} & \leq \frac{1}{\inf _{x} \pi(\mathbb{B}(x, r))}\|f\|_{L^{1}(\pi)} \leq \frac{C_{v}}{v(r)}\|f\|_{L^{1}(\pi)}, \\
\left\|f_{r}\right\|_{L^{1}(\pi)} & \leq C_{\mathrm{v}}^{2}\|f\|_{L^{1}(\pi)} .
\end{aligned}
$$

Consequently

$$
\|f\|_{L^{2}(\pi)}^{2} \leq 2\left\|f-f_{r}\right\|_{L^{2}(\pi)}^{2}+2\left\|f_{r}\right\|_{L^{2}(\pi)}^{2} \leq 2 c r^{2} \mathcal{E}_{K, \pi}(f, f)+\frac{2 \mathrm{C}_{\mathrm{v}}^{3}}{v(r)}\|f\|_{L^{1}(\pi)}^{2} .
$$

Setting $C:=2 c+2 \mathrm{C}_{\mathrm{v}}^{3}$ and $r=v^{-1}(C \mathbf{s})$, it follows from (2.24) that if $\|f\|_{L^{1}(\pi)}^{2} \leq \mathrm{s}$ and $\|f\|_{L^{2}(\pi)}^{2}=1$, then

$$
\frac{1}{\mathcal{E}_{K, \pi}(f, f)} \leq C r^{2}=\alpha_{l} N(\mathrm{~s}) .
$$

To complete the proof, recall that $\mathcal{N}_{K, \pi}(\mathrm{s})$ is the maximum of $1 / \mathcal{E}_{K, \pi}(f, f)$ over such $f$.

One can also obtain upper bounds on the Nash profiles from lower bounds on isoperimetric profiles of the Markov operators. Indeed, there is a tight connection between the Nash and isoperimetric profiles of a Markov chain $Q$ having invariant measure $\pi$. Specifically, recall the $L^{2}$-isoperimetric (or spectral) profile of such a chain $(Q, \pi)$ on infinite state space $V$, defined as the non-increasing

$$
\Lambda_{Q, \pi}(u)=\inf \left\{\lambda_{Q}(\Omega): \Omega \subseteq V, \pi(\Omega) \leq u\right\}
$$

where

$$
\lambda_{Q}(\Omega)=\inf \left\{\mathcal{E}_{Q, \pi}(f, f): \operatorname{support}(f) \subseteq \Omega,\|f\|_{L^{2}(\pi)}=1\right\}
$$


In words, $\lambda_{Q}(\Omega)$ is the smallest eigenvalue of the operator $I-Q$ with Dirichlet boundary condition in $\Omega$. Also recall the $L^{1}$-isoperimetric (or conductance) profile

$$
\Phi_{Q, \pi}(u)=\inf \left\{\frac{1}{\pi(\Omega)} \sum_{x \in \Omega} \pi(x) Q\left(x, \Omega^{c}\right): \Omega \subseteq V, \pi(\Omega) \leq u\right\} .
$$

The $L^{2}$ and $L^{1}$ profiles are related via Cheeger's inequality (see [LS]),

$$
\frac{1}{2} \Phi_{Q, \pi}^{2}(u) \leq \Lambda_{Q, \pi}(u) \leq \Phi_{Q, \pi}(u) .
$$

As shown next, the Nash profile $\mathcal{N}_{Q, \pi}(\cdot)$ contains the same information as the $L^{2}$-isoperimetric profile (see GMT, Lemma 2.1] for such a result in case of finite Markov chains; the proof for $V$ infinite is provided here for the reader's convenience).

Lemma 2.9. For Markov operator $Q$, its $\sigma$-finite invariant measure $\pi$, and any $u>0$,

$$
\frac{1}{\Lambda_{Q, \pi}(u)} \leq \mathcal{N}_{Q, \pi}(u) \leq \frac{2}{\Lambda_{Q, \pi}(4 u)}
$$

Proof. By the Cauchy-Schwarz inequality $\|f\|_{L^{1}(\pi)}^{2} \leq \pi(\Omega)\|f\|_{L^{2}(\pi)}^{2}$ for any $f$ supported within $\Omega$, yielding that $\Lambda_{Q, \pi}(u) \geq 1 / \mathcal{N}_{Q, \pi}(u)$ via the definitions (1.18) and (2.25). We proceed to show that $\Lambda_{Q, \pi}(4 u) \leq 2 / \mathcal{N}_{Q, \pi}(u)$. Namely, that for any $f \in L^{2}(\pi)$

$$
\|f\|_{L^{1}(\pi)}^{2}=u\|f\|_{L^{2}(\pi)}^{2} \quad \Longrightarrow \quad \frac{1}{2}\|f\|_{L^{2}(\pi)}^{2} \Lambda_{Q, \pi}(4 u) \leq \mathcal{E}_{Q, \pi}(f, f) .
$$

Indeed, recall from (2.25) that for $f \in L^{2}(\pi)$,

$$
\|f\|_{L^{2}(\pi)}^{2} \Lambda_{Q, \pi}(\pi(\operatorname{support}(f))) \leq \mathcal{E}_{Q, \pi}(f, f) .
$$

Next, for $\mathrm{t} \geq 0$ set $f_{\mathrm{t}}:=(f-\mathrm{t})_{+}$supported on $\Omega_{\mathrm{t}}:=\{f>\mathrm{t}\}$. Obviously $f_{\mathrm{t}} \in L^{2}(\pi)$, with $f^{2}-2 \mathrm{t}|f| \leq f_{\mathrm{t}}^{2}$ and $\mathcal{E}_{Q, \pi}\left(f_{\mathrm{t}}, f_{\mathrm{t}}\right) \leq \mathcal{E}_{Q, \pi}(f, f)$. Hence,

$$
\left[\|f\|_{L^{2}(\pi)}^{2}-2 \mathrm{t}\|f\|_{L^{1}(\pi)}\right] \Lambda_{Q, \pi}\left(\pi\left(\Omega_{\mathrm{t}}\right)\right) \leq\left\|f_{\mathrm{t}}\right\|_{L^{2}(\pi)}^{2} \Lambda_{Q, \pi}\left(\pi\left(\Omega_{\mathrm{t}}\right)\right) \leq \mathcal{E}_{Q, \pi}\left(f_{\mathrm{t}}, f_{\mathrm{t}}\right) \leq \mathcal{E}_{Q, \pi}(f, f) .
$$

Since $\pi\left(\Omega_{\mathrm{t}}\right) \leq \mathrm{t}^{-1}\|f\|_{L^{1}(\pi)}$, if $4 \mathrm{t}\|f\|_{L^{1}(\pi)}=\|f\|_{L^{2}(\pi)}^{2}$ for $\mathrm{t}=\|f\|_{L^{1}(\pi)} /(4 u)$ finite, then

$$
\frac{1}{2}\|f\|_{L^{2}(\pi)}^{2} \Lambda_{Q, \pi}(4 u) \leq \frac{1}{2}\|f\|_{L^{2}(\pi)}^{2} \Lambda_{Q, \pi}\left(\pi\left(\Omega_{\mathrm{t}}\right)\right) \leq \mathcal{E}_{Q, \pi}(f, f),
$$

as claimed in (2.28).

By Lemma 2.9, any lower bound on the $L^{2}$ or $L^{1}$-isoperimetric profile can be turned into an upper bound on the Nash profile.

Example 2.10. Consider Example 2.3 with $\nu_{n}=\pi_{n}$ invariant for $K_{n}$ such that $n \mapsto \pi_{n}(x)$ are non-decreasing (and $Q_{n}=K_{n}^{\star} K_{n}$ ). Suppose also that for some $d>0$ and positive $\kappa_{n}$

$$
\mathcal{N}_{Q_{n}, \pi_{n}}(\mathrm{~s}) \leq 4 \alpha^{-1} \kappa_{n}^{-2}(4 \mathrm{~s})^{2 / d}, \quad \forall \mathrm{s}, n,
$$

where $\gamma_{n}:=\sum_{m=1}^{n} \kappa_{m}^{2}$ are such that for some $c_{0} \geq 2$,

$$
\gamma_{n} \geq c_{0} \quad \Longrightarrow \quad \exists \ell(n) \quad \frac{1}{3} \leq \frac{1+\gamma_{\ell(n)}}{1+\gamma_{n}} \leq \frac{2}{3} .
$$

It is easy to check that for some $c=c\left(\alpha, d, \underline{\pi_{0}}\right)$ finite,

$$
\psi_{n}(j)=c\left(1+\gamma_{n}-\gamma_{n-j}\right)^{-d / 2},
$$


satisfies (2.12) and consequently the bound (2.11). The condition (2.30) allows for taking $A_{n}=$ $c_{1}^{-1}\left(1+\gamma_{n}\right)^{d / 2}$ with $c_{1}=c\left(1+c_{0}\right)^{d}$ in Lemma 2.7, thereby concluding that

$$
\sup _{x, y \in V}\left\{\frac{K_{0, n}(x, y)}{\pi_{n}(y)}\right\} \leq c_{1}\left(1+\sum_{m=1}^{n} \kappa_{m}^{2}\right)^{-d / 2} .
$$

In particular, the bound (2.31) recovers DHMP, Theorem 1.2], proved before for the dynamic (1.3) with $\left(K_{t}, \pi_{t}\right)$ of (1.1)-(1.2) via evolving sets techniques. More precisely, DHMP assume that the uniform lazy property (1.12) holds and $L^{1}$-isoperimetric profiles such that for some $d>1$ and positive $\kappa_{n}$

$$
\Phi_{K_{n}, \pi_{n}}(\mathrm{~s}) \geq \kappa_{n} \mathrm{~s}^{-1 / d} .
$$

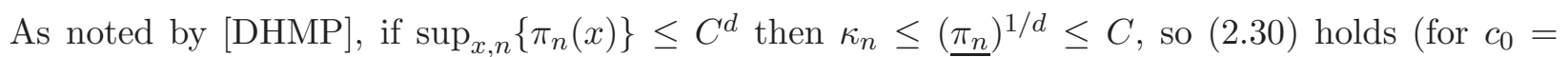
$\left.2+3 C^{2}\right)$. Recall the left inequality of (2.21) that for $Q_{n}=\bar{K}_{n}^{2}$ in the reversible case

$$
\mathcal{N}_{Q_{n}, \pi_{n}}(\mathrm{~s}) \leq \alpha_{l}^{-1} \mathcal{N}_{K_{n}, \pi_{n}}(\mathrm{~s}), \quad \forall \mathrm{s}, n .
$$

Finally, by Lemma 2.9 and Cheeger's inequality (2.27), the isoperimetric bound (2.32) implies

$$
\mathcal{N}_{K_{n}, \pi_{n}}(\mathrm{~s}) \leq 2 \Lambda_{K_{n}, \pi_{n}}(4 \mathrm{~s})^{-1} \leq 4 \Phi_{K_{n}, \pi_{n}}(4 \mathrm{~s})^{-2} \leq 4 \kappa_{n}^{-2}(4 \mathrm{~s})^{2 / d}, \quad \forall \mathrm{s}, n .
$$

So, the assumptions of [DHMP] imply both (2.29) and (2.30), thereby yielding (2.31).

Theorem 1.4 and Proposition 2.4 can be applied to general Markov operators $K_{t}$, in particular, having non-local jumping kernels. The following example illustrates this point.

Example 2.11. Let $d(x, y)$ denote the graph distance on a locally finite, connected, infinite, nonoriented graph $\mathbb{G}=(V, E)$. Suppose that for each $n \in \mathbb{N}$ the non-local Markov kernel $K_{n}$ is reversible with respect to the measure $\pi_{n}$, such that:

- For every $x \in V$ the sequence $n \mapsto \pi_{n}(x)$ is non-decreasing, and $\pi_{n}$ satisfies uniform volume growth with $v(r)$ doubling as in (1.11).

- There exist $A<\infty$ and $\beta \in(0,2)$ such that for any $x \neq y \in V$,

$$
\frac{K_{n}(x, y)}{\pi_{n}(y)} \geq \frac{d(x, y)^{-\beta}}{A v(d(x, y))}
$$

It is easy to see that upon changing $A$ to $A^{2} v(2) 2^{\beta} \mathrm{C}_{\mathrm{v}}$, the bound (2.33) applies also for $K_{n}^{2}(x, y)$. Hence, by [CK, Theorem 3.1] there exist finite $c_{i}=c_{i}\left(\mathrm{C}_{\mathrm{v}}, A, \beta\right), i=1,2$, such that for $\theta(r):=$ $r\left(v^{-1}\left(c_{1} / r\right)\right)^{-\beta}$ and the inverse $v^{-1}$ of the function $r \mapsto v(r)$, the following Nash inequality holds for every $f \in \operatorname{Dom}\left(\mathcal{E}_{K_{n}^{2}, \pi_{n}}\right)$ with $\|f\|_{L^{1}\left(\pi_{n}\right)}=1$ :

$$
\theta\left(\|f\|_{L^{2}\left(\pi_{n}\right)}^{2}\right) \leq c_{2} \mathcal{E}_{K_{n}^{2}, \pi_{n}}(f, f) .
$$

Equivalently, the Nash profile of $\left(K_{n}^{2}, \pi_{n}\right)$ then satisfies

$$
N(\mathrm{t}):=c_{2}\left(v^{-1}\left(c_{1} \mathrm{t}\right)\right)^{\beta} \geq \mathcal{N}_{K_{n}^{2}, \pi_{n}}(\mathrm{t}) .
$$

As in the derivation of (2.22), it then follows from Theorem 1.4 (now with $C_{n}=C_{v}^{\lceil 1 / \beta\rceil}$ ), that for the dynamics (1.3), some finite $c_{3}=c_{3}\left(\mathrm{C}_{\mathrm{v}}, A, \beta\right)$ and all integers $s<t$,

$$
\sup _{x, y \in V}\left\{\frac{K_{s, t}(x, y)}{\pi_{t}(y)}\right\} \leq \frac{c_{3}}{v\left((t-s)^{1 / \beta}\right)}
$$




\section{Gaussian upper bounds}

We adapt the technique of [HS, Section 2] for deriving off-diagonal Gaussian upper bounds via complex interpolation techniques. Specifically, in this section we work with $L^{p}$ spaces of $\mathbb{C}$-valued functions, with $\mathcal{C}_{0}(V)$ denoting the dense linear subspace of finitely supported $\mathbb{C}$-valued functions. Considering $\rho: V \rightarrow \mathbb{R}$ such that the non-negative linear operators on $\mathcal{C}_{0}(V)$

$$
K_{s, t}^{\theta} f(x):=w_{-\theta} K_{s, t}\left(w_{\theta} f\right)(x), \quad w_{\theta}(x):=e^{\theta \rho(x)}, \quad 0 \leq s \leq t \leq T, \quad \theta \in \mathbb{R},
$$

have bounded $L^{2}\left(\nu_{t}\right) \rightarrow L^{2}\left(\nu_{s}\right)$ norms, we study the unique continuous extension of $K_{s, t}^{\theta}$ for both continuous and discrete time (where $s, t \in \mathbb{Z}_{+}$). Our main example is $\rho(x)=d\left(x, x_{0}\right)$ for the graph distance $d(x, y)=d_{\mathbb{G}}(x, y)$ in a locally finite, connected graph $\mathbb{G}$ and a fixed vertex $x_{0} \in V$.

For completeness we first prove the following proposition, which summarizes the interpolation method of [HS].

Proposition 3.1. (see [HS, Lemma 2.2]) Suppose $\left\{K_{s, t}^{\theta}\right\}$ are as in (3.1), with non-negative linear operators $K_{s, t}=K_{s, \xi} K_{\xi, t}, s \leq \xi \leq t$ (with $K_{\xi, \xi}=I$ ), such that

$$
\left\|K_{s, t}\right\|_{L^{\infty}\left(\nu_{t}\right) \rightarrow L^{\infty}\left(\nu_{s}\right)} \leq 1, \quad \forall 0 \leq s \leq t \leq T,
$$

for strictly positive $\sigma$-finite measures $\left\{\nu_{s}\right\}$, with $\nu_{0} \in \mathcal{M}_{+}(V)$. Suppose that for $t \geq s \in[0, T]$ :

(a) For a non-decreasing $t \mapsto a_{t}$ satisfying (1.30), the Gaffney bound

$$
\begin{aligned}
\left\|K_{s, t}^{\theta}\right\|_{L^{2}\left(\nu_{t}\right) \rightarrow L^{2}\left(\nu_{s}\right)} & \leq \exp \left(a_{t}-a_{s}+\chi(\theta)(t-s)\right), & & \forall \theta \in \mathbb{R}, \\
\text { with } \quad c_{1}^{-1} \theta^{2} & \leq \chi(\theta) \leq c_{1} \theta^{2}, & & \forall|\theta| \leq \delta_{\star},
\end{aligned}
$$

holds on $\mathcal{C}_{0}(V)$, for $|\theta| \mapsto \chi(\theta)$ non-decreasing, some $\delta_{\star}>0$ and $c_{1}<\infty$.

(b) The $2 \rightarrow \infty$ bound

$$
\left\|K_{s, t}\right\|_{L^{2}\left(\nu_{t}\right) \rightarrow L^{\infty}\left(\nu_{s}\right)} \leq \varphi(t-s),
$$

holds with $\tau \mapsto \varphi(\tau) \tau^{\beta}$ non-decreasing on $[0, T]$ for some $\beta>0$.

Then, for some finite $C_{1}\left(c_{1}, \beta, \delta_{\star}\right)$ and $C_{2}=C_{2}\left(A, \beta, \underline{\nu_{0}}, \varphi(1)\right)$,

$$
\left\|K_{0, T}^{\theta}\right\|_{L^{2}\left(\nu_{T}\right) \rightarrow L^{\infty}\left(\nu_{0}\right)} \leq C_{2} \varphi(T) \exp \left(C_{1} \chi(\theta) T\right), \quad \forall \theta \in \mathbb{R}, \quad T \geq 1
$$

Remark 3.2. Clearly, (3.2) holds for Markov transition probabilities $\left\{K_{s, t}\right\}$ and strictly positive $\left\{\nu_{s}\right\}$.

Proof. Since $(\underline{\nu})^{1 / q}\|f\|_{L^{\infty}(\nu)} \leq\|f\|_{L^{q}(\nu)}$ for any $f$ and $\nu \in \mathcal{M}_{+}(V)$, necessarily

$$
\left\|K_{0, t}^{\theta}\right\|_{L^{2 p}\left(\nu_{t}\right) \rightarrow L^{\infty}\left(\nu_{0}\right)} \leq\left(\underline{\nu_{0}}\right)^{-1 /(2 q)}\left\|K_{0, t}^{\theta}\right\|_{L^{2 p}\left(\nu_{t}\right) \rightarrow L^{2 q}\left(\nu_{0}\right)}, \quad \forall p, q, t>0 .
$$

Considering (3.3) and (3.7) at $s=0, t=T, p=q=1$, our assumption (3.4) and having $\varphi(T) \geq$ $\varphi(1) T^{-\beta}$ for $T \geq 1$, yield (3.6) for $C_{1}=1+c_{1} \kappa \beta / \delta_{\star}^{2}, C_{2}=\left(\underline{\nu_{0}}\right)^{-1 / 2} \varphi(1)^{-1}$ and $|\theta| \geq \delta_{\star} /(1+\log T)$, where

$$
\kappa:=\sup _{t \geq 1}\left\{\frac{\log t}{t}(1+\log t)^{2}\right\}<\infty .
$$

We proceed to derive (3.6) when $|\theta|<\delta_{\star} /(1+\log T)$ by closely following [SC2, Subsection 4.2.2]. To this end, (3.2) and (3.5) are invariant under the re-scaling $\nu_{s ; t}:=e^{-2\left(a_{t}-a_{s}\right)} \nu_{s}$ and yield by Riesz-Thorin interpolation theorem that

$$
\left\|K_{s, t}\right\|_{L^{2 p}\left(\nu_{t}\right) \rightarrow L^{\infty}\left(\nu_{s ; t}\right)} \leq \varphi(t-s)^{1 / p}, \quad \forall p \geq 1 .
$$


To apply Stein's interpolation theorem, consider the $\mathbb{C}$-valued weights $w_{\theta z}(x)=w_{\theta}(x)^{z}$ indexed on the strip $\mathbb{S}:=\{z=u+i b: u=\Re(z) \in[0,1]\}$. For fixed $\theta \in \mathbb{R}$ and $m \leq \ell$ the associated map $z \mapsto K_{m, \ell}^{\theta z}=w_{-\theta z} K_{m, \ell} w_{\theta z}$ forms an $\mathbb{S}$-analytic collection of linear operators on $\mathcal{C}_{0}(V)$ such that $K_{m, \ell}^{\theta(u+i b)}=w_{-i \theta b} K_{m, \ell}^{\theta u} w_{i \theta b}$. With $\left|w_{ \pm i \theta b}(x)\right|=1$ we thus have for $u=0$ the $L^{\infty}$-contraction

$$
\left\|K_{s, t}^{\theta i b}\right\|_{L^{\infty}\left(\nu_{t}\right) \rightarrow L^{\infty}\left(\nu_{s ; t}\right)} \leq\left\|K_{s, t}\right\|_{L^{\infty}\left(\nu_{t}\right) \rightarrow L^{\infty}\left(\nu_{s ; t}\right)} \leq 1
$$

Moving to $\nu_{s ; t}$ eliminates the term $a_{t}-a_{s}$ in (3.3), so we get for $u=1$ the $L^{2}$-norm bound

$$
\left\|K_{s, t}^{\theta(1+i b)}\right\|_{L^{2}\left(\nu_{t}\right) \rightarrow L^{2}\left(\nu_{s ; t}\right)} \leq\left\|K_{s, t}^{\theta}\right\|_{L^{2}\left(\nu_{t}\right) \rightarrow L^{2}\left(\nu_{s ; t}\right)} \leq \exp (\chi(\theta)(t-s)) .
$$

By Stein's interpolation (see [SW]), from (3.9) and (3.10) we have for $\lambda=1 / p \in[0,1]$,

$$
\left\|K_{s, t}^{\theta \lambda}\right\|_{L^{2 p}\left(\nu_{t}\right) \rightarrow L^{2 p}\left(\nu_{s ; t}\right)} \leq \exp (\lambda \chi(\theta)(t-s))
$$

and upon replacing $\theta$ by $\theta / \lambda$, deduce that for all $\theta \in \mathbb{R}$,

$$
\left\|K_{s, t}^{\theta(1+i b)}\right\|_{L^{2 p}\left(\nu_{t}\right) \rightarrow L^{2 p}\left(\nu_{s ; t}\right)} \leq\left\|K_{s, t}^{\theta}\right\|_{L^{2 p}\left(\nu_{t}\right) \rightarrow L^{2 p}\left(\nu_{s ; t}\right)} \leq \exp \left(p^{-1} \chi(\theta p)(t-s)\right) .
$$

Next, employing (3.8) gives that

$$
\left\|K_{s, t}^{\theta i b}\right\|_{L^{2 p}\left(\nu_{t}\right) \rightarrow L^{\infty}\left(\nu_{s ; t}\right)} \leq\left\|K_{s, t}\right\|_{L^{2 p}\left(\nu_{t}\right) \rightarrow L^{\infty}\left(\nu_{s ; t}\right)} \leq \varphi(t-s)^{1 / p} .
$$

With $\nu_{t}=\nu_{t ; t}$, from (3.11) and (3.12) we conclude by yet another application of Stein's interpolation theorem, that for $\lambda=p / q \in[0,1]$ and any $q \geq p \geq 1$,

$$
\left\|K_{s, t}^{\theta \lambda}\right\|_{L^{2 p}\left(\nu_{t ; t}\right) \rightarrow L^{2 q}\left(\nu_{s ; t}\right)} \leq \varphi(t-s)^{\frac{1-\lambda}{p}} \exp \left(\lambda p^{-1} \chi(\theta p)(t-s)\right) .
$$

Considering $\widehat{\nu}_{t}=\nu_{t ; T}$ and replacing once more $\theta$ by $\theta / \lambda$, we get that

$$
\begin{aligned}
\left\|K_{s, t}^{\theta}\right\|_{L^{2 p}\left(\widehat{\nu}_{t}\right) \rightarrow L^{2 q}\left(\widehat{\nu}_{s}\right)} & =e^{\left(a_{T}-a_{t}\right)(1 / p-1 / q)}\left\|K_{s, t}^{\theta}\right\|_{L^{2 p}\left(\nu_{t ;}\right) \rightarrow L^{2 q}\left(\nu_{s ; t}\right)} \\
& \leq\left(e^{a_{T}-a_{t}} \varphi(t-s)\right)^{(1 / p-1 / q)} \exp \left(q^{-1} \chi(\theta q)(t-s)\right) .
\end{aligned}
$$

Next, proceeding similarly to the proof of [HS, Lemma 2.2], set $\eta_{j}=c_{0} j^{-2}$ such that $\sum_{j \geq 2} \eta_{j}=1$ and partition $T$ into (non-increasing) blocks $\ell_{j}=\left\lfloor\eta_{j} T\right\rfloor \geq 1$ for $2 \leq j \leq m \leq \sqrt{c_{0} T}$ and $\ell_{1}=$ $T-\sum_{j=2}^{m} \ell_{j}$. We further set the corresponding strictly decreasing

$$
t_{j}=\sum_{k=j+1}^{m} \ell_{k}, \quad 0 \leq j \leq m .
$$

With $\underline{\widehat{\nu}_{0}}=e^{-2 a_{T}} \underline{\nu_{0}}$, in view of (3.7) we get for any non-decreasing $q_{j} \geq q_{0}=1$,

$$
\begin{aligned}
\left\|K_{0, T}^{\theta}\right\|_{L^{2}\left(\nu_{T}\right) \rightarrow L^{\infty}\left(\nu_{0}\right)} & =\left\|K_{0, T}^{\theta}\right\|_{L^{2}\left(\widehat{\nu}_{T}\right) \rightarrow L^{\infty}\left(\widehat{\nu}_{0}\right)} \\
& \leq \kappa_{0} e^{a_{T} / q_{m}} \prod_{j=1}^{m}\left\|K_{t_{j}, t_{j-1}}^{\theta}\right\|_{L^{2 q_{j-1}\left(\widehat{\nu}_{t_{j-1}}\right) \rightarrow L^{2 q_{j}}\left(\widehat{\nu}_{t_{j}}\right)}} \quad \forall \theta \in \mathbb{R},
\end{aligned}
$$

where $\kappa_{0}:=\left(1 \wedge \underline{\nu}_{0}\right)^{-1}$ is finite. Further, from (1.30) we have that for $A_{o}=A / \log 2$,

$$
a_{T}-a_{t} \leq A+A_{o} \log \left(\frac{T+1}{t+1}\right) \leq A+A_{o} \log (T / t), \quad \forall t \in[0, T] .
$$


Recall (3.14) that $t_{j-1} \geq \ell_{j}$, hence by our interpolation bound (3.13) and (3.16),

$$
\left\|K_{t_{j}, t_{j-1}}^{\theta}\right\|_{L^{2 q_{j-1}\left(\widehat{\nu}_{t_{j-1}}\right) \rightarrow L^{2 q_{j}}\left(\widehat{\nu}_{t_{j}}\right)}} \leq\left(e^{A}\left(T / \ell_{j}\right)^{A_{o}} \varphi\left(\ell_{j}\right)\right)^{\left(1 / q_{j-1}-1 / q_{j}\right)} \exp \left(q_{j}^{-1} \chi\left(\theta q_{j}\right) \ell_{j}\right) .
$$

Upon plugging these bounds into (3.15), recalling (3.4), that $a_{T} \leq 2 A+A_{o} \log T$ for $T \geq 1$, and our assumption that $\tau \mapsto \varphi(\tau) \tau^{\beta}$ is non-decreasing on $[0, T]$, we find that for $|\theta| \leq \delta_{\star} / q_{m}$ and $\beta_{\star}:=\beta+A_{o}$,

$$
\begin{aligned}
\left\|K_{0, T}^{\theta}\right\|_{L^{2}\left(\nu_{T}\right) \rightarrow L^{\infty}\left(\nu_{0}\right)} & \leq \kappa_{0} e^{2 A} T^{A_{0} / q_{m}} \prod_{j=1}^{m}\left(\left(T / \ell_{j}\right)^{A_{0}} \varphi\left(\ell_{j}\right)\right)^{\left(1 / q_{j-1}-1 / q_{j}\right)} \exp \left(c_{1} q_{j} \ell_{j} \theta^{2}\right) \\
& \leq \zeta_{T} \varphi(T) \exp \left(\beta_{\star} \gamma_{T}+c_{1}^{2} b_{T} \chi(\theta) T\right)
\end{aligned}
$$

where

$$
b_{T}:=\sum_{j=1}^{m} q_{j} \frac{\ell_{j}}{T}, \quad \gamma_{T}:=\sum_{j=1}^{m}\left(\frac{1}{q_{j-1}}-\frac{1}{q_{j}}\right) \log \left(T / \ell_{j}\right), \quad \zeta_{T}:=\kappa_{0} e^{2 A} \varphi(1)^{-1 / q_{m}} T^{\beta_{\star} / q_{m}} .
$$

Set $q_{j}:=1+(\log j)_{+}^{2}$ and maximal $m(T) \geq 1$ such that $q_{m}<1+\kappa_{1}^{-1} \log T$, with $\kappa_{1} \geq 2 / \log 2$ implying $c_{0} T \geq T \geq m^{2}$. It yields $q_{m} \geq 1$ such that $\log T / q_{m} \leq 2 \kappa_{1}$, hence $\zeta_{T}$ is uniformly bounded. Further, both $\gamma_{T}$ and $b_{T}$ are bounded by some universal constant since the series $\sum_{j}(\log j)\left(1 / q_{j-1}-1 / q_{j}\right)$ and $\sum_{j} q_{j} j^{-2}$ converge. Combined, these facts imply that (3.6) holds for some $C_{1}\left(c_{1}\right), C_{2}\left(A, \beta, \underline{\nu_{0}}, \varphi(1)\right)$ finite and all $|\theta|<\delta_{\star} /(1+\log T)$, as claimed.

Applying Proposition 3.1 yields the following heat-kernel off-diagonal estimate.

Proposition 3.3. Let $\psi(\cdot)$ be such that $\tau \mapsto \psi(\tau) \tau^{\beta}$ is non-decreasing for some $\beta>0$. Suppose Markov transition probabilities $\left\{K_{s, t}\right\}$ and strictly positive $\sigma$-finite measures $\left\{\nu_{s}\right\}$, with $\nu_{0} \in \mathcal{M}_{+}(V)$ such that $\left(\nu_{s} K_{s, t}\right) \leq \nu_{t}$ whenever $T \leq s \leq t \leq 2 T$, also satisfy the $1 \rightarrow \infty$ bound

$$
\left\|K_{s, t}\right\|_{L^{1}\left(\nu_{t}\right) \rightarrow L^{\infty}\left(\nu_{s}\right)} \leq \psi(t-s), \quad 0 \leq s \leq t \leq 2 T .
$$

Taking $\rho(\cdot)=d(\cdot, x)$, suppose the corresponding $\left\{K_{s, t}^{\theta}\right\}$ of (3.1) further satisfy the Gaffney bound (3.3)-(3.4) for all $t \geq s \in[0,2 T]$ (and some non-decreasing $t \mapsto a_{t}$ satisfying (1.30). Then, for some $C_{2}^{\prime}\left(A, \beta, \underline{\nu_{0}}, \psi(1)\right)$ finite and $\kappa \geq 4 C_{1}\left(c_{1}, \beta, \delta_{\star}\right)$,

$$
d(x, y) \leq \kappa \delta_{\star} T, T \geq 1 \Longrightarrow \frac{K_{0,2 T}(x, y)}{\nu_{2 T}(y)} \leq C_{2}^{\prime} \psi(T) \exp \left(-\frac{d^{2}(x, y)}{2 \kappa T}\right) .
$$

Proof. By Riesz-Thorin interpolation the $1 \rightarrow \infty$ bound (3.17) implies the $2 \rightarrow \infty$ bound (3.5) with $\varphi(\tau)=\psi(\tau)^{1 / 2}$. Hence, for $\rho(\cdot)=d(\cdot, x)$, we have from Proposition 3.1 that

$$
\left\|K_{0, T}^{\theta}\right\|_{L^{2}\left(\nu_{T}\right) \rightarrow L^{\infty}\left(\nu_{0}\right)} \leq C_{2} \psi(T)^{\frac{1}{2}} \exp \left(C_{1} \chi(\theta) T\right), \quad \forall T \geq 1, \quad \theta \in \mathbb{R} .
$$

Considering the adjoint $K_{s, t}^{\star}$ of $K_{s, t}: L^{2}\left(\nu_{t}\right) \rightarrow L^{2}\left(\nu_{s}\right)$, we have by duality that

$$
\left\|K_{T, 2 T}^{\theta}\right\|_{L^{1}\left(\nu_{2 T}\right) \rightarrow L^{2}\left(\nu_{T}\right)}=\left\|w_{\theta} K_{T, 2 T}^{\star} w_{-\theta}\right\|_{L^{2}\left(\nu_{T}\right) \rightarrow L^{\infty}\left(\nu_{2 T}\right)} .
$$

Setting $\left(K_{s, t}^{\star}\right)^{-\theta}=w_{\theta} K_{s, t}^{\star} w_{-\theta}$, we further have by duality that

$$
\begin{aligned}
\| K_{s, t}^{\star} & \|_{L^{1}\left(\nu_{s}\right) \rightarrow L^{\infty}\left(\nu_{t}\right)} \\
\left\|\left(K_{s, t}^{\star}\right)^{-\theta}\right\|_{L^{2}\left(\nu_{s}\right) \rightarrow L^{2}\left(\nu_{t}\right)} & =\left\|K_{s, t}\right\|_{L^{1}\left(\nu_{t}\right) \rightarrow L^{\infty}\left(\nu_{s}\right)} \leq \psi(t-s), \\
\theta, t & \|_{L^{2}\left(\nu_{t}\right) \rightarrow L^{2}\left(\nu_{s}\right)} \leq \exp ((t-s) \chi(\theta)),
\end{aligned}
$$


where the identity in (3.22) holds since $\left(K_{s, t}^{\star}\right)^{-\theta}$ is the adjoint of $K_{s, t}^{\theta}: L^{2}\left(\nu_{t}\right) \rightarrow L^{2}\left(\nu_{s}\right)$. Recall that the adjoint $K_{s, t}^{\star}$ of each Markov operator $K_{s, t}$ is a non-negative linear operator. Further, our assumption that $\left(\nu_{s} K_{s, t}\right) \leq \nu_{t}$ for strictly positive $\left\{\nu_{s}\right\}$ when $T \leq s \leq t \leq 2 T$, yields for $f_{t, y}:=\left(1 / \nu_{t}(y)\right) \delta_{y}$ any $y \in V$ and $[-1,1]$-valued $g \in \mathcal{C}_{0}(V)$,

$$
\left|\left(K_{s, t}^{\star} g\right)(y)\right|=\left|\left\langle f_{t, y}, K_{s, t}^{\star} g\right\rangle_{\nu_{t}}\right|=\left|\left\langle g, K_{s, t} f_{t, y}\right\rangle_{\nu_{s}}\right| \leq\left\langle K_{s, t} f_{t, y}\right\rangle_{\nu_{s}}=\frac{\left(\nu_{s} K_{s, t}\right)(y)}{\nu_{t}(y)} \leq 1 .
$$

Thus, $\left\|K_{s, t}^{\star}\right\|_{L^{\infty}\left(\nu_{s}\right) \rightarrow L^{\infty}\left(\nu_{t}\right)} \leq 1$, with (3.21) and (3.22) allowing us to apply Proposition 3.1 with $\varphi(\tau)=\psi(\tau)^{1 / 2}$ for the adjoint operators on time interval $[T, 2 T]$, to get that

$$
\left\|\left(K_{T, 2 T}^{\star}\right)^{-\theta}\right\|_{L^{2}\left(\nu_{T}\right) \rightarrow L^{\infty}\left(\nu_{2 T}\right)} \leq C_{2} \psi(T)^{\frac{1}{2}} \exp \left(C_{1} \chi(\theta) T\right) .
$$

With $C_{2}^{\prime}=C_{2}^{2}$, upon combining the latter bound with (3.19) and (3.20) we deduce that

$$
\left\|K_{0,2 T}^{\theta}\right\|_{L^{1}\left(\nu_{2 T}\right) \rightarrow L^{\infty}\left(\nu_{0}\right)} \leq C_{2}^{\prime} \psi(T) \exp \left(2 C_{1} \chi(\theta) T\right) .
$$

Since $\rho(y)-\rho(x)=d(y, x)$, specializing this operator bound to test function $f(y)=\delta_{y}$ yields

$$
K_{0,2 T}(x, y) e^{\theta d(x, y)} \leq C_{2}^{\prime} \psi(T) \exp \left(2 C_{1} \chi(\theta) T\right) \nu_{2 T}(y) \quad \forall y \in V .
$$

In view of (3.4), taking $\theta=d(x, y) /(\kappa T) \leq \delta_{\star}$ in (3.26), establishes the bound (3.18).

The next lemma, is part of the (discrete) integral maximum principle of [CGZ, Theorem 2.2, Proposition 2.3] and key to our proof of the Gaffney bound (3.3).

Lemma 3.4. Suppose $\pi$ is $\sigma$-finite measure and $K$ is a $\pi$-reversible, bounded range Markov transition on $V$. Then, for $f$ strictly positive and $u \in \mathcal{C}_{0}(V)$,

$$
2\langle f u(K u-u)\rangle_{\pi} \leq \frac{1}{4}\left\langle u^{2} f^{-1} K|\nabla f|^{2}\right\rangle_{\pi},
$$

and for $\alpha_{l}$-uniformly lazy $K$, also

$$
\left\langle f(K u)^{2}-f u^{2}\right\rangle_{\pi} \leq \frac{1}{4 \alpha_{l}}\left\langle u^{2} f^{-1} K|\nabla f|^{2}\right\rangle_{\pi} .
$$

Proof. For bounded range $K(x, y)$, any $u \in \mathcal{C}_{0}(V)$ and $f$, the function

$$
(K(\nabla u \nabla f))(x):=\sum_{y \in V} K(x, y)(u(y)-u(x))(f(y)-f(x))
$$

is in $\mathcal{C}_{0}(V)$. Following the algebra of [CGZ, Eq. (2.7)-(2.8)], if such $K(x, y)$ is $\pi$-reversible then

$$
2\langle f u(K u-u)\rangle_{\pi}=-\langle K(\nabla f u)(\nabla u)\rangle_{\pi}=-\left\langle f K|\nabla u|^{2}\right\rangle_{\pi}-\langle u K(\nabla u \nabla f)\rangle_{\pi} .
$$

Further, as in [CGZ, proof of Theorem 2.2], for strictly positive $\alpha f$,

$$
-\alpha\left\langle f K|\nabla u|^{2}\right\rangle_{\pi}-\langle u K(\nabla u \nabla f)\rangle_{\pi} \leq \frac{1}{4 \alpha}\left\langle u^{2} f^{-1} K|\nabla f|^{2}\right\rangle_{\pi}
$$

which for $\alpha=1$ yields (3.27) when combined with (3.30). Next recall as in [CGZ, Eq. (2.9)], that for $\alpha_{l}$-uniformly lazy Markov transition $K$ and any $u \in \mathcal{C}_{0}(V)$, by Cauchy-Schwarz

$$
(K u-u)^{2}(x) \leq\left(1-\alpha_{l}\right)\left(K|\nabla u|^{2}\right)(x), \quad \forall x \in V .
$$

Multiplying by $f(\cdot) \geq 0$ and integrating over the $\sigma$-finite measure $\pi$, results with

$$
\left\langle f(K u-u)^{2}\right\rangle_{\pi} \leq\left(1-\alpha_{l}\right)\left\langle f K|\nabla u|^{2}\right\rangle_{\pi} .
$$


For bounded range $K(\cdot, \cdot)$ all functions are in $\mathcal{C}_{0}(V)$, so combining (3.30), (3.32) and (3.31) we have

$$
\begin{aligned}
\left\langle f(K u)^{2}\right\rangle_{\pi}-\left\langle f u^{2}\right\rangle_{\pi} & =\left\langle f(K u-u)^{2}\right\rangle_{\pi}+2\langle f u(K u-u)\rangle_{\pi} \\
& \leq-\alpha_{l}\left\langle f K|\nabla u|^{2}\right\rangle_{\pi}-\langle u K(\nabla u \nabla f)\rangle_{\pi} \leq \frac{1}{4 \alpha_{l}}\left\langle u^{2} f^{-1} K|\nabla f|^{2}\right\rangle_{\pi},
\end{aligned}
$$

as stated in (3.28).

We proceed to establish the Gaffney bound (3.3) for non-decreasing $t \mapsto \pi_{t} \in \mathcal{M}_{+}(V)$ and bounded range $\pi_{t}$-reversible Markov operators $K_{t}$.

Lemma 3.5. [The Gaffney lemma] Suppose that Markov operators $K_{t}$ :

(a) have reversible measures $\pi_{t} \in \mathcal{M}_{+}(V)$ with $t \mapsto \pi_{t}(x)$ non-decreasing for any $x \in V$.

(b) have uniformly bounded range. That is, for some $r_{0}<\infty$

$$
\left\{y \in V: K_{t}(x, y)>0\right\} \subset \mathbb{B}\left(x, r_{0}\right), \quad \forall x \in V, t \in \mathbb{N} .
$$

(c) in case of discrete time, also $\inf _{t, x}\left\{K_{t}(x, x)\right\} \geq \alpha>0$.

Then, the $2 \rightarrow 2$ bound (3.3) holds for $K_{s, t}^{\theta}: L^{2}\left(\pi_{t}\right) \rightarrow L^{2}\left(\pi_{s}\right)$ provided

$$
L_{\rho}:=\sup _{x \neq y \in V}\left\{\frac{|\rho(x)-\rho(y)|}{d(x, y)}\right\}<\infty
$$

where $a_{t} \equiv 0$, for the dynamics (1.5) we have $\delta_{\star}=1, \alpha=1$ and $\chi(\cdot)=\zeta(\cdot)$ for

$$
\zeta(\theta):=\frac{1}{8 \alpha}\left(e^{2 r_{0} L_{\rho}|\theta|}-1\right)^{2},
$$

whereas for (1.3) set $\delta_{\star}=\infty$ and $\chi(\theta)=c_{1} \theta^{2}$ with $c_{1}:=\frac{1}{2} \sup _{\theta}\left\{\theta^{-2} \log (1+2 \zeta(\theta))\right\}$ finite.

Proof. For the $L^{2}\left(\pi_{t}\right)$-closure of non-negative $K_{s, t}^{\theta}$ it suffices to get (3.3) for $0 \leq g \in \mathcal{C}_{0}(V)$, namely

$$
e^{2 \chi(\theta) s}\left\|K_{s, t}^{\theta} g\right\|_{L^{2}\left(\pi_{s}\right)}^{2} \leq e^{2 \chi(\theta) t}\left\|K_{t, t}^{\theta} g\right\|_{L^{2}\left(\pi_{t}\right)}^{2}, \quad \forall s \in[0, t], \quad 0 \leq g \in \mathcal{C}_{0}(V) .
$$

For $f_{s}(x):=w_{-2 \theta}(x) e^{2 \chi(\theta) s}$ and $u \geq 0$ solving (1.23) on $[0, t] \times V$, with $u_{t}(\cdot)=u(t, \cdot) \in \mathcal{C}_{0}(V)$, let

$$
E_{s}(u):=\sum_{x \in V} f_{s}(x) u_{s}^{2}(x) \pi_{s}(x), \quad s \in[0, t] .
$$

In particular, (3.35) amounts to $E_{s}\left(u^{(\infty)}\right) \leq E_{t}\left(u^{(\infty)}\right)$ for $u_{s}^{(\infty)}=K_{s, t} u_{t}^{(\infty)}$ and $u_{t}^{(\infty)}=w_{\theta} g \in \mathcal{C}_{0}(V)$ (which for CSRW is absolutely continuous, see (1.5)). For large enough $R_{k} \uparrow \infty$ consider the solution $u^{(k)} \geq 0$ of (1.23) on $Q:=Q\left(0, t ; z, R_{k}\right)$ with $u_{t}^{(k)}=u_{t}^{(\infty)}$ and $u^{(k)} \equiv 0$ outside $Q$ (which correspond to the transition probabilities (1.3) or (1.5), killed at exiting $\mathbb{B}\left(z, R_{k}\right)$ ). By monotone convergence $E_{s}\left(u^{(k)}\right) \uparrow E_{s}\left(u^{(\infty)}\right)$ with equality at $s=t$ and $k$ large (c.f. [Fo, (3.10)] for such argument), and we thus proceed to show more generally that $s \mapsto E_{s}(u)$ is non-decreasing on $[0, t]$ for any solution $u$ of (1.23) on finite time-space cylinder $Q$, with zero boundary conditions (hence with $u_{s} \in \mathcal{C}_{0}(V)$ at any $\left.s \leq t\right)$. To this end, with $f_{s} \geq 0$ and non-decreasing $s \mapsto \pi_{s}(x)$ bounded on $Q$, clearly $f_{s} u_{s}^{2} \pi_{s} \leq f_{t} u_{t}^{2} \pi_{t}+\int_{s}^{t} \pi_{\xi} \partial_{-\xi}\left(f_{\xi} u_{\xi}^{2}\right) d \xi$ at each $(s, x) \in Q$. Thus, it suffices to show that (as a distribution in case of CSRW),

$$
\Delta E_{s}(u):=\left\langle\partial_{-s}\left(f_{s} u_{s}^{2}\right)\right\rangle_{\pi_{s}} \leq 0, \quad \text { for a.e. } s \in(0, t] .
$$

With $f_{s^{\prime}}$ strictly positive and $\rho$ Lipschitz, by the uniform bounded range assumption (3.33),

$$
\frac{1}{8 \alpha} f_{s^{\prime}}^{-1} K_{s}\left(\nabla f_{s^{\prime}}\right)^{2} \leq \zeta(\theta) f_{s^{\prime}}, \quad \forall s, s^{\prime} \geq 0
$$


For the dynamics of (1.3), since $u_{s^{\prime}}=K_{s} u_{s}$ and $f_{s}=e^{2 \chi(\theta)} f_{s^{\prime}}$ for $s^{\prime}=s-1$, we have that

$$
\Delta E_{s}(u)=\left\langle f_{s^{\prime}} u_{s^{\prime}}^{2}-f_{s} u_{s}^{2}\right\rangle_{\pi_{s}}=\left\langle f_{s^{\prime}}\left(K_{s} u_{s}\right)^{2}-f_{s^{\prime}} u_{s}^{2}\right\rangle_{\pi_{s}}-\left\langle h_{s} u_{s}^{2}\right\rangle_{\pi_{s}}
$$

where $h_{s}:=\left(e^{2 \chi(\theta)}-1\right) f_{s^{\prime}}$. Similarly, for the dynamics of (1.5), since $\partial_{-s} f_{s}=-2 \chi(\theta) f_{s}$ and a.e. $\partial_{-s} u_{s}=\left(K_{s}-I\right) u_{s}$ (unless $u_{s}=0$ by our zero boundary condition), we find that a.e.

$$
\Delta E_{s}(u)=\left\langle 2 u_{s} f_{s} \partial_{-s} u_{s}+u_{s}^{2} \partial_{-s} f_{s}\right\rangle_{\pi_{s}}=2\left\langle u_{s} f_{s}\left(K_{s}-I\right) u_{s}\right\rangle_{\pi_{s}}-\left\langle h_{s} u_{s}^{2}\right\rangle_{\pi_{s}},
$$

now with $h_{s}:=2 \chi(\theta) f_{s}$. In view of (3.38), taking $\alpha=1, \chi_{c}(\cdot)=\zeta(\cdot)$ and $s^{\prime}=s$ in the continuous time setting, while $\chi_{d}(\theta)=c_{1} \theta^{2} \geq \frac{1}{2} \log (1+2 \zeta(\theta))$ in discrete time, yields that in both cases

$$
\frac{1}{4 \alpha}\left\langle u_{s}^{2} f_{s^{\prime}}^{-1} K_{s}\left|\nabla f_{s^{\prime}}\right|^{2}\right\rangle_{\pi_{s}} \leq\left\langle u_{s}^{2} h_{s}\right\rangle_{\pi_{s}} .
$$

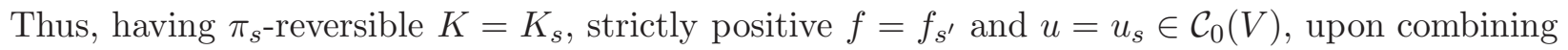
(3.27) and (3.40), or (3.28) and (3.39), we get (3.37) for both the continuous and discrete time dynamics. To complete the proof of the lemma, just confirm that (3.4) holds for $\chi_{c}(\cdot)=\zeta(\cdot)$, $\delta_{\star}=1$ and some $c_{1}$ finite.

Proof of Theorem 1.8(a): In case of lazy DTRW it suffices to consider $d(x, y) \leq t-s$ where the bound of (1.27) is merely the conclusion of Proposition 3.3 for $\psi(k)=C^{\prime} / v(\sqrt{2 k}), \delta_{\star}=\infty$, the dynamic (1.3) for $\left\{\left(K_{r+s}, \pi_{r+s}\right): r \in(0, t-s]\right\}$ and $\nu_{r}=\pi_{r}$. Indeed, the required $1 \rightarrow \infty$ bound (3.17) is provided by Lemma 2.8, whereas the $2 \rightarrow 2$ Gaffney bound of (3.3) is proved for $a_{t} \equiv 0$ and $\rho(\cdot)=d(\cdot, x)$, in Lemma 3.5. The same applies for the CSRW, except that now $\delta_{\star}=1$ in the $2 \rightarrow 2$ Gaffney bound, hence also in (3.18). Nevertheless, in this case (3.26) holds with $\chi(\theta) \leq \frac{1}{4} \exp (4|\theta|)$, so considering $\theta=\frac{1}{4} \log \left(d(x, y) /\left(2 C_{1} T\right)\right)$ for $T=(t-s) / 2$ yields the stated bound (1.27).

Further use of the integral maximum principle as in [CGZ, Prop. 2.5] yields the following lemma that we shall use in the sequel to strengthen the preceding GHKU.

Lemma 3.6. Let $I(r)=r^{2}$ for the dynamics (1.3) and $I(\cdot)$ as in Theorem 1.8 (a) for the dynamics (1.5). Then, in the setting of Lemma 3.5, for

$$
f_{s}(x):=\exp (-\eta(s+1) I(\rho(x) /(s+1))), \quad x \in V, s \in \mathbb{Z}_{+},
$$

the function $s \mapsto E_{s}(u)$ of $(3.36)$ is non-decreasing provided $\inf _{x} \rho(x) \geq 1, u_{t} \in \mathcal{C}_{0}(V)$, and $\eta \in\left[0, c_{2}^{-1}\right]$ for some $c_{2}\left(L_{\rho} r_{0}, \alpha\right)$ finite.

Proof. Following the proof of Lemma 3.5, consider first the discrete dynamic (1.3). Then, by (3.28) and (3.39) it suffices to find $c_{2}<\infty$ such that for $c_{2} \eta \in[0,1]$ and $s^{\prime}=s-1 \geq 0$

$$
\frac{1}{4 \alpha} f_{s^{\prime}}^{-1}\left(K_{s}\left|\nabla f_{s^{\prime}}\right|^{2}\right) \leq h_{s}
$$

where for the strictly positive $f_{s}$ of (3.41), at $x \in V$,

$$
h_{s}(x):=f_{s}(x)-f_{s^{\prime}}(x)=f_{s^{\prime}}(x)\left(e^{\frac{\eta \rho(x)^{2}}{\left(s^{\prime}+1\right)(s+1)}}-1\right) .
$$

Next, if $d(x, y) \leq r_{0}$ then $|\rho(x)-\rho(y)| \leq L_{\rho} r_{0}$ and as $\rho(x) \geq 1$ also $\left|\rho(x)^{2}-\rho(y)^{2}\right| \leq c_{3} \rho(x)$ for $c_{3}:=L_{\rho} r_{0}\left(2+L_{\rho} r_{0}\right)$ finite. In this case, the inequality $\left|e^{w}-1\right| \leq e^{|w|}-1$ yields

$$
\left|f_{s^{\prime}}(y)-f_{s^{\prime}}(x)\right| \leq f_{s^{\prime}}(x)\left(e^{\frac{|\eta|\left|\rho(x)^{2}-\rho(y)^{2}\right|}{s^{\prime}+1}}-1\right) \leq f_{s^{\prime}}(x)\left(e^{\frac{c_{3}|\eta| \rho(x)}{s^{\prime}+1}}-1\right) .
$$


Next, recall that for any $\alpha>0$ there exists $b(\alpha)$ finite, such that $(4 \alpha)^{-1}\left(e^{w}-1\right)^{2} \leq e^{b(\alpha) w^{2}}-1$ for any $w \geq 0$. Thus, by assumption (3.33), the LHS of (3.42) is bounded above by

$$
f_{s^{\prime}}(x)\left(e^{\frac{b(\alpha) c_{3}^{2} \eta^{2} \rho(x)^{2}}{\left(s^{\prime}+1\right)^{2}}}-1\right) \leq h_{s}(x)
$$

provided non-negative $\eta \leq\left(2 b(\alpha) c_{3}^{2}\right)^{-1}$ is chosen in (3.41). Turning to the dynamic (1.5), by (3.27) and (3.40) it similarly suffices to show that

$$
\sup _{y \in \mathbb{B}\left(x, r_{0}\right)}\left|f_{s}(x)-f_{s}(y)\right|^{2} \leq 2 \partial_{s} f_{s}^{2}(x),
$$

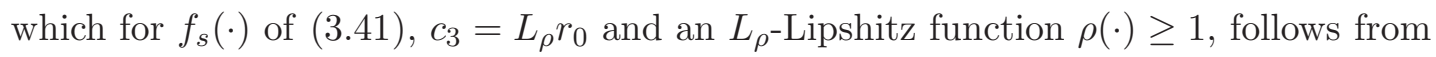

$$
\sup _{\delta \in(0, r]} \sup _{\left|r^{\prime}-r\right| \leq c_{3} \delta}\left\{e^{\eta\left|I\left(r^{\prime}\right)-I(r)\right| / \delta}-1\right\}^{2} \leq 4 \eta\left[r I^{\prime}(r)-I(r)\right]
$$

(take $\delta^{-1}=(s+1), r=\rho(x) \delta$ and $\left.r^{\prime}=\rho(y) \delta\right)$. Further, with $r I^{\prime}(r)-I(r)=r(r \wedge 1)$ and $I^{\prime}(r)=2(r \wedge 1)+(\log r)_{+}$non-decreasing on $\mathbb{R}_{+}$, it suffices in turn to verify that

$$
g(r):=\exp \left(\eta c_{3} I^{\prime}\left(\left(1+c_{3}\right) r\right)\right)-1-2 \sqrt{\eta r(r \wedge 1)} \leq 0, \quad \forall r>0 .
$$

To this end, note that $g(0)=0$ and it is not hard to check that $g^{\prime}(r) \leq 0$ whenever $2 \eta c_{3} \leq 1$ and $\sqrt{\eta} c_{3}\left(1+c_{3}\right) e \leq 1$. That is, for any non-negative $\eta \leq 1 / c_{2}\left(c_{3}\right)$, as claimed.

\section{Parabolic Harnack Inequality}

We adapt here Grigor'yan's approach $[\mathrm{Gr}$ to proving PHI to the case of continuous time heat equation (1.23) on graphs associated with the CSRW, when $t \mapsto \pi_{t}(x)$ are non-decreasing and uniformly bounded. Building on weighted Poincaré and $L^{2}$-mean-value inequalities, the crucial element of the proof is a first growth lemma (here Lemma 4.4). Combining such first growth lemma with the uniform volume doubling condition, one then derives the second growth lemma (here Lemma 4.77), which yields the Harnack inequality by a quite intricate, but by now classical, argument. We thus proceed with the weighted Poincaré inequality of [Del, Prop. 2.2].

Proposition 4.1. [Weighted Poincaré inequality] Suppose $\pi$ has VD property with constant $\mathrm{C}_{\mathrm{D}}$ and the Poincare inequality with constant $\mathrm{C}_{\mathrm{P}}$ holds for uniformly elliptic, $\pi$-reversible, Markov transition $K(\cdot, \cdot)$ on $E$. Then, there exist $\mathrm{C}_{\mathrm{P}}^{\prime}\left(\mathrm{C}_{\mathrm{P}}, \mathrm{C}_{\mathrm{D}}, \alpha_{e}\right)$ finite, such that for $\mathbb{B}:=\mathbb{B}(z, 2 r)$ and $\eta(\cdot):=\left\{[1-d(\cdot, z) /(2 r)]_{+}\right\}^{2}$,

$$
\mathrm{C}_{\mathrm{P}}^{\prime} r^{2}\left\langle\left(\eta_{\wedge} K\right)|\nabla f|^{2}\right\rangle_{\pi} \geq \frac{\pi\left(\mathbb{H}_{f}\right)}{\pi(\mathbb{B})}\left\langle\eta f^{2}\right\rangle_{\pi} \quad \forall r>0, z \in V, f: V \rightarrow \mathbb{R}_{+}
$$

where $\mathbb{H}_{f}:=\mathbb{B}(z, r) \cap f^{-1}(\{0\}), \eta_{\wedge}(x, y):=\eta(x) \wedge \eta(y)$ and $\left(\eta_{\wedge} K\right)|\nabla f|^{2}$ is as in (3.29).

Proof. From [Del, Prop. 2.2] we have the weighted Poincaré inequality

$$
\frac{\mathrm{C}_{\mathrm{P}}^{\prime}}{4} r^{2}\left\langle\left(\eta_{\wedge} K\right)|\nabla f|^{2}\right\rangle_{\pi} \geq\left\langle\eta f^{2}\right\rangle_{\pi}-\frac{\langle\eta f\rangle_{\pi}^{2}}{\langle\eta\rangle_{\pi}} \geq \frac{\left\langle\eta \mathbf{1}_{\{f=0\}}\right\rangle_{\pi}}{\langle\eta\rangle_{\pi}}\left\langle\eta f^{2}\right\rangle_{\pi},
$$

where the right-inequality is merely Cauchy-Schwarz for $f \mathbf{1}_{f>0}$. Since the $[0,1]$-valued $\eta(\cdot)$ is supported on $\mathbb{B}$ and exceeds $1 / 4$ throughout $\mathbb{B}(z, r)$, we arrive at (4.1). 
The next ingredient is $L^{2}$-mean value inequality (denoted $\mathrm{ML}^{2}$ ), analogous to the one in $\mathrm{CG}$, Sect. 4.1] for uniformly lazy DTRW on time-invariant graph. To this end, recall first that a $\pi$ reversible Markov transition $K(x, y)$ satisfies a relative Faber-Krahn (FK) inequality if there exist positive $a, \nu$ such that

$$
\lambda_{K}(\Omega) \geq \frac{a}{r^{2}}\left(\frac{\pi(\mathbb{B})}{\pi(\Omega)}\right)^{\nu}, \quad \forall r>0, z \in V, \Omega \subseteq \mathbb{B},|\Omega| \geq 1,
$$

where $\lambda_{K}(\Omega)$ of (2.26) is the smallest eigenvalue of $I-K$ with Dirichlet boundary condition in $\Omega$. By [CG, Proposition 2.3], the FK inequality (4.2) follows from the VD property and Poincaré inequality, with constants $a, \nu$ that depend only on $C_{D}$ and $C_{P}$. Proceeding to adapt the relevant part of [CG, Sect. 4] to our continuous time-varying setting, for Markov kernels $\left\{K_{t}\right\}$ of uniformly bounded range (as in (3.33) ), we denote by $\boldsymbol{\pi}(\cdot)$ the $\sigma$-finite measure on $[0, \infty) \times V$ such that

$$
\boldsymbol{\pi}(S)=\int_{0}^{\infty}\left[\sum_{x \in V} \mathbf{1}_{\{(t, x) \in S\}} \pi_{t}(x)\right] d t
$$

and call $u: Q \mapsto \mathbb{R}_{+}$a super-solution (of the heat equation) on $Q=Q\left(t_{1}, t_{2} ; z, R\right)$ of (1.22) if

$$
\partial_{-s} u(s, x) \geq \sum_{y} K_{s}(x, y) u(s, y)-u(s, x), \quad \forall(s, x) \in Q
$$

for some non-negative boundary values outside $Q$ (restricting to $s \in \mathbb{N}$ in discrete time, while for CSRW the inequality is between distributions and holds a.e.). Similarly, $u \geq 0$ is called a sub-solution on $Q$ when the reversed inequality (4.3) holds (see [Del, Sec. 2.2]).

Remark 4.2. If $u \geq 0$ is a solution of (1.23) on $Q$, it must satisfy there (1.5) for CSRW stopped upon exiting $\mathbb{B}(z, R)$. For any $\Phi(\cdot)$ convex, $v=\Phi(u)$ is then absolutely continuous on $Q$, and by Jensen's inequality has LHS $\leq$ RHS in (1.5) (throughout $Q$ ). Taking $s \uparrow t$ we deduce that $v$ is a sub-solution on $Q$. Likewise, $v=\Phi(u)$ is a super-solution on $Q$ whenever $\Phi(\cdot)$ is concave.

Proposition 4.3. $\left[L^{2}\right.$-mean value inequality $]$

Suppose $t \mapsto \pi_{t}(x)$ is non-decreasing with $\mathrm{C}_{0}:=\sup _{t, x}\left\{\frac{\pi_{t}(x)}{\pi_{0}(x)}\right\}$ finite and the $\pi_{t}$-reversible, Markov operators $K_{t}$ satisfy (3.33) and the relative $\mathrm{FK}$ inequality with same positive $a, \nu$. Then, for $\vartheta(t):=$ $\max \left\{t, t^{-1 / \nu}\right\}$, some $C=C\left(a, \nu, \mathrm{C}_{0}\right)<\infty$ any $T \geq 2 t \geq 4, R>r_{0}, z \in V$ and sub-solution $u(\cdot, \cdot)$ on $Q:=Q(T-2 t, T ; z, R)$ of (4.3),

$$
\mathrm{ML}^{2}: \quad u^{2}(T-t, z) \leq \frac{C \vartheta\left(t / R^{2}\right)}{\pi(Q)} \int_{Q} u^{2} d \boldsymbol{\pi} .
$$

Proof. We follow closely the argument in CG, Sect. 4], starting with the analogue of [CG, Corollary 4.7]. To this end, for any functions $u, g$ on $V$,

$$
|\nabla(g u)|^{2}-\left(\nabla g^{2} u\right)(\nabla u)=u(x) u(y)|\nabla g|^{2} \leq \frac{1}{2} u^{2}(x)|\nabla g|^{2}+\frac{1}{2} u^{2}(y)|\nabla g|^{2} .
$$

Hence, for any $\pi$-reversible operator $K(x, y)$ on $V$ and $g \in \mathcal{C}_{0}(V)$,

$$
2 \mathcal{E}_{K, \pi}(g u, g u)+2\left\langle g^{2} u(K u-u)\right\rangle_{\pi} \leq\left\langle u^{2} K|\nabla g|^{2}\right\rangle_{\pi}
$$

(recall (2.18) and the LHS of (3.30) $)$. Fix any $\eta(s, x)$ supported on finite time-space region $[T-$ $2 t, T] \times \Omega$ with $\eta(T, \cdot) \equiv 0$ and $\left\|(\nabla \eta)^{2}\right\|_{\infty}+\left\|\partial_{s} \eta^{2}\right\|_{\infty} \leq M$. Since $s \mapsto \pi_{s}(x)$ are non-decreasing, from (4.5) for $g=\eta_{s}=\eta(s, \cdot)$ differentiable in $s$, any sub-solution $u_{s}=u(s, \cdot)$ and the $\pi_{s}$-reversible $K_{s}$, we get that at a.e. $s \in[T-2 t, T]$,

$$
2 \mathcal{E}_{K_{s}, \pi_{s}}\left(\eta_{s} u_{s}, \eta_{s} u_{s}\right)+\partial_{-s}\left\langle\eta_{s}^{2} u_{s}^{2}\right\rangle_{\pi_{s}} \leq\left\langle u_{s}^{2} K_{s}\left|\nabla \eta_{s}\right|^{2}\right\rangle_{\pi_{s}}+\left\langle u_{s}^{2} \partial_{-s} \eta_{s}^{2}\right\rangle_{\pi_{s}}
$$


Integrating both sides over $[T-\tau, T]$ yields the analogue of [CG, Eq. (4.15)]. That is, for $\widetilde{\Omega}=\{z \in$ $\left.V: d(z, \Omega) \leq r_{0}\right\}$ and any $\tau \leq 2 t$,

$$
\|\eta u\|_{L^{2}\left(\pi_{T-\tau}\right)}^{2}+2 \int_{T-\tau}^{T} \mathcal{E}_{K_{s}, \pi_{s}}(\eta u, \eta u) d s \leq 2 M \int_{[T-\tau, T] \times \widetilde{\Omega}} u^{2} d \boldsymbol{\pi} .
$$

We proceed to adapt the proof from [CG, Sect. $4.4 \& 4.5]$ of the $\mathrm{ML}^{2}$. Indeed, by the assumed monotonicity of $s \mapsto \pi_{s}$ and uniformity of $a, \nu$, here the relative Faber-Krahn inequality (4.2) yields that for any $s \geq 0, z \in V, r>0$ and non-empty $\Omega \subseteq \mathbb{B}(z, r)$,

$$
\lambda_{K_{s}}(\Omega) \geq \Lambda\left(\pi_{s}(\Omega)\right), \quad \Lambda(\xi):=\frac{a}{r^{2}} \pi_{0}(\mathbb{B}(z, r))^{\nu} \xi^{-\nu} .
$$

With (4.6) and (4.7) taking the roles of [CG, Eq. (4.15)] and [CG, Eq (4.19)], respectively, the proof of [CG, Eq. (4.20)] applies verbatim, upon changing on [CG, page 681] to $I:=\int_{\Psi} u^{2} d \boldsymbol{\pi}$, $I^{\prime}:=\int_{\Psi^{\prime}}(u-\theta)_{+}^{2} d \boldsymbol{\pi}$, for a solution $u(\cdot, \cdot)$, constant $\theta>0$ and invoking hereafter the time inversion $s \mapsto(T-s)$ on $\Psi^{\prime} \subseteq \Psi$ and all other time-space cylinders from [CG]. We proceed as in [CG, pages 685-687] to compare via [CG, Eq. (4.20)] the values of $I_{n-1}$ and $I_{n}:=\int_{\Psi_{n}}\left(u-\theta_{n}\right)_{+}^{2} d \boldsymbol{\pi}$, for $\theta_{n}=\theta\left(2-2^{-n}\right)$ and decreasing cylinders $\Psi_{n}:=Q\left(T-2 t+n, T-t+t_{n} ; z, R_{n}\right)$, with $t_{n}=t 2^{-n}$ and $R_{n}=\left\lceil R_{n-1} / 2\right\rceil$, starting at $\Psi_{0}=Q$. Iterating to $N=\max \left\{n: R_{n} \geq r_{0}+1, t_{n} \geq 2\right\}$, we arrive at [CG, Eq. (4.36)] where $\beta:=\Lambda(1) \leq \pi_{0}(z)^{\nu}$ by (4.2) for $\Omega=\{z\} \quad$ as $\left.\lambda_{K_{0}}(\{z\}) \leq 1\right)$. Setting $M=2, \hat{\tau}=2$ and $\hat{T}-\hat{\tau}=T-t$, consider (4.6) for the sub-solution $\widehat{u}:=\left(u-2 \theta_{N}\right)_{+}$ (recall Remark 4.2), and [0,1]-valued $\hat{\eta}$ supported on $[\hat{T}-2 \hat{\tau}, \hat{T}) \times \Omega$, such that $\widehat{\eta}(T-t, z)=1$ and $\left\|(\nabla \hat{\eta})^{2}\right\|_{\infty}+\left\|\partial_{s} \hat{\eta}^{2}\right\|_{\infty} \leq M$. Since $\widetilde{Q}=[\hat{T}-\hat{\tau}, \hat{T}] \times \widetilde{\Omega} \subset \Psi_{N}$ and $\theta_{N} \leq 2 \theta$, we have that

$$
(u(T-t, z)-2 \theta)_{+}^{2} \pi_{0}(z) \leq 2 M \int_{\widetilde{Q}} \hat{u}^{2} d \boldsymbol{\pi} \leq 2 M I_{N} .
$$

Continuing as in [CG], we cancel the common power of $m(z)=\pi_{0}(z)$ from both sides of [CG, Eq. (4.38)], en-route to [CG, Eq. (4.39)] and thereby to $\mathrm{ML}^{2}$ by taking $\theta=\frac{1}{3} u(T-t, z)$.

Having the key ingredients of Prop. 4.1 and Prop. 4.3, we now establish the first growth lemma.

Lemma 4.4. [First growth lemma] Suppose $\left\{\boldsymbol{G}_{t}\right\}$ are as in Theorem 1.6.

For any $\delta>0$ there exists $\varepsilon=\varepsilon\left(\delta, C_{P}, C_{D}, \alpha_{e}, C_{0}\right)>0$ such that for all $T \geq 6 R^{2}, z \in V$ and any positive solution $u(\cdot, \cdot)$ of (1.23) on $Q:=Q\left(T-4 R^{2}, T ; z, 2 R\right)$,

$$
\frac{\boldsymbol{\pi}\left(Q\left(T-R^{2}, T ; z, R\right) \cap u^{-1}([1, \infty))\right)}{\boldsymbol{\pi}\left(Q\left(T-R^{2}, T ; z, R\right)\right)} \geq \delta \quad \Longrightarrow \quad \inf _{Q\left(T-3 R^{2}, T-2 R^{2} ; z, R\right)} u \geq \varepsilon .
$$

Proof. Fixing $z \in V$ and $T \geq 4 R^{2}$ set $\mathbb{B}:=\mathbb{B}(z, 2 R)$ and $\eta(\cdot):=\left\{[1-d(\cdot, z) /(2 R)]_{+}\right\}^{2}$ as in Prop. 4.1. Recall Remark 1.5 that any solution $u>0$ of (1.23) on $Q$ can be replaced by bounded away from zero solutions $u_{b}=(1-b) u+b$, without altering the LHS of (4.9). Hence, by Remark 4.2, WLOG we have the associated super-solution $\widetilde{u}:=1 \wedge u \geq b$ for some $b>0$, and uniformly bounded sub-solution $v:=-\log \widetilde{u}$. Consider the functions $F(s):=\left\langle\eta v_{s}\right\rangle_{\pi_{s}}$ on $\left[T-4 R^{2}, T\right], \widetilde{u}_{s}:=\widetilde{u}(s, \cdot)$ and $v_{s}:=v(s, \cdot)$ on $V$ and the subset $\mathbb{H}_{v_{s}}=\mathbb{B}(z, R) \cap u(s, \cdot)^{-1}([1, \infty))$ of $\mathbb{B}$. Having $s \mapsto \pi_{s}(\cdot)$ non-decreasing and $\widetilde{u} \geq b$ a super-solution, it follows from (4.3) and the LHS of (3.30) that as distributions, for a.e. $s$,

$$
\partial_{s} F(s) \geq\left\langle\frac{\eta}{\widetilde{u}_{s}} \partial_{-s} \widetilde{u}_{s}\right\rangle_{\pi_{s}} \geq\left\langle\frac{\eta}{\widetilde{u}_{s}} K_{s} \nabla \widetilde{u}_{s}\right\rangle_{\pi_{s}}=-\frac{1}{2}\left\langle K_{s}\left(\nabla\left(\frac{\eta}{\widetilde{u}_{s}}\right) \nabla \widetilde{u}_{s}\right)\right\rangle_{\pi_{s}}
$$


Setting $\psi(c)=\frac{1}{2 c}$ for $c>0$ and $\psi(0)=1$, recall that for any $a, b>0$ and $c, d \geq 0$,

$$
-\left(\frac{d}{b}-\frac{c}{a}\right)(b-a) \geq \frac{1}{2}(c \wedge d)(\log b-\log a)^{2}-|d-c| \psi\left(\frac{c \wedge d}{|d-c|}\right)
$$

(see [SZh, Inequality (1.23)]). For $(x, y) \in E$ and $k \in \mathbb{Z}_{+}$, if $\eta_{\wedge}=k^{2}(2 R)^{-2}$ then necessarily $|\nabla \eta| \leq(2 k+1)(2 R)^{-2}$, so $|\nabla \eta| \psi\left(\eta_{\wedge} /|\nabla \eta|\right) \leq \frac{9}{8} R^{-2}$ (or zero, whenever $\left.d(z, x) \vee d(z, y)>2 R\right)$. Hence, upon summing over $\pi_{s} K_{s}$ we get as in [Ba, proof of (5.7)], that

$$
-\left\langle K_{s}\left(\nabla\left(\frac{\eta}{\widetilde{u}_{s}}\right) \nabla \widetilde{u}_{s}\right)\right\rangle_{\pi_{s}} \geq \frac{1}{2}\left\langle\left(\eta_{\wedge} K_{s}\right)\left|\nabla v_{s}\right|^{2}\right\rangle_{\pi_{s}}-\frac{9}{4} R^{-2} \pi_{s}(\mathbb{B}) .
$$

Next, by Prop. 4.1 for $v_{s} \geq 0$ and $r=R$, followed by Cauchy-Schwartz,

$$
\mathrm{C}_{\mathrm{P}}^{\prime} R^{2}\left\langle\left(\eta_{\wedge} K_{s}\right)\left|\nabla v_{s}\right|^{2}\right\rangle_{\pi_{s}} \geq \frac{\pi_{s}\left(\mathbb{H}_{v_{s}}\right)}{\pi_{s}(\mathbb{B})}\left\langle\eta v_{s}^{2}\right\rangle_{\pi_{s}} \geq \frac{\pi_{s}\left(\mathbb{H}_{v_{s}}\right)}{\pi_{s}(\mathbb{B})^{2}} F(s)^{2} .
$$

Plugging this into (4.10)-(4.11) yields

$$
\partial_{s} F(s) \geq L(s) F(s)^{2}-D(s), \quad L(s):=\frac{R^{-2} \pi_{s}\left(\mathbb{H}_{v_{s}}\right)}{\mathrm{C}_{\mathrm{P}}^{\prime}\left(2 \mathrm{C}_{0}\right)^{2} \pi_{0}(\mathbb{B})^{2}}, \quad D(s):=\frac{9}{8} R^{-2} \pi_{s}(\mathbb{B}) .
$$

Following [Gr, pg. 67], let $J(t)=F(t)-\int_{t}^{T} D(s) d s$ and $t_{\star}:=\sup \{t \leq T: J(t) \leq 0\}$. With $F$, $L, D$ non-negative and $J(T) \geq 0$, we have on $\left[t_{\star}, T\right]$ that $\partial_{s} J \geq L F^{2} \geq L J^{2}$ and consequently $J(t) \leq\left(\int_{t}^{T} L(s) d s\right)^{-1}$. Further $t \mapsto J(t)$ is non-decreasing and $J\left(t_{\star}\right)=0$, so this bound extends to all $t$. Thus, on $\left[T-4 R^{2}, T-R^{2}\right]$,

$$
F(t) \leq\left(\int_{T-R^{2}}^{T} L(s) d s\right)^{-1}+\int_{t}^{T} D(s) d s .
$$

From the LHS of (4.9) and definition of $\mathbb{H}_{v_{s}}$ we have $R^{-2} \int_{T-R^{2}}^{T} \pi_{s}\left(\mathbb{H}_{v_{s}}\right) d s \geq \delta \mathrm{C}_{\mathrm{D}}^{-1} \pi_{0}(\mathbb{B})$, hence the first term on the RHS of (4.12) is at most $\delta^{-1} C_{D} C_{P}^{\prime}\left(2 C_{0}\right)^{2} \pi_{0}(\mathbb{B})$. The other term is at most $7 \mathrm{C}_{0} \pi_{0}(\mathbb{B})$, so for some $C_{1}\left(C_{\mathrm{P}}^{\prime}, \mathrm{C}_{\mathrm{D}}, \mathrm{C}_{0}\right)$ finite,

$$
F(t) \leq C_{1} \pi_{0}(\mathbb{B}) \delta^{-1}, \quad \forall t \in\left[T-4 R^{2}, T-R^{2}\right]
$$

For $t \in\left[T-3 R^{2}, T-2 R^{2}\right]$, integrating (4.10) on $I_{t}:=\left[t-R^{2}, t+R^{2}\right]$, yields by (4.11) and (4.13) that

$$
\int_{I_{t}}\left\langle\left(\eta_{\wedge} K_{s}\right)\left|\nabla v_{s}\right|^{2}\right\rangle_{\pi_{s}} d s \leq 4 F\left(t+R^{2}\right)+\frac{9}{2} R^{-2} \pi\left(I_{t} \times \mathbb{B}\right) \leq C_{2} \pi_{0}(\mathbb{B}) \delta^{-1},
$$

where $C_{2}=4 C_{1}+9 C_{0}$. Since $\zeta:=\mathbf{1}_{\mathbb{B}^{\prime}} \leq 16 \eta$ for $\mathbb{B}^{\prime}:=\mathbb{B}(z, 3 R / 2)$, it follows that

$$
\bar{v}_{s}:=\frac{\left\langle v_{s} \zeta\right\rangle_{\pi_{s}}}{\langle\zeta\rangle_{\pi_{s}}} \leq \frac{16 F(s)}{\pi_{0}\left(\mathbb{B}^{\prime}\right)}, \quad \mathcal{E}_{K_{s}, \pi_{s}}\left(v_{s} \zeta, v_{s} \zeta\right) \leq 8\left\langle\left(\eta_{\wedge} K_{s}\right)\left|\nabla v_{s}\right|^{2}\right\rangle_{\pi_{s}}
$$

Recall that under uniform ellipticity, the VD property and (weak) Poincaré inequality (PI) of (1.10), implies the strong-PI where $\mathbb{B}\left(x_{0}, r\right)$ replaces $\mathbb{B}\left(x_{0}, 2 r\right)$ on the RHS of (1.10) (see [Ba2, Cor. A.51] or $\left[\right.$ Kum, Prop. 3.3.2]). From the strong-PI on $\mathbb{B}^{\prime}$ and the preceding bounds,

$$
\left\langle v_{s}^{2} \zeta\right\rangle_{\pi_{s}}=\bar{v}_{s}^{2}\langle\zeta\rangle_{\pi_{s}}+\left\langle\left(v_{s}-\bar{v}_{s}\right)^{2} \zeta\right\rangle_{\pi_{s}} \leq \frac{16^{2} F(s)^{2}}{\pi_{0}\left(\mathbb{B}^{\prime}\right)}+16 \mathrm{C}_{\mathrm{P}} R^{2}\left\langle\left(\eta_{\wedge} K_{s}\right)\left|\nabla v_{s}\right|^{2}\right\rangle_{\pi_{s}}
$$

Combining (4.13)-(4.15), we get for some $C_{3}\left(C_{1}, C_{2}, C_{\mathrm{D}}, \mathrm{C}_{\mathrm{P}}\right)$ finite and all $t \in\left[T-3 R^{2}, T-2 R^{2}\right]$,

$$
\int_{I_{t} \times \mathbb{B}^{\prime}} v^{2} d \boldsymbol{\pi} \leq C_{3} R^{2} \pi_{0}(\mathbb{B}) \delta^{-2}
$$


Applying the $L^{2}$-mean value of Prop. 4.3 to the sub-solution $v$ on $\left.I_{t} \times \mathbb{B}(x, R / 2)\right)$ together with the vD property of $\pi_{0}$, we get for $C_{i}=C_{i}\left(\mathrm{C}_{\mathrm{P}}, \mathrm{C}_{\mathrm{D}}, \alpha_{e}, \mathrm{C}_{0}\right)$ finite and all $(t, x) \in Q\left(T-3 R^{2}, T-2 R^{2} ; z, R\right)$,

$$
v^{2}(t, x) \leq \frac{C_{4} R^{-2}}{\pi_{0}(\mathbb{B}(x, R / 2))} \int_{I_{t} \times \mathbb{B}(x, R / 2)} v^{2} d \boldsymbol{\pi} \leq \frac{C_{5} R^{-2}}{\pi_{0}(\mathbb{B})} \int_{I_{t} \times \mathbb{B}^{\prime}} v^{2} d \boldsymbol{\pi} \leq C_{6}^{2} \delta^{-2},
$$

using (4.16) in the last step. That is, (4.9) holds with $\varepsilon=\exp \left(-C_{6} / \delta\right)>0$.

Adapting the derivation of [Gr, Lemma 4.3] (out of [Gr, Lemma 4.1]), yields the following consequence of Lemma 4.4 .

Lemma 4.5. For $\left\{\boldsymbol{G}_{t}\right\}$ as in Theorem 1.6 there exist finite $\eta=\eta\left(\mathrm{C}_{\mathrm{P}}, \mathrm{C}_{\mathrm{D}}, \alpha_{e}, \mathrm{C}_{0}\right)$ and $R_{0}=$ $R_{0}\left(\mathrm{C}_{\mathrm{P}}, \mathrm{C}_{\mathrm{D}}, \alpha_{e}, \mathrm{C}_{0}\right)$ such that for any $R \geq R_{0}$ and all $z, T, u(\cdot, \cdot)$ as in Lemma 4.4 ,

$$
\frac{\boldsymbol{\pi}\left(Q\left(T-R^{2}, T ; z, R\right) \cap u^{-1}([1, \infty))\right)}{\boldsymbol{\pi}\left(Q\left(T-R^{2}, T ; z, R\right)\right)} \leq \eta \& u\left(T-R^{2}, z\right) \geq 2 \quad \Longrightarrow \quad \sup _{Q\left(T-2 R^{2}, T ; z, R\right)} u \geq 4 .
$$

Remark 4.6. An alternative and quicker approach by Fabes-Stroock utilizes the weighted Poincaré inequality in a different way (e.g. [FS, $[\mathrm{Ba},[\mathrm{BK}]$ ). It relies on having a-priori that

$$
K_{t-s, t}(x, x) \leq \frac{C}{\pi_{0}(\mathbb{B}(x, \sqrt{s}))} \quad \& \quad \inf _{s, t, y} \sum_{x \in \mathbb{B}(y, C \sqrt{s})} K_{t-s, t}(x, y)>0
$$

(which take the role of (4.9) in proving the first growth lemma). However, lacking a uniform in $y$ lower bound on $\sum_{x \in \mathbb{B}(y, C \sqrt{s})} K_{t-s}(x, y)$, prevents using this approach in our time-varying setting.

Under uniform volume doubling condition, the first growth lemma implies second growth lemma, following the same proof as [Gr, Lemma 4.2] verbatim.

Lemma 4.7. [Second growth lemma] For $\left\{\boldsymbol{G}_{t}\right\}$ of Theorem [1.6, some $\theta=\theta\left(\mathrm{C}_{\mathrm{P}}, \mathrm{C}_{\mathrm{D}}, \alpha_{e}, \mathrm{C}_{0}\right)$ finite, $c=c\left(\delta, \mathrm{C}_{\mathrm{P}}, \mathrm{C}_{\mathrm{D}}, \alpha_{e}, C_{0}\right)>0, u(\cdot)$ as in Lemma 4.4 and $T^{\prime} \in\left[T-(R / 2)^{2}+r^{2}, T\right]$,

$$
\frac{\boldsymbol{\pi}\left(Q\left(T^{\prime}-r^{2}, T^{\prime} ; z, R\right) \cap u^{-1}([1, \infty))\right)}{\boldsymbol{\pi}\left(Q\left(T^{\prime}-r^{2}, T^{\prime} ; z, r\right)\right)} \geq \delta \quad \Rightarrow \quad u\left(T-4 R^{2}, z\right) \geq c\left(\frac{\boldsymbol{\pi}\left(Q\left(T^{\prime}-r^{2}, T^{\prime} ; z, r\right)\right)}{\boldsymbol{\pi}\left(Q\left(T-R^{2}, T ; z, R\right)\right)}\right)^{\theta} .
$$

Proof of Theorem 1.6 [sketch, following [Gr] ]. In case of manifolds, the derivation of PHI from the first and second growth lemmas is standard in the literature. We sketch here the adaptation for discrete graphs of the argument provided near the end of [Gr, Section 4], where one is restricted to choose cylinders of radii at least $R_{0} \geq 1$. Specifically, with $Q(R)$ denoting the cylinder $Q(T-$ $\left.R^{2}, T ; z, R\right)$, our goal is to show that for some $\gamma\left(\mathrm{C}_{\mathrm{P}}, \mathrm{C}_{\mathrm{D}}, \alpha_{e}, \mathrm{C}_{0}\right)>0$ if $\sup _{Q\left(T-4 R^{2}, T-3 R^{2} ; z, R\right)} u=1$ for some positive solution $u(\cdot, \cdot)$ on $Q(8 R)$, then necessarily $u\left(T-48 R^{2}, z\right) \geq \gamma$. To this end, for $R_{0} \geq 1$ of Lemma 4.5 set $\eta_{0}:=\mathrm{C}_{\mathrm{D}}^{-R_{0}} /\left(\mathrm{C}_{0} R_{0}^{2}\right)$ and fix the largest $\eta \in\left(0, \eta_{0}\right]$ for which Lemma 4.5 holds. By Lemma 4.4 if $\boldsymbol{\pi}\left(E_{0}\right) \geq \delta \boldsymbol{\pi}(Q(4 R))$ for $E_{0}:=Q(4 R) \cap u^{-1}\left(\left[2^{-1}, \infty\right)\right.$ and $\delta:=\eta /\left(64 \mathrm{C}_{0} \mathrm{C}_{\mathrm{D}}^{4}\right)$, then $u\left(T-48 R^{2}, z\right) \geq \varepsilon$ for some $\varepsilon=\varepsilon\left(\delta, \mathrm{C}_{\mathrm{P}}, \mathrm{C}_{\mathrm{D}}, \alpha_{e}, \mathrm{C}_{0}\right)>0$, verifying the claimed PHI in this case. Next, suppose to the contrary that $\delta \boldsymbol{\pi}(Q(4 R))>\boldsymbol{\pi}\left(E_{0}\right)$. Recall our assumption that $u\left(T-t_{0}, x_{0}\right)=$ 1 for some $t_{0} \in\left[3 R^{2}, 4 R^{2}\right]$ and $x_{0} \in \mathbb{B}(z, R)$, whereas by the uniform volume doubling condition and our choice of $\delta$,

$$
\eta \boldsymbol{\pi}\left(Q\left(T-t_{0}-(R / 2)^{2}, T-t_{0} ; x_{0}, R / 2\right)\right) \geq \delta \boldsymbol{\pi}(Q(4 R))>\boldsymbol{\pi}\left(E_{0}\right) .
$$

Next let $r_{0}$ be the maximum integer $r \leq R / 2$ for which

$$
\boldsymbol{\pi}\left(Q\left(T-t_{0}-r^{2}, T-t_{0} ; x_{0}, r\right) \cap u^{-1}\left(\left[2^{-1}, \infty\right)\right)\right) \geq \eta \pi\left(Q\left(T-t_{0}-r^{2}, T-t_{0} ; x_{0}, r\right)\right) .
$$


Having $u\left(T-t_{0}, x_{0}\right)=1$, we deduce from the uniform volume doubling condition and our choice of $\eta \leq \eta_{0}$ that necessarily $r_{0} \geq R_{0}$. Further, in view of (4.18) also $r_{0}<R / 2$ and employing Lemma 4.5. we have that

$$
u\left(T-t_{1}, x_{1}\right)=\sup _{Q\left(T-t_{0}-2\left(r_{0}+1\right)^{2}, T-t_{0} ; x_{0}, r_{0}+1\right)} u \geq 2 .
$$

This procedure is iterated in the same way as at [Gr, end of Section 4], with the only the change being that $r_{k}$ is defined be the maximum integer $r$ such that

$$
\frac{\boldsymbol{\pi}\left(Q\left(T-t_{k}-r^{2}, T-t_{k} ; x_{k}, r\right) \cap u^{-1}\left(\left[2^{k-1}, \infty\right)\right)\right)}{\boldsymbol{\pi}\left(Q\left(T-t_{k}-r^{2}, T-t_{k} ; x_{k}, r\right)\right)} \geq \eta
$$

The proof concludes as in $\mathrm{Gr}$ by choosing a good index $k$ and applying Lemma 4.7 to the pair of cylinders $Q\left(T-t_{k}-r_{k}^{2}, T-t_{k} ; x_{k}, r_{k}\right)$ and $Q(4 R)$.

Proof of Proposition 1.7. Fixing $y_{j} \in \mathbb{B}(z, R)$ and $\left(T-s_{j}\right) \in\left[R^{2}, 4 R^{2}\right]$ such that $s_{2} \geq s_{1}$, we consider nested time-space cylinders $Q(i):=\left[s_{1}, s_{1}+R_{i}^{2}\right] \times \mathbb{B}\left(y_{1}, R_{i}\right)$ for $R_{i}=2^{i}, i \geq 0$ and the corresponding $M(i):=\sup _{Q(i)}\{u\}, m(i):=\inf _{Q(i)}\{u\}$ and $w(i):=M(i)-m(i)$. With $i_{2}:=\sup \{i$ : $Q(i) \subseteq Q\}$ we have that $w\left(i_{2}\right) \leq M\left(i_{2}\right) \leq \sup _{Q}\{u\}$. Similarly, setting $r:=\left|s_{2}-s_{1}\right|^{1 / 2} \vee d\left(y_{1}, y_{2}\right)$ and $i_{1}:=\inf \left\{i: r \leq R_{i}\right\}$ we have that $\left(s_{j}, y_{j}\right) \in Q\left(i_{1}\right), j=1,2$, hence $\left|u\left(s_{2}, y_{2}\right)-u\left(s_{1}, y_{1}\right)\right| \leq w\left(i_{1}\right)$. Clearly $R_{i_{1}} \leq 2 r$, while $R \leq 2 R_{i_{2}}$ (since $\left(T-s_{1}\right) \geq R^{2}$ and $\mathbb{B}\left(y_{1}, R\right) \subseteq \mathbb{B}(z, 2 R)$ ). The inequality (1.25) is trivial unless $r \leq R / 4$ and thereby $i_{1} \leq i_{2}$. It thus suffices to show that $w(i-1) \leq$ $(1-\gamma) w(i)$ for some $\gamma\left(\theta_{j}\right)$ from (1.24) and all $i \leq i_{2}$ (as then $w\left(i_{1}\right) \leq(1-\gamma)^{i_{2}-i_{1}} w\left(i_{2}\right)$, yielding (1.25) for $\left.2^{-h}=1-\gamma\right)$. To this end, consider for (non-negative) solutions $u-m(i)$ and $M(i)-u$, the PHI in $Q(i) \subseteq Q$ with $\theta_{1}=\theta_{2}=\frac{1}{\sqrt{2}}, \theta_{3}=\frac{\sqrt{3}}{2}$ and $\theta_{4}=1$, to compare the solution at $\left(s_{1}+2 R_{i-1}^{2}, y_{1}\right)$ with its infimum over $Q(i-1)$. Setting $v(i)=u\left(s_{1}+2 R_{i-1}^{2}, y_{1}\right)$, these comparisons yield by the PHI that

$$
\begin{gathered}
\gamma(v(i)-m(i)) \leq m(i-1)-m(i) \\
\gamma(M(i)-v(i)) \leq M(i)-M(i-1),
\end{gathered}
$$

and hence $w(i-1) \leq(1-\gamma) w(i)$, as claimed.

\section{From Ghiu and Phi to Gaussian LOWER Bounds}

In this section we establish the matching GHKE of (1.28) out of the (weaker) upper bound (1.27) and the PHI (1.24). To this end, we start with the following elementary fact.

Lemma 5.1. Suppose $\Gamma_{\tau}: V \mapsto \mathbb{R}_{+}$are such that

$$
\Gamma_{\tau}(z) \leq \frac{C}{v(\sqrt{\tau})} e^{-\rho_{\tau}(x, z) / C}
$$

for some $C<\infty, x \in V$, a doubling function $v(r) \geq \mathrm{C}_{\mathrm{v}}^{-2}|\mathbb{B}(x, r)|$ and

$$
\rho_{t}(x, z):=d(x, z)\left(\frac{d(x, z)}{t} \wedge 1\right), \quad x, z \in V, t \geq 0 .
$$

Then, for some $c^{\prime}\left(\mathrm{C}_{\mathrm{v}}, C\right)$ finite,

$$
\Gamma_{\tau}\left(\mathbb{B}(x, R)^{c}\right) \leq c^{\prime} e^{-R /(2 C)}, \quad \forall R \geq \tau \geq 1, \quad \forall x \in V .
$$

Further, if $\Gamma_{\tau}$ are probability measures, then for $b>0$ and some $\kappa\left(\mathrm{C}_{\mathrm{v}}, C, b\right)<\infty$,

$$
\inf _{\tau \geq b, x \in V}\left\{\Gamma_{\tau}(\mathbb{B}(x, \kappa \sqrt{\tau}))\right\} \geq \frac{1}{2} .
$$


Proof. Note that $1-e^{-(1+2(\ell \wedge \tau)) /(C \tau)} \leq 3 \ell /(C \tau)$ for $\ell \geq 1$. From (5.1) we thus get after summation by parts that

$$
\Gamma_{\tau}\left(\mathbb{B}(x, R)^{c}\right) \leq \frac{C}{v(\sqrt{\tau})} \sum_{\ell>R}|\partial \mathbb{B}(x, \ell)| e^{-\ell(\ell \wedge \tau) /(C \tau)} \leq 3 C_{v}^{2} \sum_{\ell>R} \frac{v(\ell) \ell}{v(\sqrt{\tau}) \tau} e^{-\ell(\ell \wedge \tau) /(C \tau)} .
$$

With $v(\ell)$ doubling, one has $v(\ell) \leq v(\sqrt{\tau})(2 \ell / \sqrt{\tau})^{c_{v}}$ for $c_{v}:=\log _{2} C_{v}$ and any $\ell \geq \sqrt{\tau}$. Hence, for some $c^{\prime}\left(\mathrm{C}_{\mathrm{v}}, C\right)$ and any $R \geq \tau \geq 1$,

$$
\Gamma_{\tau}\left(\mathbb{B}(x, R)^{c}\right) \leq 3 \mathrm{C}_{\mathrm{v}}^{2} \int_{R}^{\infty}(4 u)^{c_{v}+1} e^{-u / C} d u \leq c^{\prime} e^{-R /(2 C)}
$$

as claimed in (5.3). Similarly, for $\kappa=\kappa\left(\mathrm{C}_{\mathrm{v}}, C, b\right)$ large enough and all $\tau \geq b$,

$$
\Gamma_{\tau}\left(\mathbb{B}(x, \kappa \sqrt{\tau})^{c}\right) \leq 3 \mathrm{C}_{\vee}^{2} \int_{\kappa}^{\infty}(4 u)^{c_{v}+1} e^{-u(u \wedge \sqrt{b}) / C} d u \leq \frac{1}{2},
$$

yielding (5.4) in case $\Gamma_{\tau}(V)=1$.

We next utilize the fact that for $\left\{K_{t}\right\}$ of (1.1), the transition probabilities $K_{\cdot, t}(\cdot, y)$ of the CSRW and DTRW, are solutions of (1.23).

Lemma 5.2. Fixing Borel measurable $\left\{\boldsymbol{\Pi}_{s}\right\}, t \geq 0$ and $z_{\star} \in V$, the functions $u(s, x):=K_{s, t}\left(x, z_{\star}\right)$ for the CSRW and DTRW associated with (1.1), solve the corresponding heat equation (1.23) on the time-space cylinder $Q(0, t ; z, R)$ for any $R \geq 1$ and $z \in V$. The PHI then implies that:

(a). For some $\gamma(\varphi, \delta) \in(0,1)$, any $\delta \in(0,1), \varphi \geq 1 /(1-\delta)$, all $t \geq \tau \geq 1$, and $x_{1}, x_{2}, z_{\star} \in V$,

$$
d\left(x_{1}, x_{2}\right) \leq 2[2 \varphi \sqrt{\tau}] \quad \Longrightarrow \quad K_{t-\tau, t}\left(x_{2}, z_{\star}\right) \geq \gamma K_{t-\delta \tau, t}\left(x_{1}, z_{\star}\right),
$$

where for DTRW we further assume that $\tau \in \mathbb{N}$ and $d\left(x_{1}, x_{2}\right) \leq(1-\delta) \tau \in \mathbb{N}$.

(b). Suppose in addition that $\mathrm{C}_{0}:=\sup _{s, x}\left\{\frac{\pi_{s}(x)}{\pi_{0}(x)}\right\}$ is finite, $s \mapsto \pi_{s}(\cdot)$ is non-decreasing and the DTRW is uniformly lazy. Then,

$$
s \mapsto D_{s, t}(y):=\sum_{x \in V} \pi_{s}(x) K_{s, t}(x, y)^{2}
$$

is non-decreasing on $[0, t]$. Further, for $C_{1}=C_{1}\left(\gamma, \alpha_{l}, C_{0}\right)$ finite,

$$
C_{1} D_{t-2 s, t}(y) \geq D_{t-s, t}(y), \quad \forall y \in V, s \geq 0 .
$$

Remark 5.3. From part (b) we have that the non-decreasing $\bar{D}(s):=\pi_{t}(y) / D_{t-s, t}(y)$ is a doubling function, with $\bar{D}(0)=1$ such that $\bar{D}(2 s) \leq C_{1} \bar{D}(s)$ for all $y \in V$ and $2 s \leq t$.

Proof. (a). From (1.3) it immediately follows that for DTRW the non-negative $u(k, x)=K_{k, t}\left(x, z_{\star}\right)$ satisfies (1.23) on $Q(0, t ; z, R)$ of (1.22) (with $\left.\partial_{-s} u(s, \cdot)=u(s-1, \cdot)-u(s, \cdot)\right)$. Similarly, in case of CSRW, it follows from (1.5) that $s \mapsto u(s, x)=K_{s, t}\left(x, z_{\star}\right)$ is an absolutely continuous, solution of (1.23) on $Q(0, t ; z, R)$. Next, if $d\left(x_{1}, x_{2}\right) \leq 2 R$ for integer $R:=[2 \varphi \sqrt{\tau}] \geq 1$, then $x_{1}, x_{2} \in \mathbb{B}(z, R)$ for some $z \in V$. Further, for $\tau \geq 1$ we have $R+1 \geq 2 \varphi \sqrt{\tau} \geq 2 /(1-\delta)$, hence the PHI applies for $T=t, \tau_{2}=\tau$ and $\tau_{1}=\delta \tau$, with $\varphi \theta_{1}=\sqrt{\delta} / 2, \varphi \theta_{2}=\sqrt{\delta} /(1+\delta)<1 / 2, \varphi \theta_{3}=1 / 2, \varphi \theta_{4}=1 /(1+\delta)$ and the corresponding $\gamma=\gamma\left(\theta_{i}\right) \in(0,1)$.

(b). While proving Lemma 3.5, we showed that $s \mapsto E_{s}(u)$ of (3.36) is non-decreasing whenever $u_{s}(x) \geq 0$ solves (1.23) for some $u_{t} \in \mathcal{C}_{0}(V)$. By part (a) this applies to $s \mapsto D_{s, t}(y)$ which corresponds to $u_{s}(x)=K_{s, t}(x, y)$ and $f_{s}(x) \equiv 1$. Next, from (5.5) for $x_{1}=x_{2}$ we have that $K_{t-2 s, t}(x, y) \geq \gamma K_{t-s, t}(x, y)$ for $\gamma(2,1 / 2)>0$, all $x, y \in V, 2 s \in[1, t]$, yielding that $C_{1} D_{t-2 s, t}(y) \geq$ $D_{t-s, t}(y)$ for $C_{1}=\mathrm{C}_{0} \gamma^{-2}$ finite. Lowering $\gamma \leq \alpha_{l}$ for lazy DTRW, or $\gamma \leq e^{-1}$ for CSRW, we further have that $K_{t-1, t}(y, y) \geq \gamma$, hence $C_{1} D_{t-1, t}(y) \geq D_{t, t}(y)$, extending (5.7) to all $2 s \in[0, t]$. 
Utilizing the GHKU of Theorem [1.8(a) as well as Lemmas [3.6, 5.1 and 5.2(b), we next establish a moment generating bound which is key for getting matching GHKE.

Lemma 5.4. In the setting of Theorem 1.8(b), for dynamics (1.3) and (1.5), the functions $\rho_{t}(\cdot)$ of (5.2) and $D_{s, t}(\cdot)$ of (5.6), some $\theta_{0}\left(\gamma, \mathrm{C}_{\mathrm{v}}, \alpha_{l}\right)>0$ and $C_{2}\left(\gamma, \mathrm{C}_{\mathrm{v}}, \alpha_{l}\right)$ finite,

$$
D_{\tau, 2 \tau}\left(y ; \theta_{0}\right):=\sum_{z \in V} \pi_{\tau}(z) K_{\tau, 2 \tau}(z, y)^{2} e^{\theta_{0} \rho_{\tau}(z, y)} \leq C_{2} D_{\tau, 2 \tau}(y), \quad \forall y \in V, \tau \geq 1 .
$$

Proof. Fixing $y \in V, \tau \geq 1$ and $t=2 \tau$, let

$$
J(\ell, s):=\sum_{z \in V} \pi_{t-s}(z) K_{t-s, t}(z, y)^{2} \mathbf{1}_{\{d(z, y) \geq \ell\}}, \quad \ell \geq 0, s \in[0, t] .
$$

Since $\partial_{-\ell}\left\{\ell\left(\frac{\ell}{\tau} \wedge 1\right)\right\} \geq-2 \ell / \tau$ and $1-e^{w} \leq-w$, it follows after summation by parts that

$$
D_{\tau, 2 \tau}(y ; \theta)=\sum_{\ell=0}^{\infty} e^{\theta \ell\left(\frac{\ell}{\tau} \wedge 1\right)}(J(\ell, \tau)-J(\ell+1, \tau)) \leq J(0, \tau)+2 \theta \sum_{\ell=1}^{\infty} J(\ell, \tau) \frac{\ell}{\tau} e^{\theta \ell\left(\frac{\ell}{\tau} \wedge 1\right)} .
$$

With $J(0, \tau)=D_{\tau, 2 \tau}(y)$ we thus get (5.8) upon showing that

$$
J(\ell, \tau) \leq C_{2}^{\prime} J(0, \tau) e^{-2 \theta_{0} \ell\left(\frac{\ell}{\tau} \wedge 1\right)},
$$

for some $\theta_{0}\left(\gamma, \mathrm{C}_{\mathrm{v}}, \alpha_{l}\right)$ positive, $C_{2}^{\prime}\left(\gamma, \mathrm{C}_{\mathrm{v}}, \alpha_{l}\right)$ finite and all $\ell \geq \ell_{0}\left(\gamma, \mathrm{C}_{\mathrm{v}}, \alpha_{l}\right)$ finite, whereupon taking $C_{2}^{\prime} \geq e^{2 \kappa \theta_{0}}$ it suffices to show (5.10) for $\ell>\sqrt{\kappa \tau}$. To this end, we proceed by adapting the proof of [CGZ, Prop. 5.4] to handle both time-varying $\pi_{t}(\cdot)$ and the CSRW (see also [Fo, Lemma 4.1] for CSRW with constant conductances). First apply Lemma 3.6 for $u_{s}(\cdot):=K_{t-s^{\prime}+s, t}(\cdot, y)$, with $u_{s^{\prime}} \in \mathcal{C}_{0}(V)$, and the Lipschitz function $\rho\left(\cdot ; \ell^{\prime}\right):=\ell^{\prime}+1-d(\cdot, y) \wedge \ell^{\prime}$, to deduce that for some $\eta=\eta\left(\alpha_{l}\right)>0$, any $s^{\prime} \in[0, t], \ell^{\prime} \geq 0$ and $y \in V$,

$$
s \mapsto E_{s}\left(\ell^{\prime}, s^{\prime}\right):=\sum_{z \in V} \pi_{t-s^{\prime}+s}(z) K_{t-s^{\prime}+s, t}(z, y)^{2} e^{-\eta(s+1) I\left(\rho\left(z ; \ell^{\prime}\right) /(s+1)\right)},
$$

is non-decreasing on $\left[0, s^{\prime}\right]$. Further, $I(1)=1$ and $\rho\left(z ; \ell^{\prime}\right)=1$ whenever $d(z, y) \geq \ell^{\prime}$ while $\rho\left(z ; \ell^{\prime}\right) \geq$ $\ell^{\prime}-\ell$ whenever $d(z, y)<\ell \leq \ell^{\prime}$. Hence, for any $s \in\left[0, s^{\prime}\right]$ and $\ell \leq \ell^{\prime}$,

$$
e^{-\eta} J\left(\ell^{\prime}, s^{\prime}\right) \leq E_{0}\left(\ell^{\prime}, s^{\prime}\right) \leq E_{s^{\prime}-s}\left(\ell^{\prime}, s^{\prime}\right) \leq J(\ell, s)+J(0, s) e^{-\eta\left(s^{\prime}-s+1\right) I\left(\frac{\ell^{\prime}-\ell}{s^{\prime}-s+1}\right)} .
$$

We then get (5.10) upon recalling Remark 5.3 that due to the PHI the function $s \mapsto \bar{D}(s)=$ $J(0,0) / J(0, s)$ is non-decreasing and $C_{1}$-doubling, hence regular in the sense of [CGZ, Def. 5.1]. Indeed, CGZ, (5.11)] is derived from [CGZ, (5.10)] by iterating (5.11) for consecutive terms of the sequence $\ell_{j}=\ell / 2+\ell /(j+1), s_{j}=\tau 2^{-(j-1)}$, starting at $(\ell, \tau)$ when $j=1$, and stopping at $j_{0}:=\min \left\{j \geq 1: \ell_{j}>s_{j}\right\}$ (since in their case, of DTRW, one has that $J(R, s)=0$ whenever $R>s$ ). For such parameters $\left(\ell^{\prime}-\ell\right) /\left(s^{\prime}-s+1\right) \leq \ell^{\prime} / s^{\prime} \leq 1$, hence $I(r)=r^{2}$ (even for CSRW), and taking $\kappa \geq \theta_{0}^{-1}\left(\log \left(2 C_{1}\right)+\eta\right)$ makes [CGZ, CASE 1] hold here as well. Thus, the only difference is that for the CSRW we still have to bound the last term of the iteration, $e^{j_{0} \eta} J\left(\ell_{j_{0}}, s_{j_{0}}\right)$, by the RHS of (5.10). For this task apply Lemma 5.1 to $\Gamma_{s}(z):=\pi_{t-s}(z) K_{t-s, t}(z, y)^{2}$, in which case (5.3) amounts to

$$
J(R, s) \leq c^{\prime} e^{-R / C}, \quad \forall R \geq s \geq 1
$$

(for $C$ of (1.27) and $c^{\prime}\left(\mathrm{C}_{\mathrm{v}}, C\right)$ finite). Next, recall (5.7) that $J(0, \tau) \geq\left(C_{1} \mathrm{C}_{\mathrm{v}}\right)^{-1} \tau^{-c_{1}}$ for some $c_{1}$ finite and all $\tau \geq 1$. With $\ell_{j_{0}} \geq s_{j_{0}} \vee \ell / 2$ and $e^{j_{0} \eta} \leq \tau^{\eta / \log 2}$ (since $s_{j_{0}} \geq 1$ ), it thus follows that for $c_{1}^{\prime}=c_{1}+\eta / \log 2$ and some $C_{1}^{\prime}$ finite,

$$
e^{j_{0} \eta} J\left(\ell_{j_{0}}, s_{j_{0}}\right) \leq C_{1}^{\prime} J(0, \tau) \tau^{c_{1}^{\prime}} e^{-\ell /(2 C)},
$$


which for $\ell \geq \sqrt{\tau}$ and $\theta_{0} \leq(5 C)^{-1}$, is further bounded by the RHS of (5.10).

Proof of Theorem $1.8(b)$. Proceeding to derive the matching GHKE of (1.28), since our assumptions apply for $\left.\left(K_{s+r}, \pi_{s+r}\right): r \in[0, t-s]\right\}$, it suffices to do so for $s=0$ and fixed $x, y \in V$ such that $d(x, y) \leq t$.

- Step I: Improved GHKU. Recall the GHKU (1.27) implying that (5.1) holds for $K_{s, s+\tau}(x, \cdot)$ with $C<\infty$ independent of $s, \tau \geq 0$ and $x \in V$. Further, by the triangle inequality, we have for the non-increasing $t \mapsto \rho_{t}(x, z)$ of (5.2) that

$$
\frac{1}{2} \rho_{t}(x, y) \leq \rho_{t}(x, z)+\rho_{t}(y, z), \quad \forall z \in V, t \geq 0 .
$$

Hence, setting $t=2 \tau, \tau \geq 1$ and $\theta_{1}=\frac{1}{2}\left(\theta_{0} \wedge C^{-1}\right)$, by Chapman-Kolmogorov and (5.12), followed by Cauchy-Schwartz, (5.8), Lemma [5.4] and the inequality (5.7), we arrive at

$$
\begin{aligned}
K_{0, t}(x, y)^{2} e^{\theta_{1} \rho_{t}(x, y)} & \leq\left[\sum_{z \in V} K_{0, \tau}(x, z) e^{\theta_{1} \rho_{t}(x, z)} K_{\tau, t}(z, y) e^{\theta_{1} \rho_{t}(y, z)}\right]^{2} \\
& \leq \mathrm{C}_{\mathrm{v}} D_{\tau, t}\left(y ; 2 \theta_{1}\right) \sum_{z \in V} K_{0, \tau}(x, z)^{2} e^{2 \theta_{1} \rho_{\tau}(x, z)} \\
& \leq \mathrm{C}_{\mathrm{v}} C_{2} D_{\tau, t}(y) \frac{C}{v(\sqrt{\tau})} \sum_{z \in V} K_{0, \tau}(x, z) \leq \frac{C^{\prime}}{v(\sqrt{t})} D_{0, t}(y)
\end{aligned}
$$

where $C^{\prime}=\mathrm{C}_{\mathrm{v}}^{2} C_{2} C_{1} C$. Applying the same argument on $[\tau, 2 \tau]$ instead of $[0, t]$, yields that for any $y \in V$ and $\tau \geq 1$,

$$
\Gamma_{\tau}(z):=\frac{\pi_{\tau}(z) K_{\tau, 2 \tau}(z, y)^{2}}{D_{\tau, 2 \tau}(y)} \leq \frac{\mathrm{C}_{\mathrm{v}} C^{\prime}}{v(\sqrt{\tau})} e^{-\theta_{1} \rho_{\tau}(z, y)} .
$$

In view of (5.6), these $\Gamma_{\tau}(\cdot)$ are probability measures on $V$, hence by Lemma 5.1 there exists $\kappa\left(\mathrm{C}_{\mathrm{v}}, \mathrm{C}_{\mathrm{v}} C^{\prime} \vee \theta_{1}^{-1}, 1\right)$ finite such that for $R=\kappa \sqrt{\tau}$,

$$
\Gamma_{\tau}(\mathbb{B}(y, R)) D_{\tau, t}(y) \geq \frac{1}{2} D_{\tau, t}(y) \geq \frac{1}{2 C_{1}} D_{0, t}(y)
$$

(using the RHS of (5.7) for the last inequality). By the definition (1.8) and Lemma 5.2(a) (at $x_{1}=z$, $\left.x_{2}=x, z_{\star}=y\right)$, we have for $\gamma(\kappa / 2,1 / 2)>0$ and $R$ as above,

$$
\left[\frac{\mu_{0, t}(y)}{\pi_{0}(\mathbb{B}(y, R))}\right]^{2} \geq \inf _{x \in \mathbb{B}(y, R)}\left\{K_{0, t}(x, y)^{2}\right\} \geq \gamma^{2} D_{\tau, t}(y) \frac{\Gamma_{\tau}(\mathbb{B}(y, R))}{\pi_{\tau}(\mathbb{B}(y, R))}
$$

(where for DTRW we restrict to $\tau \geq 2 R$ ). In view of the assumed uniform volume growth with $v(r)$ doubling, from (5.14) and (5.15) it follows that for some $C_{3}\left(\mathrm{C}_{\mathrm{v}}, C_{1}, \kappa\right)$ finite,

$$
\mu_{0, t}(y)^{2} \geq \frac{\gamma^{2}}{2 C_{1} C_{\mathrm{v}}^{3}} v(R) D_{0, t}(y) \geq C_{3}^{-1} v(\sqrt{t}) D_{0, t}(y) .
$$

For DTRW, our derivation of (5.16) required $t \geq 2(2 \kappa)^{2}$, but with $K_{t}(\cdot, \cdot)$ uniformly elliptic, one easily extends (5.16) to all $t \geq 0$ upon increasing $C_{3}$ to some $C_{3}(\bar{\alpha})$ finite. Finally, combining (5.13) and (5.16) we have that for $C_{\star}:=\sqrt{C_{3} C^{\prime}} \vee\left(2 / \theta_{1}\right)$ finite,

$$
K_{0, t}(x, y) \leq \frac{C_{\star} \mu_{0, t}(y)}{v(\sqrt{t})} e^{-\rho_{t}(x, y) / C_{\star}},
$$

as stated in the RHS of (1.28).

- Step II: matching GHKL. With (5.17) holding for $K_{s, s+t}(\cdot, \cdot), s \geq 0$, it yields the bound (5.4) for $b=1 / 2$, the probability measures $K_{s, s+\tau}(x, \cdot)$ and $\Gamma_{\tau}(\cdot):=\pi_{s}(\cdot) K_{s, s+\tau}(\cdot, y) / \mu_{s, s+\tau}(y)$, some 
$\kappa\left(\mathrm{C}_{\mathrm{v}}, C, b\right) \geq 2$, all $x, y \in V, s \geq 0$ and $\tau \geq b$. Fixing $\varphi \geq 2\left(1+\kappa^{2}\right), \delta=1 / 2$ and $\gamma \in(0,1)$ as in (5.5), we further have that for all $x, y \in V, t \geq \tau \geq 1$ and $r \leq 2 \varphi \sqrt{\tau}$ (with $4 r \leq \tau \in \mathbb{N}$ in case of DTRW),

$$
K_{t-\tau, t}(x, y) \geq \gamma \sup _{z \in \mathbb{B}(x, 2 r)}\left\{K_{t-\delta \tau, t}(z, y)\right\}
$$

Setting $n_{\star}=1$ for CSRW and $n_{\star}=\left\lceil(8 \varphi)^{2}\right\rceil$ for DTRW, (5.18) applies when $r=[2 \varphi \sqrt{\tau}]$ and $\tau \geq n_{\star}$. Further, if $d(x, y) \leq r$ then $\mathbb{B}(y, r) \subseteq \mathbb{B}(x, 2 r)$, so $r=[2 \varphi \sqrt{\tau}] \geq \kappa \sqrt{\tau}$ yields by (5.4)

$$
K_{t-\tau, t}(x, y) \geq \frac{\gamma}{\pi_{t-\delta \tau}(\mathbb{B}(y, r))} \sum_{z \in \mathbb{B}(y, r)} \pi_{t-\delta \tau}(z) K_{t-\delta \tau, t}(z, y) \geq \frac{\gamma \mu_{t-\delta \tau, t}(y)}{2 \mathrm{C}_{v} v(2 \varphi \sqrt{\tau})} .
$$

With $v(\cdot)$ volume doubling and $s \mapsto \mu_{s, t}(y)$ is non-decreasing, taking $\tau=t \geq n_{\star}$ in (5.19) yields the GHKL for near-diagonal $d(x, y) \leq 2 \varphi \sqrt{t}$. It extends to all $d(x, y) \leq t<n_{\star}$ since only $y=x$ is relevant for $t<1$ (and the GHKL then trivially holds), and for DTRW having uniformly elliptic conductances implies that $K_{0, t}(x, y) \geq\left(\alpha_{e}\right)^{n_{\star}}$ whenever $d(x, y) \leq t<n_{\star}$.

Considering hereafter $d(x, y) \in[2 \varphi \sqrt{t}, t]$ and $t \geq n_{\star}$, fix integers $R=[2 \varphi t / d(x, y)] \geq 4$ and $\ell=\lceil d(x, y) / R\rceil \geq 4$. We further find $x_{i} \in V$ with $x_{0}=x$ and $x_{\ell}=y$ such that $d\left(x_{i}, x_{i+1}\right) \leq R$ for $0 \leq i \leq \ell-1$. Setting $\tau=t / \ell \geq 2$ (or its integer part for the DTRW), let $t_{0}=0, t_{2 \ell}=t$ and $t_{2 i-1}:=\left(t_{2 i}+t_{2(i-1)}\right) / 2$, with $t_{2 i}-t_{2(i-1)}=\tau$ for CSRW, or in $\{\tau, \tau+1\}$ for DTRW (as needed). It is easy to check that $\kappa \sqrt{\tau} \leq R \leq 2 \varphi \sqrt{\tau}$, and further that the extra requirement $4 R \leq \tau \in \mathbb{N}$ which we need in case of the DTRW, holds whenever $d(x, y) \leq t / 6$. For such $x, y, t$ we get by Chapman-Kolmogorov and (5.18) followed by (5.4), that

$$
\begin{aligned}
& K_{0, t}(x, y) \geq \sum_{\left\{z_{i} \in \mathbb{B}\left(x_{i}, 2 R\right)\right\}} K_{0, t_{2}}\left(x, z_{1}\right)\left[\prod_{i=2}^{\ell-1} K_{t_{2(i-1)}, t_{2 i}}\left(z_{i-1}, z_{i}\right)\right] K_{t_{2(\ell-1)}, t}\left(z_{\ell-1}, y\right) \\
& \geq \gamma^{\ell-1}\left[\sum_{z \in \mathbb{B}(x, R)} K_{0, t_{2}}(x, z)\right] \prod_{i=2}^{\ell-1}\left[\sum_{z \in \mathbb{B}\left(x_{i-1}, R\right)} K_{t_{2 i-1}, t_{2 i}}\left(x_{i-1}, z\right)\right] K_{t_{2 \ell-1}, t}\left(x_{\ell-1}, y\right) \\
& \geq\left(\frac{\gamma}{2}\right)^{\ell-1} K_{t_{2 \ell-1}, t}\left(x_{\ell-1}, y\right) \geq\left(\frac{\gamma}{2}\right)^{\ell} \frac{\mu_{t-\delta \tau^{\prime}, t}(y)}{\mathrm{C}_{\mathrm{v}} v\left(2 \varphi \sqrt{\tau^{\prime}}\right)}
\end{aligned}
$$

where $d\left(x_{\ell-1}, y\right) \leq R$ so the last inequality is merely (5.19) for $\tau^{\prime}:=t-t_{2 \ell-1} \in[\tau / 2, \tau]$ and $\kappa \sqrt{\tau^{\prime}} \leq R \leq 2 \varphi \sqrt{\tau^{\prime}}$. Consequently, with $\tau \leq t, v(\cdot)$ volume doubling and $\ell \geq d(x, y)^{2} /(2 \varphi t)$,

$$
K_{0, t}(x, y) \geq\left(\frac{\gamma}{2}\right)^{\ell} \frac{\mu_{0, t}(y)}{\mathrm{C}_{\mathrm{v}} v(2 \varphi \sqrt{t})} \geq \frac{\mu_{0, t}(y)}{C v(\sqrt{t})} \exp \left\{-C d(x, y)^{2} / t\right\},
$$

for some $C=C\left(\mathrm{C}_{\mathrm{v}}, \gamma, \varphi\right)$ finite. Note that for $d(x, y) / t \in[1 / 6,1]$ and uniformly elliptic DTRW we have that $K_{0, t}(x, y) \geq\left(\alpha_{e}\right)^{t} \geq e^{-C d(x, y)^{2} / t}$, where $C=1 \vee 36 \log \left(1 / \alpha_{e}\right)$. Recalling that $\mu_{0, t}(y) \leq$ $1 \leq C v(\sqrt{t})$ this extends the validity of (5.20) to all $d(x, y) \leq t$.

\section{The Perturbative Regime}

For $\left\{K_{t}\right\}$ of (1.1) the transition kernels $\left\{K_{s, t}\right\}$ are unchanged by re-scaling the conductances

$$
\widehat{\pi}_{u ; v}(x, y)=e^{a_{u}-a_{v}} \pi_{u}(x, y) \quad \forall(x, y) \in E
$$

In particular, for $\left\{a_{t}\right\}$ of (1.31) one has that $a_{u^{\prime}}-a_{u} \geq \rho_{\boldsymbol{\pi}}\left(u^{\prime}, u\right)$ for all $u^{\prime} \geq u$ and hence $u \mapsto \widehat{\pi}_{u ; v}(x)$ is non-decreasing (for each $x \in V$ ). More generally, working under the framework of Example 2.3, 
the Nash profiles re-scale as

$$
\mathcal{N}_{Q_{u}, \widehat{\pi}_{u ; v}}(\mathrm{~s})=\mathcal{N}_{Q_{u}, \pi_{u}}\left(e^{a_{v}-a_{u}} \mathrm{~s}\right),
$$

yielding an on-diagonal transition density upper bound when $u \mapsto \widehat{\pi}_{u ; v}(x)$ are non-decreasing and (1.30) holds. Also, if (1.30) applies for $a_{t}$ of (1.31), then $\mu_{s, t}(\cdot)$ of (1.8) are uniformly bounded below provided $s / t$ is.

Lemma 6.1. Consider $\left\{K_{t}\right\}$ of (1.1) with non-decreasing $u \mapsto \widehat{\pi}_{u ; v}(x)$ of (6.1), such that the non-decreasing $t \mapsto a_{t}$ satisfies (1.30).

(a). Suppose $c_{\star}:=\inf _{t, x}\left\{\pi_{t}(x)\right\}>0$ and for some finite $\mathrm{C}_{\mathrm{n}}, \mathrm{s}_{0}$ and non-decreasing $N(\cdot)$,

$$
N(\mathrm{~s}) \geq \sup _{t}\left\{\mathcal{N}_{K_{t}^{2}, \pi_{t}}(\mathrm{~s})\right\}, \quad \inf _{\mathrm{s} \geq \mathrm{s}_{0}}\left\{\frac{N\left(\mathrm{C}_{\mathrm{n}} \mathrm{s}\right)}{N(\mathrm{~s})}\right\} \geq 2 .
$$

Then, for the dynamic (1.3), $\psi(\mathrm{t})=1 / F^{-1}\left(\mathrm{t} ; c_{\star}, N(\cdot)\right), \mathrm{C}_{\mathrm{n}}^{\prime}=\mathrm{C}_{\mathrm{n}}^{\prime}\left(\mathrm{C}_{\mathrm{n}}, \mathrm{s}_{0} / c_{\star}\right)$ and $F(\cdot)$ of (1.21),

$$
\sup _{x, y \in V}\left\{\frac{K_{s, t}(x, y)}{\pi_{t}(y)}\right\} \leq e^{2 A} C_{\mathrm{n}}^{\prime} \psi\left(\frac{t-s}{6}\right), \quad \forall s \in[0, t] .
$$

For the dynamic (1.5), replace $\mathcal{N}_{K_{t}^{2}, \pi_{t}}(\mathrm{~s})$ by $2 \mathcal{N}_{K_{t}, \pi_{t}}(\mathrm{~s})$ in (6.2) and $\psi\left(\frac{t-s}{6}\right)$ on the RHS of (6.3) by $\mathbb{E}[\psi(Z)]$, where $Z \sim \frac{1}{3}$ Poisson $(\mathrm{t}-\mathrm{s})$.

(b). If $\left\{a_{t}\right\}$ of (1.31) satisfies (1.30), then for $\mu_{s, t}$ of (1.8), under either (1.3) or (1.5),

$$
\mu_{s, t}(y) \geq e^{-\gamma A} \pi_{t}(y), \quad \forall y \in V, \gamma \in \mathbb{N}, \quad(t+1) \leq 2^{\gamma}(s+1) .
$$

Proof. (a). Fixing $s \in[0, t]$, set $v=(t+s) / 2$, or its integer part in case of DTRW. Note that $t-v \geq(t-s) / 2$ and $(t+1) \leq 4(v+1)$. Further, with $v \in[s, t]$, we have that $K_{s, t}=K_{s, v} K_{v, t}$ and since the Markov kernel $K_{s, v}$ is an $L^{\infty}$-contraction, the LHS of (16.3) is bounded above by $\left\|K_{v, t}\right\|_{L^{1}\left(\pi_{t}\right) \rightarrow L^{\infty}\left(\pi_{v}\right)}$. To bound the latter quantity, consider for $u \geq v$ the non-decreasing $u \mapsto$ $\widehat{\pi}_{u ; v}(\cdot)$. Since $\underline{\widehat{\pi}_{u ; v}} \geq \underline{\pi_{u}} \geq c_{\star}$ and

$$
\mathcal{N}_{K_{u}^{2}, \widehat{\pi}_{u ; v}}(\mathrm{~s})=\mathcal{N}_{K_{u}^{2}, \pi_{u}}\left(e^{a_{v}-a_{u}} \mathrm{~s}\right) \leq N(\mathrm{~s}),
$$

applying Theorem [1.4(a) for the dynamics (1.3) and $\left\{\left(K_{u}, \widehat{\pi}_{u ; v}\right), u \in[v, t]\right\}$, we have that

$$
\left\|K_{v, t}\right\|_{L^{1}\left(\pi_{t}\right) \rightarrow L^{\infty}\left(\pi_{v}\right)}=e^{a_{t}-a_{v}}\left\|K_{v, t}\right\|_{L^{1}\left(\widehat{\pi}_{t ; v}\right) \rightarrow L^{\infty}\left(\widehat{\pi}_{v ; v}\right)} \leq e^{2 A} C_{\mathrm{n}}^{\prime} \psi\left(\frac{t-v}{3}\right)
$$

yielding (6.3). Replacing Theorem 1.4(a) by Theorem 1.4(b), the analogous argument applies for the dynamic (1.5).

(b). Fixing $x_{s}=x$ and $x_{t}=y$, we have from (1.1) that for the dynamic (1.3),

$$
K_{s, t}(x, y)=\sum_{\left\{x_{s+1}, \ldots, x_{t-1}\right\}} \prod_{r=s+1}^{t} \frac{\pi_{r}\left(x_{r}\right)}{\pi_{r}\left(x_{r-1}\right)} K_{r}\left(x_{r}, x_{r-1}\right) \geq \eta_{s}(t) \frac{\pi_{t}(y)}{\pi_{s}(x)}\left[K_{t} \cdots K_{s+1}\right](y, x),
$$

where under (1.31),

$$
\eta_{s}(t):=\prod_{r=s}^{t-1} \inf _{z \in V}\left\{\frac{\pi_{r}(z)}{\pi_{r+1}(z)}\right\} \geq \prod_{r=s}^{t-1} e^{-\left(a_{r+1}-a_{r}\right)}=e^{-\left(a_{t}-a_{s}\right)} .
$$

Multiplying (6.6) by $\pi_{s}(x)$ and summing over $x$ we see that $\mu_{s, t}(y) / \pi_{t}(y) \geq e^{-\left(a_{t}-a_{s}\right)}$, which by (1.30) is further bounded below by $e^{-\gamma A}$ whenever $(t+1) \leq 2^{\gamma}(s+1)$ (see (3.16) ). Next, recall Remark 1.9 that $\mu_{s, t}$ of the CSRW is the expected value over $L \sim \operatorname{Poisson}(t-s)$ and jump times 
$s=T_{0}^{\prime}<T_{1}^{\prime}<\cdots<T_{L}^{\prime} \leq t$ of the value $\mu_{0, L}^{(\omega)}$ for the DTRW using $\left\{\left(K_{T_{m}^{\prime}}, \pi_{T_{m}^{\prime}}\right)\right\}$. Since $a_{T_{m+1}^{\prime}}-a_{T_{m}^{\prime}} \geq$ $\rho_{\boldsymbol{\pi}}\left(T_{m+1}^{\prime}, T_{m}^{\prime}\right)$ for all $m \in \mathbb{N}$, due to (1.31), by the preceding argument, for each $\omega$,

$$
\mu_{0, L}^{(\omega)}(y) \geq e^{-\left(a_{T_{L}^{\prime}}-a_{T_{0}^{\prime}}\right)} \pi_{T_{L}^{\prime}}(y) \geq e^{-\left(a_{t}-a_{s}\right)} \pi_{t}(y) .
$$

Thus, having (1.30) for $t \mapsto a_{t}$, implies that (6.4) holds also for the dynamic (1.5).

Proof of Proposition 1.11. (a) Recall from the proof of Lemma 2.8 that for CSRW or uniformly lazy DTRW on $\boldsymbol{G}_{t}$, the assumed uniform Poincaré inequality and volume growth $v(r)$ with $v(r)$ doubling yield the Nash profile bound for $N(\cdot)$ of (2.21). Following the rest of the proof of Lemma 2.8, while applying Lemma 6.1(a) instead of Theorem 1.4, we deduce that the on-diagonal GHKU bound (2.20) also holds here, for some $C^{\prime}\left(A, \mathrm{C}_{\mathrm{P}}, \mathrm{C}_{\mathrm{v}}, \alpha_{l}\right)$ finite. Next, adapting the proof of Proposition 3.3 we proceed to deduce for $T \geq 1$ the $1 \rightarrow \infty$ norm bound similar to (3.25) for the operator $K_{0,2 T}^{\theta}$. To this end, we use here the dual $\widehat{K}_{s, t}^{\star}$ of $K_{s, t}: L^{2}\left(\widehat{\pi}_{s ; T}\right) \rightarrow L^{2}\left(\widehat{\pi}_{t ; T}\right)$ for the re-scaled non-decreasing conductances $\widehat{\pi}_{r ; T}$ of (6.1), and $r \geq T$. Replacing (3.20), we have

$$
\left\|K_{T, 2 T}^{\theta}\right\|_{L^{1}\left(\pi_{2 T}\right) \rightarrow L^{2}\left(\pi_{T}\right)}=e^{a_{2 T}-a_{T}}\left\|K_{T, 2 T}^{\theta}\right\|_{L^{1}\left(\widehat{\pi}_{2 T ; T}\right) \rightarrow L^{2}\left(\widehat{\pi}_{T ; T}\right)} \leq e^{A}\left\|\left(\widehat{K}_{T, 2 T}^{\star}\right)^{-\theta}\right\|_{L^{2}\left(\widehat{\pi}_{T ; T}\right) \rightarrow L^{\infty}\left(\widehat{\pi}_{2 T ; T}\right)}
$$

so it suffices to bound via Proposition 3.1, the $L^{2}\left(\widehat{\pi}_{T ; T}\right) \rightarrow L^{\infty}\left(\widehat{\pi}_{2 T ; T}\right)$ norm of $\left(\widehat{K}_{T, 2 T}^{\star}\right)^{-\theta}$ as in (3.24) and the $L^{2}\left(\pi_{T}\right) \rightarrow L^{\infty}\left(\pi_{0}\right)$ norm of $K_{0, T}^{\theta}$ as in (3.19). For the $2 \rightarrow \infty$ bound on $\left(\widehat{K}_{T, 2 T}^{\star}\right)^{-\theta}$, recall (3.23) that since $r \mapsto \widehat{\pi}_{r ; T}$ are non-decreasing, $\left\|\widehat{K}_{s, t}^{\star}\right\|_{L^{\infty}\left(\widehat{\pi}_{s ; T}\right) \rightarrow L^{\infty}\left(\widehat{\pi}_{t ; T}\right)} \leq 1$ and Lemma 3.5 provides the $2 \rightarrow 2$ analog of (3.22) for $\left(\widehat{K}_{s, t}^{\star}\right)^{-\theta}$. Further, the $1 \rightarrow \infty$ bound of (2.20) on $K_{s, t}$ yields the $2 \rightarrow \infty$ bound (3.5) with $\varphi(\tau)=C^{\prime} v(\sqrt{\tau})^{-1 / 2}$ and in conjunction with (3.21) for $\widehat{\pi}_{r ; T}$, allows us to apply Proposition 3.1 with this choice of $\varphi(\tau)$ for the adjoint operators $\widehat{K}_{s, t}^{\star}$. Similarly to (3.24) it yields that for some $C_{2}(A)$ and $C_{1}$ finite,

$$
\left\|\left(\widehat{K}_{T, 2 T}^{\star}\right)^{-\theta}\right\|_{L^{2}\left(\widehat{\pi}_{T ; T}\right) \rightarrow L^{\infty}\left(\widehat{\pi}_{2 T ; T}\right)} \leq C_{2} v(\sqrt{T})^{-1 / 2} \exp \left(C_{1} \chi(\theta) T\right) .
$$

Turning next to the $2 \rightarrow \infty$ bound on $K_{0, T}^{\theta}$, utilizing the non-decreasing measures $u \mapsto \widehat{\pi}_{u ; v}$ Lemma 3.5 provides the $2 \rightarrow 2$ bound on $K_{v, u}^{\theta}$ with $\widehat{\pi}_{u ; v}$ replacing $\pi_{u}$ (and $a_{u} \equiv 0$ ). Thus,

$$
\left\|K_{v, u}^{\theta}\right\|_{L^{2}\left(\pi_{u}\right) \rightarrow L^{2}\left(\pi_{v}\right)}=e^{\left(a_{u}-a_{v}\right) / 2}\left\|K_{v, u}^{\theta}\right\|_{L^{2}\left(\widehat{\pi}_{u ; v}\right) \rightarrow L^{2}\left(\widehat{\pi}_{v ; v}\right)} \leq \exp \left(a_{u}-a_{v}+\chi(\theta)(u-v)\right) .
$$

The non-decreasing $t \mapsto a_{t}$ satisfies (1.30), so Proposition 3.1 establishes the bound (3.19) which in turn yields the heat kernel upper bound (1.27) (see our proof of Theorem 1.8(a)).

(b). As in the proof of Theorem (1.8) (b) it suffices to establish the relevant GHKL for $K_{0,2 T}$. To this end, thanks to (6.4) and the assumed uniform volume growth with $v(\cdot)$ doubling, we have that $\left\{\mu_{s, t}(\cdot): s, t \in[T, 2 T]\right\}$ are $c$-stable with respect to $\pi(x) \equiv 1$ (for $c=c\left(\mathrm{C}_{\mathrm{v}}, A\right)$ and all $T$ ). Moreover, in part (a) we established the bound (1.27), therefore $\left\{K_{s, s+t}: s, s+t \in[T, 2 T]\right\}$ satisfy the improved GHKU of (5.17). The PHI is invariant to the re-scaling (6.1) and $a_{t} \leq a_{T}+A$ for $t \in[T, 2 T]$. Hence, considering Theorem 1.6 for $\left(K_{t}, \widehat{\pi}_{t ; T}\right)$, yields for the CSRW that $\left(K_{t}, \pi_{t}\right)$ satisfy the PHI on $[T, 2 T]$, while for DTRW we assumed such PHI, as well as uniformly elliptic conductances. Proceeding as in Step II of the proof of Theorem 1.8(b), yields the GHKL of (1.28) for some $\mathrm{C}_{\star}=\mathrm{C}_{\star}\left(\mathrm{C}_{\mathrm{P}}, \gamma, \mathrm{C}_{\mathrm{v}}, \bar{\alpha}, A\right)$ finite and $K_{T, 2 T}$ (omitting WLOG the bounded factor $\left.\mu_{T, 2 T}\right)$. From the 
upper bound (1.27) it further follows, as in (5.4), that for some $\kappa\left(\mathrm{C}_{\mathrm{P}}, \mathrm{C}_{\mathrm{v}}, \alpha_{l}, A\right)$ finite,

$$
\sum_{z \in B(x, \kappa \sqrt{T})} K_{0, T}(x, z) \geq \frac{1}{2}, \quad \forall x \in V, \quad T \geq 1 .
$$

With $d(z, y)^{2} \leq 2 d(x, z)^{2}+2 d(x, y)^{2}$, we get by combining (1.28) and (6.7), that

$$
K_{0,2 T}(x, y) \geq \frac{1}{2} \inf _{z \in \mathbb{B}(x, \kappa \sqrt{T})}\left\{K_{T, 2 T}(z, y)\right\} \geq \frac{e^{-2 C_{\star} \kappa^{2}}}{2 C_{\star} v(\sqrt{T})} e^{-2 C_{\star} d(x, y)^{2} / T},
$$

whenever $d(x, y) \leq T-\kappa \sqrt{T}$. With $\boldsymbol{G}_{t}$ uniformly elliptic, increasing $\mathrm{C}_{\star}$ (in terms of $\left.\alpha_{e}\right)$, such GHKL extends to all $d(x, y) \in[T-\kappa \sqrt{T}, 2 T]$ (as we have seen already after (5.201) ).

Proof of Proposition 1.13, We refine the counter-example provided in [HK, Proposition 1.4] for $\mathbb{G}=\mathbb{Z}$, by fixing $\eta, \delta_{n} \in(0,1 / 2), \delta_{0}=0$ and setting the uniformly bounded

$$
\pi_{n}(x, x+1)=1+(-1)^{n+x} \eta, \quad \pi_{n}(x, x)=1-(-1)^{n+x} \delta_{n} .
$$

Then, $\pi_{n}(x)=3-(-1)^{n+x} \delta_{n}$ satisfy (1.31) with $a_{n+1}-a_{n} \leq \frac{2}{5}\left(\delta_{n}+\delta_{n+1}\right)$, and $K_{n}(x, y)$ of (1.1) satisfies (1.12)-(1.13) with $\bar{\alpha}=1 / 7$. The process $\left\{X_{n}\right\}$ induces on types $A$ and $B$ that correspond to $n+X_{n}$ being even and odd, respectively, the in-homogeneous $\{A, B\}$-valued Markov chain of transition probabilities:

$$
\begin{aligned}
& q_{n}(A, B)=\frac{1-\delta_{n}}{3-\delta_{n}}, \quad q_{n}(A, A)=\frac{2}{3-\delta_{n}}, \\
& q_{n}(B, A)=\frac{1+\delta_{n}}{3+\delta_{n}}, \quad q_{n}(B, B)=\frac{2}{3+\delta_{n}} .
\end{aligned}
$$

The uniformly bounded increments $X_{n+1}-X_{n}$ are zero on transitions between types $A$ and $B$ and otherwise they are \pm 1 -valued of mean $\Delta_{n}(A)=\frac{2 \eta}{3-\delta_{n}}$ and $\Delta_{n}(B)=-\frac{2 \eta}{3+\delta_{n}}$, when at time $n$ the type is $A$ or $B$, respectively. Note that

$$
v_{n}:=\frac{1}{2} \sum_{i=1}^{n}\left(\Delta_{i}(A)+\Delta_{i}(B)\right) \geq \frac{2 \eta}{9} a_{n}=O\left(n^{1 / 2+\iota}\right),
$$

and starting at $X_{0}=0$ (i.e. at type $A$ ), since $q_{n}(B, A)>1 / 3>q_{n}(A, B)$ for all $n$, such $\{A, B\}$ valued Markov chain induces the drift $\mathbb{E} X_{n} \geq v_{n}$. Thus, from the concentration of the pair-empirical $\{A, B\}$-valued measure around its limit $[2 / 6,1 / 6,1 / 6,2 / 6]$, we deduce that for some $C>0$,

$$
p_{n}:=\mathbb{P}\left(\left|X_{n}\right| \leq C n^{(1+\iota) / 2}\right) \leq C^{-1} \exp \left(-C n^{\iota}\right) .
$$

It is easy to check that if the lower bound of (1.7) held for the uniformly bounded above and below $\pi_{n}(\cdot)$, then necessarily $\inf _{n}\left\{p_{n}\right\}>0$ in contradiction with (6.8). Further, even if only the upper bound of (1.6) held for $\pi_{n}(\cdot)$, then since $\iota>0$ necessarily $\mathbb{P}\left(\left|X_{n}\right|>C n^{(1+\iota) / 2}\right) \rightarrow 0$, again contradicting (6.8).

Acknowledgments. We thank Takashi Kumagai for proposing to pursue the GHK in our aim to verify ABGK, Conj. 7.1]. This work further benefited from discussions (of A.D.) with Martin Barlow and (of T.Z.) with Laurent Saloff-Coste. 


\section{REFERENCES}

[ABGK] G. Amir, I. Benjamini, O. Gurel-Gurevich and G. Kozma. Random walk in changing environment. ArXiv:1504.04870v3 (2017).

[Ba] M.T. Barlow. Random walks on supercritical percolation clusters. Ann. Probab. 32 (2004), 3024-3084.

[Ba2] M.T. Barlow. Random walks and heat kernels on graphs. London mathematical society lecture notes series 438 , Cambridge University Press, 2017.

[BC] M.T. Barlow and X. Chen. Gaussian bounds and parabolic Harnack inequality on locally irregular graphs. Math. Ann. 366 (2016), 1677-1720.

[BPS] A. Bendikov, C. Pittet and R. Sauer. Spectral distribution and $L^{2}$-isoperimetric profile of Laplace operators on groups. Math. Ann. 354 (2012), 43-72.

[MB] M. Biskup. Recent progress on the random conductance model. Prob. Surveys 8 (2011) $294-373$.

[BK] R.F. Bass and T. Kumagai. Symmetric Markov chains on $\mathbb{Z}^{d}$ with unbounded jumps. Trans. Amer. Math. Soc. 360 (2008), 2041-2075.

[C1] T. Coulhon. Dimensions at infinity for Riemannian manifolds. Potential Anal. 4 (1995), 335-344.

[C2] T. Coulhon. Espaces de Lipschitz et inégalités de Poincaré. J. Funct. Anal. 136 (1996), 81-113.

[C3] T. Coulhon. Ultracontractivity and Nash type inequalities. J. Funct. Anal. 141 (1996), 510-539.

[CG] T. Coulhon and A. Grigor'yan. Random walk on graphs with regular volume growth. Geom. Funct. Anal. 8 (1998), 656-701.

[CGZ] T. Coulhon, A. Grigor'yan and F. Zucca. The discrete integral maximum principle and its applications. Tohoku Math. J. 57 (2005), 559-587.

[CK] Z.-Q. Chen and T. Kumagai, Heat kernel estimates for jump processes of mixed types on metric measure spaces. Probab. Theory Relat. Fields. 140 (2008), 277-317.

[Del] T. Delmotte. Parabolic Harnack inequality and estimates of Markov chains on graphs. Rev. Mat. Iberoam. 15, (1999), 181-232.

[DD] T. Delmotte and J.-D. Deuschel. On estimating the derivatives of symmetric diffusions in stationary random environment, with applications to $\nabla \phi$ interface model. Probab. Theory Relat. Fields 133 (2005), 358-390.

[DHMP] A. Dembo, R. Huang, B. Morris and Y. Peres. Transience in growing subgraphs via evolving sets. Ann. Inst. H. Poincaré Probab. Stat. 53 (2017), 1164-1180.

[Fo] M. Folz. Gaussian upper bounds for heat kernels of continuous time simple random walks. Elect. J. Probab. 16 (2011), 1693-1722.

[FS] E.B. Fabes and D.W. Stroock. A new proof of Moser's parabolic Harnack inequality via the old ideas of Nash. Arch. Rat. Mech. Anal. 96 (1986), 327-338.

[GMT] S. Goel, R. Montenegro and P. Tetali. Mixing time bounds via the spectral profile. Elect. J. Probab. 11 (2006), $1-26$.

[Gr] A. Grigor'yan. The heat equation on noncompact Riemannian manifolds. (in Russian) Matem. Sbornik. 182 (1991), 55-87. (English transl.) Math. USSR Sbornik 72 (1992), 47-77.

[GOS] G. Giacomin, S. Olla and H. Spohn. Equilibrium fluctuation for $\nabla \varphi$ interface model. Ann. Probab. 29 (2001), 1138-1172.

[HK] R. Huang and T. Kumagai. Stability and instability of Gaussian heat kernel estimates for random walks among time-dependent conductances. Elect. Comm. Probab. 21 (2016), no. 5, 1-11.

[HS] W. Hebisch and L. Saloff-Coste. Gaussian estimates for Markov chains and random walks on groups. Ann. Probab. 21 (1993), 673-709.

[HS2] W. Hebisch and L. Saloff-Coste. On the relation between elliptic and parabolic Harnack inequalities. Ann. Inst. Fourier (Grenoble). 51 (2001), no.5, 1437-1481.

[Kum] T. Kumagai. Random walks on disordered media and their scaling limits, Lect. Notes in Math. 2101, Ecole d'ete de probabilites de Saint-Flour XL-2010, Springer, New York 2014.

[LS] G. F. Lawler and A. D. Sokal. Bounds on the $L^{2}$ spectrum for Markov chains and Markov processes: a generalization of Cheeger's inequality. Trans. Amer. Math. Soc. 309 (1988), 557-580.

[MO] J.-C. Mourrat and F. Otto. Anchored Nash inequalities and heat kernel bounds for static and dynamic degenerate environments. J. Funct. Anal. 270 (2016), 201-228.

[SC] L. Saloff-Coste. A note on Poincaré, Sobolev, and Harnack inequalities. Inter. Math. Res. Notices 2 (1992), 27-38.

[SC2] L. Saloff-Coste. Aspects of Sobolev-type inequalities. London mathematical society lecture notes series 289, Cambridge University Press, 2002. 
[SZ1] L. Saloff-Coste and J. Zúñiga. Merging for inhomogeneous finite Markov chains, part I: singular values and stability. Elect. J. Probab. 14 (2009), 1456-1494.

[SZ2] L. Saloff-Coste and J. Zúñiga. Merging for inhomogeneous finite Markov chains, part II: Nash and log-Sobolev inequalities. Ann. Probab. 39 (2011), 1161-1203.

[SZ3] L. Saloff-Coste and J. Zúñiga. Merging and stability for time inhomogeneous finite Markov chains. Surveys in stochastic processes, 127-151, Eur. Math. Soc. (2011).

[SZh] D.W. Stroock and W. Zheng. Markov chain approximations to symmetric diffusions. Ann. Inst. Henri Poincaré (B) 33 (1997), 619-649.

[St1] K.T. Sturm. Analysis on local Dirichlet spaces II. Upper Gaussian estimates for the fundamental solutions of parabolic equations. Osaka J. Math. 32 (1995), 275-312.

[St2] K.T. Sturm. Analysis on local Dirichlet spaces III. The parabolic Harnack inequality. J. Math. Pures Appl. 75 (1996), 273-297.

[SW] E.M. Stein, and G.L. Weiss. Introduction to Fourier analysis on Euclidean spaces. Vol. 1. Princeton university press, 1971. USA

Department of Mathematics, Stanford University. Building 380, Sloan Hall, Stanford, CA 94305 ,

Department of Statistics, Stanford University. Sequoia Hall, 390 Serra Mall, Stanford, CA 94305 , USA.

Department of Mathematics, University of California, San Diego. 9500 Gilman Dr, La Jolla, CA 92093, USA. 